\title{
Low-frequency Faraday rotation measures towards pulsars using LOFAR: probing the 3-D Galactic halo magnetic field
}

DOI:

10.1093/mnras/stz214

\section{Document Version}

Accepted author manuscript

Link to publication record in Manchester Research Explorer

\section{Citation for published version (APA):}

Sobey, C., Bilous, A. V., Griessmeier, J., Hessels, J. W. T., Karastergiou, A., Keane, E. F., Kondratiev, V. I., Kramer, M., Michilli, D., Noutsos, A., Pilia, M., Polzin, E., Stappers, B., Tan, C. M., van Leeuwen, J., Verbiest, J. P. W., Weltevrede, P., Heald, G., Alves, M. I. R., ... Van Eck, C. (2019). Low-frequency Faraday rotation measures towards pulsars using LOFAR: probing the 3-D Galactic halo magnetic field. Monthly Notices of the Royal Astronomical Society. https://doi.org/10.1093/mnras/stz214

\section{Published in:}

Monthly Notices of the Royal Astronomical Society

\section{Citing this paper}

Please note that where the full-text provided on Manchester Research Explorer is the Author Accepted Manuscript or Proof version this may differ from the final Published version. If citing, it is advised that you check and use the publisher's definitive version.

\section{General rights}

Copyright and moral rights for the publications made accessible in the Research Explorer are retained by the authors and/or other copyright owners and it is a condition of accessing publications that users recognise and abide by the legal requirements associated with these rights.

\section{Takedown policy}

If you believe that this document breaches copyright please refer to the University of Manchester's Takedown Procedures [http://man.ac.uk/04Y6Bo] or contact uml.scholarlycommunications@manchester.ac.uk providing relevant details, so we can investigate your claim.

\section{OPEN ACCESS}




\title{
Low-frequency Faraday rotation measures towards pulsars using LOFAR: probing the 3-D Galactic halo magnetic field
}

\author{
C. Sobey ${ }^{1,2,3 \star}$, A. V. Bilous ${ }^{4}$, J-M. Grießmeier ${ }^{5,6}$, J. W. T. Hessels ${ }^{3,4}$,
}

A. Karastergiou ${ }^{7,8,9}$, E. F. Keane ${ }^{10}$, V. I. Kondratiev ${ }^{3,11}$, M. Kramer ${ }^{12}$, D. Michilli ${ }^{4,3,13,14}$, A. Noutsos ${ }^{12}$, M. Pilia ${ }^{15}$, E. J. Polzin ${ }^{16}$, B. W. Stappers ${ }^{16}$, C. M. Tan ${ }^{16}$,

J. van Leeuwen ${ }^{3}$, J. P. W. Verbiest ${ }^{17,12}$, P. Weltevrede ${ }^{16}$, G. Heald ${ }^{2}$, M. I. R. Alves ${ }^{18}$, E. Carretti ${ }^{19}$, T. Enßlin ${ }^{20}$, M. Haverkorn ${ }^{18}$, M. Iacobelli ${ }^{3}$, W. Reich ${ }^{12}$, C. Van Eck $^{21}$

${ }^{1}$ International Centre for Radio Astronomy Research - Curtin University, GPO Box U1987, Perth, WA 6845, Australia

${ }^{2}$ CSIRO Astronomy and Space Science, PO Box 1130 Bentley, WA 6102, Australia

${ }^{3}$ ASTRON, Netherlands Institute for Radio Astronomy, Oude Hoogeveensedijk 4, 7991 PD, Dwingeloo, The Netherlands

${ }^{4}$ Anton Pannekoek Institute for Astronomy, University of Amsterdam, Science Park 904, 1098 XH, Amsterdam, The Netherlands

${ }^{5}$ LPC2E - Université d'Orléans / CNRS, 45071 Orléans cedex 2, France

${ }^{6}$ Station de Radioastronomie de Nançay, Observatoire de Paris, PSL Research University, CNRS, Univ. Orléans, OSUC, 18330 Nançay, France

${ }^{7}$ Oxford Astrophysics, Denys Wilkinson Building, Keble Road, Oxford OX1 3RH, UK

${ }^{8}$ Department of Physics \& Astronomy, University of the Western Cape, Private Bag X17, Bellville 7535, South Africa

${ }^{9}$ Department of Physics and Electronics, Rhodes University, PO Box 94, Grahamstown 6140, South Africa

${ }^{10}$ SKA Organisation, Jodrell Bank Observatory, SK11 9DL, UK

${ }^{11}$ Astro Space Centre, Lebedev Physical Institute, Russian Academy of Sciences, Profsoyuznaya Str. 84/32, Moscow 117997, Russia

${ }^{12}$ Max Planck Institute for Radio Astronomy, Auf dem Hügel 69, 53121 Bonn, Germany

${ }^{13}$ Department of Physics, Mc Gill University, 3600 University Street, Montréal, QC H3A 2T8, Canada

${ }^{14}$ Mc Gill Space Institute, McGill University, 3550 University Street, Montréal, QC H3A 2A7, Canada

${ }^{15}$ INAF - Osservatorio Astronomico di Cagliari, via della Scienza 5, I-09047 Selargius (CA), Italy

${ }^{16}$ Jodrell Bank Centre for Astrophysics, School of Physics and Astronomy, The University of Manchester, Manchester, M13 9PL, UK

${ }^{17}$ Fakultät für Physik, Universität Bielefeld, Postfach 100131, 33501 Bielefeld, Germany

${ }^{18}$ Department of Astrophysics/IMAPP, Radboud University Nijmegen, PO Box 9010, 6500 GL Nijmegen, The Netherlands

${ }^{19}$ INAF - Istituto di Radioastronomia, Via Gobetti 101, 40129 Bologna, Italy

${ }^{20}$ Max Planck Institut für Astrophysik, Karl-Schwarzschild-Str 1, D-85740 Garching, Germany

${ }^{21}$ Dunlap Institute for Astronomy and Astrophysics, University of Toronto, 50 St. George Street, Toronto, ON M5S 3H4, Canada

\begin{abstract}
We determined Faraday rotation measures (RMs) towards 137 pulsars in the northern sky, using Low-Frequency Array (LOFAR) observations at 110-190 MHz. This low-frequency RM catalogue, the largest to date, improves the precision of existing RM measurements on average by a factor of 20 - due to the low frequency and wide bandwidth of the data, aided by the RM synthesis method. We report RMs towards 25 pulsars for the first time. The RMs were corrected for ionospheric Faraday rotation to increase the accuracy of our catalogue to $\approx 0.1 \mathrm{rad} \mathrm{m}{ }^{-2}$. The ionospheric RM correction is currently the largest contributor to the measurement uncertainty. In addition, we find that the Faraday dispersion functions towards pulsars are extremely Faraday thin - mostly less than $0.001 \mathrm{rad} \mathrm{m}^{-2}$. We use these new precise RM measurements (in combination with existing RMs, dispersion measures, and distance estimates) to estimate the scale height of the Galactic halo magnetic field: $2.0 \pm 0.3 \mathrm{kpc}$ for Galactic quadrants I and II above and below the Galactic plane (we also evaluate the scale height for these regions individually). Overall, our initial low-frequency catalogue provides valuable information about the 3-D structure of the Galactic magnetic field.
\end{abstract}

Key words: pulsars: general - techniques: polarimetric - radio telescopes - ISM: magnetic fields - Galaxy: structure 


\section{INTRODUCTION}

Magnetic fields are ubiquitous in the Universe. They play a role in numerous astrophysical processes across a range of physical scales and field strengths. Within galaxies, magnetic fields pervade the multi-phase diffuse interstellar medium (ISM).

Galactic magnetic fields can be modelled as a combination of a large, kiloparsec-scale, coherent component; a component with a power spectrum of small-scale (1-100 parsec), random fluctuations; and an intermediate-scale component that is ordered overall but with small-scale field direction reversals (e.g. Jaffe et al. 2010). The coherent component observed, for example, in 'magnetic spiral arms' (e.g. Beck 2009) accelerates and confines cosmic rays (e.g. Aharonian et al. 2012, and references therein) and may be maintained by a turbulent dynamo (e.g. Dobbs et al. 2016). The random component, caused by turbulence, supernovae and shocks, and other localised phenomena (e.g. Haverkorn et al. 2015) plays a role in, for example, the formation of molecular clouds and stars (e.g. Crutcher 2012). The intermediate ordered component is thought to result from larger-scale effects, including shearing dynamics and compression, on the random component (e.g. Jaffe et al. 2010) and plays a role in, for example, the transport of heat and angular momentum (e.g. Haverkorn 2015). In the Milky Way, the random and ordered components can be between 0.5 - and 5-times the magnitude of the coherent component, depending on the observables (see below), regions studied, and assumptions used (e.g. Rand \& Kulkarni 1989; Haverkorn et al. 2004, 2006; Schnitzeler et al. 2007; Jaffe et al. 2010, 2011; Beck et al. 2016).

The (magnetic) structure of our Galaxy is challenging to study because we are embedded within it. The Galactic magnetic field (GMF) was first measured over 65 years ago using polarisation of starlight (Hall 1949; Hiltner 1949). There are several other observables that provide information about the GMF, including Zeeman splitting of spectral lines (e.g. Crutcher et al. 2010); polarised thermal emission from dust grains (e.g. Planck Collaboration et al. 2016); total intensity and polarisation of Galactic synchrotron emission (e.g. Sun et al. 2008); Faraday rotation of polarised sources (e.g. Van Eck et al. 2011); and ultra-high energy cosmic rays (e.g. Farrar et al. 2013). These observables (mostly 2-D tracers requiring ancillary data) usually provide information about the strength/direction of at least one component of the magnetic field parallel/perpendicular to the line-of-sight ( $\mathrm{LoS})$ within a particular phase of the ISM (e.g. Ferrière 2011; Haverkorn 2015). Several of these observables will be required to accurately reconstruct the multiple-scale components of the GMF (e.g. Jaffe et al. 2010; Boulanger et al. 2018).

Our current picture of the large-scale GMF is that the field strength is on the order of $\sim 2-10 \mu \mathrm{G} ; \approx 2 \mu \mathrm{G}$ at the solar position and increasing towards the Galactic centre and decreasing towards the anticentre (e.g. Beck 2001; Brown et al. 2003; Han et al. 2006). Recent analyses of the large-scale disc component often favour an axisymmetric spiral with an overall clockwise direction (as viewed from the north Galactic pole) that seems to somewhat follow the spiral-arm structure, with one field direction reversal near the Sun in the direction of the Galactic centre along the Scutum-Centaurus arm (e.g. Van Eck et al. 2011; Jansson \& Farrar 2012). However, the total number and locations of the large-scale GMF reversals are still under debate (e.g. Han et al. 2006), and many open questions concerning the 3-D structure of the aggregate GMF (along with its generation and evolution) remain (e.g. Ferrière 2011). Furthermore, such reversals have not yet been observed in any other galaxies (e.g. Beck et al. 2015). Another component of the large-scale GMF often considered separately to the disc, is the halo (absolute Galactic latitude greater than a few degrees, e.g., Han et al. 2018). The 3D GMF structure in the halo is less well understood, with several proposed geometries (see, e.g., Haverkorn 2015; Terral \& Ferrière 2017, and references therein), including a north-south asymmetry across the Galactic disc (Mao et al. 2012). Despite the challenges, our Galaxy also provides a unique opportunity to study the magnetic field structure at much smaller scales, essential for comparison with nearby galaxies (e.g. Nota \& Katgert 2010).

Faraday rotation measurements of polarised radio sources have been used to measure the magnetic field strength and direction in the intervening (warm) ISM parallel to the LoS. This method has been used to investigate the structure of the GMF by using a large sample of sources, including pulsars (e.g. Manchester 1972, 1974; Rand \& Lyne 1994; Han et al. 1999, 2006; Noutsos et al. 2008), extragalactic sources (e.g. Mao et al. 2010; Oppermann et al. 2012, 2015), and a combination of both (e.g. Brown et al. 2007; Van Eck et al. 2011). Polarisation observations of a large set of pulsars can be used to probe the 3-D structure of the GMF efficiently. The ratio between the Faraday rotation measure (RM) and the dispersion measure (DM) towards a pulsar at distance $d$ (pc) provides an estimate of the electron density-weighted average magnetic field strength and net direction parallel to the LoS:

$$
\left\langle B_{\|}\right\rangle=\frac{\int_{0}^{d} n_{\mathrm{e}} B_{\|} \mathrm{d} l}{\int_{0}^{d} n_{\mathrm{e}} \mathrm{d} l}=1.232 \mu \mathrm{G}\left(\frac{\mathrm{RM}}{\mathrm{rad} \mathrm{m}^{-2}}\right)\left(\frac{\mathrm{DM}}{\mathrm{pc} \mathrm{cm}^{-3}}\right)^{-1},
$$

where $n_{\mathrm{e}}$ is the electron density and $\mathrm{d} l$ is the differential distance element. By definition, positive (negative) RMs indicate that the net direction of $\left\langle B_{\|}\right\rangle$is towards (away from) the observer. Equation 1 assumes that the electron density and magnetic field are uncorrelated (e.g. Beck et al. 2003). Furthermore, pulsar emission is often highly (linearly) polarised (e.g. Johnston \& Kerr 2018), and Faraday rotation internal to the pulsar magnetosphere is negligible (e.g. Wang et al. 2011), facilitating measurement of the RM due to the foreground ISM alone. Thus, it is also expected that pulsars are 'Faraday thin' point sources, where the polarised flux detected lies at a single Faraday depth (i.e. the RM dispersion is negligible; in contrast to a Faraday thick source with polarised flux distributed over a range of Faraday depths). There are 2659 known pulsars ${ }^{1}$ distributed throughout the Galaxy, particularly near the disc (Manchester et al. 2005). Currently, 1133 pulsars (43 per cent) have published RMs - two-thirds of which are located in the Southern sky, near the Galactic plane, and are mostly concentrated within a few kiloparsecs from the Sun (Manchester et al. 2005). The mode $\mathrm{RM}$ measurement in the pulsar catalogue was taken at the Parkes Observatory at a frequency of $\approx 1.4 \mathrm{GHz}$, using $128 \mathrm{MHz}$ bandwidth (e.g. Han et al. 2006; Noutsos et al. 2008; Han et al. 2018). Pulsar distances are estimated using their DMs and a model of the Galactic thermal electron density, e.g., TC93 (Taylor \& Cordes 1993); NE2001 (Cordes \& Lazio 2002); YMW16 (Yao et al. 2017). However, these distance estimates can be quite uncertain, and only $\approx 200$ pulsars have independent distance measurements (e.g. Yao et al. 2017; Deller et al. 2018). Therefore, it is possible that what are interpreted as field reversals along spiral arms may be caused by other intermediate-scale structures, e.g., superbubbles (Haverkorn 2015). RMs measured towards extragalactic sources provide complementary information about the integrated LoS through (at least) the Galaxy, which partially smooths out smaller scale structure.

${ }^{1}$ Current version of pulsar catalogue (1.59) retrieved from http://www.atnf.csiro.au/people/pulsar/psrcat/ 
Work towards better understanding astrophysical magnetic fields, including the GMF, is ongoing. The recent construction of low-frequency $(<300 \mathrm{MHz})$, next-generation aperture array telescopes (and their associated supercomputing facilities) has rejuvenated this field. These include the Low-Frequency Array (LOFAR; van Haarlem et al. 2013) and the Long-Wavelength Array (LWA; Taylor et al. 2012) in the Northern Hemisphere, and the Murchison Widefield Array (MWA; Tingay et al. 2013) in the Southern Hemisphere. Polarisation observations from low-frequency telescopes such as these are providing precise measurements of ISM parameters, due to the wavelength-squared dependencies from the effects of dispersion and Faraday rotation (e.g. Stovall et al. 2015; Kondratiev et al. 2016; Lenc et al. 2016; Van Eck et al. 2017). These facilities are the pathfinders or the precursor to the low-frequency aperture array component of the Square Kilometre Array (SKA-Low), which will operate at $50-350 \mathrm{MHz}$ (Keane 2018). The SKA's capabilities for pulsar discoveries, timing, and astrometry will revolutionise radio astronomy and will be invaluable for studying the GMF in 3-D on large and small scales (e.g. Han et al. 2015).

In this paper, we present RM measurements towards 137 pulsars using low-frequency $(<200 \mathrm{MHz})$ polarisation observations from the 'LOFAR census of non-recycled pulsars' (Bilous et al. 2016) and the 'LOFAR census of millisecond pulsars' (Kondratiev et al. 2016). This also includes RM measurements towards PSR B0329+54, using several LOFAR (timing/monitoring) observations to further investigate the accuracy of the current ionospheric RM correction method. This is the largest low-frequency RM catalogue to date and is also the first to report a large number of RM dispersion measurements for pulsars. These measurements are complementary to similar low-frequency RM catalogues towards extragalactic sources that trace LoSs through (at least) the entire Galaxy (e.g. Neld et al. 2018; Van Eck et al. 2018; Riseley et al. 2018), as well as polarisation observations of diffuse Galactic synchrotron emission that traces the GMF in the plane of the sky (e.g. Jelić et al. 2015; Van Eck et al. 2017). We aim to increase the number of pulsars with RM data and provide higher-precision RM measurements for those already in the literature, to study the large-scale structure of the GMF in the Galactic halo. These data also provide a baseline measurement towards monitoring fluctuations in RM over time to investigate the small-scale magneto-ionic structure in the ISM in the future.

The LOFAR observations and data reduction are described in Section 2. In Section 3 we present a summary of our catalogue of low-frequency DMs and RMs. In Section 4 we discuss our results, including an estimate of the scale height of the Galactic halo magnetic field, and address the limitations of the current data and methods. We provide a summary and our conclusions in Section 5. Appendix A presents the table of LOFAR RM and RM dispersion measurements, and Appendix B presents further details of the RM-synthesis analysis and the Faraday dispersion functions (FDFs or Faraday spectra) obtained for each pulsar with a significant RM detection.

\section{OBSERVATIONS AND DATA REDUCTION}

All of the observations were performed using LOFAR, which can observe the northern sky between $10-90$ and $110-240 \mathrm{MHz}$ (for a description of LOFAR see van Haarlem et al. 2013). The large instantaneous bandwidth available (up to $96 \mathrm{MHz}$ ) and large collecting area (up to 24 core stations in the tied-array mode) provide high-quality polarisation data at low frequencies that are well-suited to measuring precise RMs (e.g. Noutsos et al. 2015). The LOFAR observations of pulsars presented in this paper were conducted between 2012 December and 2014 November, with a typical integration time of $\geqslant 20 \mathrm{~min}$, using the observations summarised in Bilous et al. (2016) and Kondratiev et al. (2016).

The signals received from between 20 and 24 of the LOFAR High-Band-Antenna (HBA) core stations were coherently combined, using the single distributed clock signal and the LOFAR correlator/beam-former, to establish a tied-array beam (for a description of LOFAR's pulsar observing modes see Stappers et al. 2011). The pulsar observations were recorded at a centre frequency of $148.9 \mathrm{MHz}$, with $78.1 \mathrm{MHz}$ of contiguous bandwidth using the eight-bit sampling mode. The data from the observations were recorded in one of two formats. For the millisecond pulsars (MSPs), the raw complex-voltage $(\mathrm{CV})$ data were recorded with a sampling time of $5.12 \mu \mathrm{s}$ and a channel width of $195.3 \mathrm{kHz}$, allowing the data to be coherently dedispersed (e.g. Kondratiev et al. 2016). For the slower non-recycled pulsars with larger rotational periods (allowing larger sampling times and incoherent dedispersion, resulting in smaller data volume), the Stokes $I Q U V$ parameters were computed and recorded with a sampling time (1-8) $\times 163.8 \mu$ s and each $195.3 \mathrm{kHz}$ sub-band was split into an additional $(1-8) \times 32$ channels (e.g. Bilous et al. 2016).

The recorded data were pre-processed using the LOFAR PULsar Pipeline (PULP, a Python-based suite of scripts that provides basic offline pulsar processing; Kondratiev et al. 2016). The dedispersion and folding were performed using each pulsar's timing ephemeris obtained from Jodrell Bank Observatory or the Green Bank Telescope where available (e.g. Pilia et al. 2016; Bilous et al. 2016), or the pulsar catalogue. The dedispersion and folding were performed on each frequency channel using the DSPSR ${ }^{2}$ digital signal-processing software (van Straten \& Bailes 2011) and written as a PSRFITS $^{3}$ archive (Hotan et al. 2004). The folding produced sub-integrations of between $5 \mathrm{~s}$ and $1 \mathrm{~min}$. Radio frequency interference (RFI) in the data was initially removed in affected frequency subbands and time subintegrations using automated programs, i.e., PAZ from the PSRCHIVE ${ }^{4}$ software suite (Hotan et al. 2004), and CLEAN.PY from the COASTGUARD ${ }^{5}$ software suite (Lazarus et al. 2016). The archives were averaged in frequency to 400 channels and stored in the LOFAR Long-Term Archive ${ }^{6}$. The optimal DM and period $(P)$ of the pulsars were determined previously (Bilous et al. 2016; Kondratiev et al. 2016). The data were inspected to excise obvious remaining RFI using the interactive PSRCHIVE PAZI program. Table 1 shows a summary of the observational and corresponding RM-synthesis parameters, see Section 2.1.

The data have not yet been polarisation calibrated, to account for the parallactic angle or the tied-array instrument beam. The polarisation calibration will be implemented in future work, which will also present the average polarisation profiles of the data described here. For aperture arrays, including LOFAR, polarisation calibration is non-trivial. There are no wavelength-squared-dependent variables in LOFAR's beam response model, especially very close to the pointing centre (where all of the observed pulsars were located), and the parallactic angle is independent of frequency (e.g. Noutsos et al. 2015), leaving the Faraday rotation signal in the data unaf-

\footnotetext{
2 http://dspsr.sourceforge.net

3 http://www.atnf.csiro.au/research/pulsar/psrfits/

4 http://psrchive.sourceforge.net

5 https://github.com/plazar/coast_guard

6 https://lta.lofar.eu/
} 
fected. Using observations of nine of the pulsars in this work, we verified that the RMs determined before the current polarisation calibration pipeline are equal to those post-calibration. Moreover, the low-frequency data from LOFAR provide a wide bandwidth, throughout which even low RM values cause the Stokes $Q, U$ parameters to vary over many sinusoidal cycles. For example, a small $\mathrm{RM}$ of just $1 \mathrm{rad} \mathrm{m}^{-2}$ causes the Stokes $Q, U$ parameters to undergo over 1.5 sinusoidal cycles across the $110-190 \mathrm{MHz}$ recorded bandwidth range. Therefore, the Faraday rotation effect that causes the sinusoidal variation in the Stokes $Q, U$ signal with wavelength squared (see Section 2.1) is distinct compared to other possible wavelength-dependant variations due to, e.g., telescope beam effects or pulse profile evolution. LOFAR's X-Y dipoles are rotated 45 degrees away from North, so that when the Stokes parameters are recorded, the RM sign is opposite to the IAU convention (Heald et al. 2018). Therefore, the signs of the RMs measured in this work were flipped to be consistent with the IAU convention used in the pulsar catalogue. The LOFAR beam model corrects this effect, so this sign flip will not be necessary post-polarisation calibration.

Table A1 provides the additional observational parameters (i.e. Modified Julian Date) for each pulsar with a measured RM.

\subsection{Determining RMs using RM-synthesis}

A polarised wave travelling through a magnetised plasma (such as the ISM) is subject to the effect of Faraday rotation: the polarisation angle, $\chi=0.5 \tan ^{-1}(U / Q)(\mathrm{rad})$, rotates as a function of the wavelength, $\lambda(\mathrm{m})$, squared:

$\chi\left(\lambda^{2}\right)=\chi_{0}+\mathrm{RM} \lambda^{2}$,

where the $\mathrm{RM}\left(\mathrm{rad} \mathrm{m}^{-2}\right)$ characterises the magnitude of the effect, and is dependent on the integrated electron density and magnetic field strength parallel to the LoS, see Equation 1. $\chi_{0}$ represents the intrinsic polarisation angle of the polarised source's emission.

It has been common practice to measure RMs by determining the gradient of the observed polarisation angle as a function of wavelength squared (e.g. Rand \& Lyne 1994). More recently, advancements in computing power have facilitated the recording of larger bandwidths from radio observations and have enabled the use of the powerful method of RM-synthesis (Burn 1966; Brentjens \& de Bruyn 2005) for investigating magnetic fields (e.g. Heald et al. 2009; Lenc et al. 2016; Van Eck et al. 2017). This method takes advantage of the Fourier-like relationship between the complex polarisation intensity vector as a function of wavelength squared, $P\left(\lambda^{2}\right)=Q\left(\lambda^{2}\right)+\mathrm{i} U\left(\lambda^{2}\right)$, and the Faraday dispersion function (FDF; also referred to as the Faraday spectrum, $F(\phi))$ as a function of Faraday depth, $\phi$, advantageously using the entire frequency coverage of the data simultaneously for determining the Faraday depth or RM. Following Brentjens \& de Bruyn (2005), the FDF is given by:

$\tilde{F}(\phi) \approx K \sum_{i=1}^{\mathrm{N}} \tilde{P}\left(\lambda_{i}^{2}\right) e^{-2 i \phi\left(\lambda_{i}^{2}-\lambda_{0}^{2}\right)}$,

where $\lambda_{i}$ is the central wavelength of channel $i$ from the observation; $\lambda_{0}$ is the reference wavelength (ideally the weighted average of $\lambda_{i}^{2}$ ); $\tilde{P}\left(\lambda_{i}^{2}\right)=w_{i} P\left(\lambda_{i}^{2}\right)=w_{i}\left[Q\left(\lambda_{i}^{2}\right)+\mathrm{i} U\left(\lambda_{i}^{2}\right)\right]$; and $K$ is the inverse sum of all weights $w_{i}$. We allowed the weights $w_{i}$ to be set to an equal value for all observed wavelengths. In the context of this work, $\mathrm{RM}$ and $\phi$ terms can be used interchangeably. Here, we use ' $\phi$ ' in reference to the FDF outputs from RM-synthesis, and 'RM' in reference to the measurements obtained from the FDFs (as well as from the pulsar and extragalactic catalogues). See Figure 1 for the
RM spread function (RMSF; analogous to an optical telescope's point spread function, formally RMTF in Brentjens \& de Bruyn 2005), showing the theoretical response of the wavelength squared coverage of our LOFAR data in Faraday depth.

For each average LOFAR pulsar profile, the on-pulse Stokes parameters (for each frequency channel) were extracted using the PSRCHIVE RMFIT routine. For profiles with significant signal-tonoise in linear polarisation (where $\mathrm{S} / \mathrm{N}_{P}>7$ ), the selected on-pulse region was identified as the phase bin with the largest $\mathrm{S} / \mathrm{N}_{P}$. Since the profiles were not yet corrected for Faraday rotation, many of the linear polarisation profiles were depolarised, and in these cases $\left(\mathrm{S} / \mathrm{N}_{P} \leqslant 7\right)$ the pulse phase bin with the highest total intensity (Stokes $I$ ) was selected as the on-pulse phase bin. We used a small number of on-pulse pulse profile bins to extract the Stokes parameters because this seems to minimise the instrumental polarisation fraction in the data, see below and Appendix B for further discussion.

The Stokes-parameter data were used as the input to a publicly available RM-synthesis program ${ }^{7}$, written in PYTHON, which computed the FDF. An example FDF obtained from a LOFAR observation of PSR B0329+54 is shown in Figure 2. The FDFs were computed in the range $-330 \leqslant \phi \leqslant 330 \mathrm{rad} \mathrm{m}^{-2}$ in steps of $\delta \phi=0.001 \mathrm{rad} \mathrm{m}^{-2}$ to oversample the FDF in Faraday space. The largest RM to which we have more than $50 \%$ sensitivity to, at the centre observing frequency, is $\left|\phi_{\max }\right|=\sqrt{3} / \delta\left(\lambda^{2}\right)=163 \mathrm{rad} \mathrm{m}^{-2}$. This quantity is dictated primarily by the observing channel width in wavelength squared $\delta\left(\lambda^{2}\right)$ (Equation 63 from Brentjens \& de Bruyn 2005). The RM range of $\pm 330 \mathrm{rad} \mathrm{m}^{-2}$ was computed for the FDFs because this is twice the $\left|\phi_{\max }\right|$ value. This also provided a large range in $\phi$ with which to calculate the rms noise value. An alternative method for calculating this quantity from Schnitzeler \& Lee (2015) gives a somewhat similar expected value of $\left|\phi_{\max }\right|=125 \mathrm{rad} \mathrm{m}^{-2}$. See Table 1 for a summary of the observation and RM-synthesis parameters.

After we obtained an FDF for each pulsar, the location of the peak in Faraday space was determined and fitted using a quadratic function, providing a measurement of the RM. This was regarded as significant if the peak signal-to-noise in the FDF was greater than 4, $\mathrm{S} / \mathrm{N}_{F}>4$. This threshold was chosen because after conducting RMsynthesis with several examples of noise as the input (i.e. off-pulse data), we found that all of the peaks in the FDFs had a $\mathrm{S} / \mathrm{N}_{F} \leqslant 3.8$, although the large majority had $\mathrm{S} / \mathrm{N}_{F}<3$.

Any possible instrumental polarisation response around $0 \mathrm{rad}$ $\mathrm{m}^{-2}$ (due to no wavelength-squared dependency) was excised from the FDFs. Instrumental polarisation can result from, for example, low levels of RFI that have not been excised in the previous data reduction steps, or from 'leakage', where emission from Stokes I 'leaks' into the other Stokes parameters, causing the mean values of Stokes $Q, U$ to be unequal. Stokes $Q$ is generally most affected by leakage because it is formed from the same polarisation bases as Stokes $I$. This leakage can somewhat be reduced by polarisation calibrating the data, using a model for the primary beam, for example. However, the beam models and other empirical corrections used for low-frequency aperture-array telescopes such as LOFAR cannot currently completely remove this polarisation leakage (e.g. Lenc et al. 2017). A major advantage in using low-frequency data to obtain RMs is that the FWHM of the RMSF is narrow, enabling identification and separation of the peaks resulting from any instrumental polarisation and the desired astronomical RM signal in the

\footnotetext{
7 https://github.com/gheald/RMtoolkit
} 
Table 1. Summary of LOFAR HBA data used in this work and corresponding theoretical RM-synthesis parameters.

\begin{tabular}{llr}
\hline \hline Parameter & Symbol & Data \\
\hline Centre frequency & $v$ & $148.9 \mathrm{MHz}$ \\
Bandwidth & $\Delta v$ & $78.1 \mathrm{MHz}$ \\
Channel width (averaged) & $\delta v$ & $195 \mathrm{kHz}$ \\
Centre wavelength squared & $\lambda^{2}$ & $4.0 \mathrm{~m}^{2}$ \\
Total bandwidth in wavelength squared $\left(\lambda_{\max }^{2}-\lambda_{\min }^{2}\right)$ & $\Delta\left(\lambda^{2}\right)$ & $4.9(7.4-2.5) \mathrm{m}^{2}$ \\
Resolution in Faraday space (FWHM of the RMSF) & $\delta \phi$ & $0.7 \mathrm{rad} \mathrm{m}^{-2}$ \\
Largest scale in Faraday space to which one is sensitive & $\max$-scale/ $\Delta \phi$ & $1.2 \mathrm{rad} \mathrm{m}^{-2}$ \\
Maximum observable Faraday depth (at $\nu_{\min }, \nu, v_{\max }$, respectively) & $\left|\phi_{\max }\right|$ & $66,163,327 \mathrm{rad} \mathrm{m}^{-2}$ \\
\hline
\end{tabular}
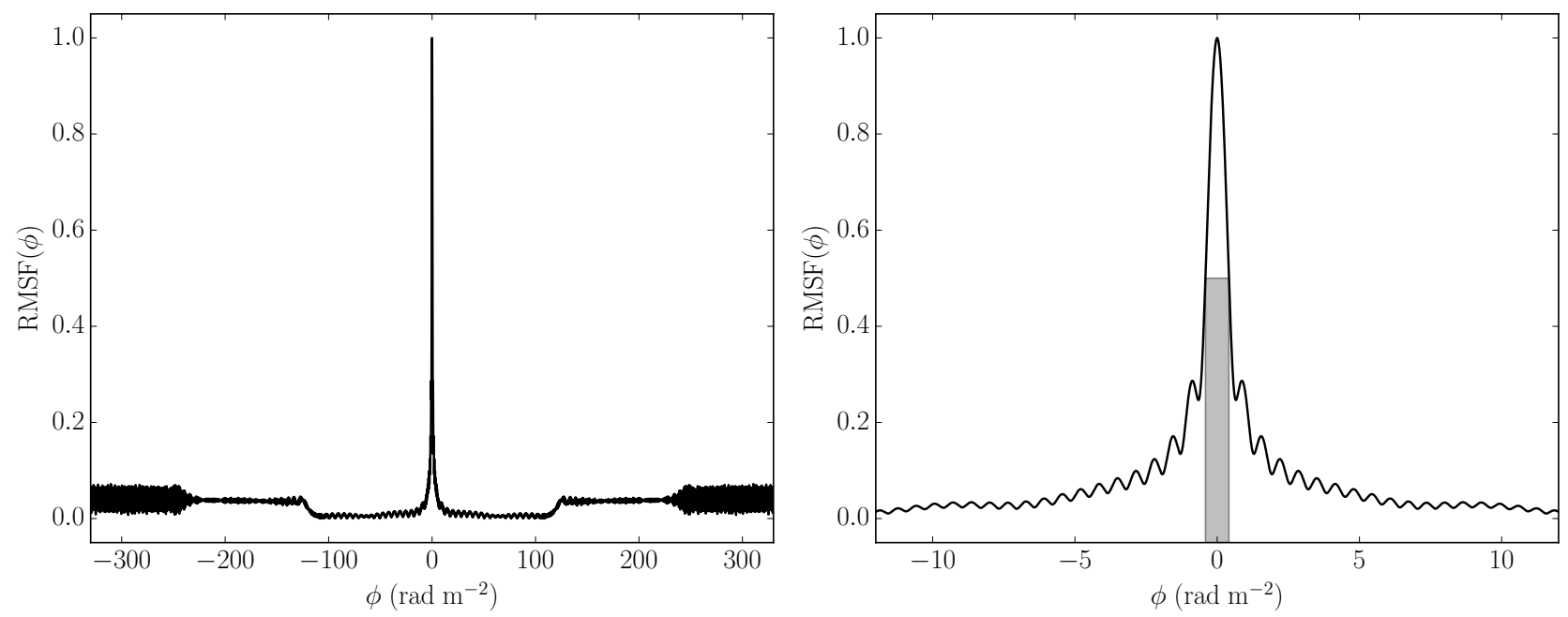

Figure 1. Theoretical, noiseless RMSF expected from RM-synthesis using the LOFAR data parameters summarised in Table 1, shown for both large (left) and narrow (right) ranges in Faraday space. Right: The resolution in Faraday space, $\delta \phi$, is also shown by the FWHM of the RMSF shaded in grey.

FDF. For the LOFAR data presented in this work, the theoretical FWHM of the RMSF is $\delta \phi=3.8 / \Delta\left(\lambda^{2}\right) \approx 0.7 \mathrm{rad} \mathrm{m}^{-2}$ (Equation 61 in Brentjens \& de Bruyn 2005; Schnitzeler et al. 2009). This small value for $\delta \phi$ facilitates the precise measurement of RMs, see Figures 1 and 2. For the FDFs obtained in this work, we excised the signal within $\pm \delta \phi$ around $0 \mathrm{rad} \mathrm{m}^{-2}$, i.e., $-0.7<\phi<0.7 \mathrm{rad}$ $\mathrm{m}^{-2}$. This allows the measurement of absolute RMs above $\approx 0.7 \mathrm{rad}$ $\mathrm{m}^{-2}$, which applies to 99 per cent of the current pulsar catalogue. In addition, the absolute ionospheric RM is often on the order of $\approx 1 \mathrm{rad} \mathrm{m}^{-2}$, see Section 2.2. We measured the location of the peak in the FDFs due to the instrumental polarisation near $0 \mathrm{rad} \mathrm{m}^{-2}$, and a histogram of the results are shown in Figure B2. We find that the instrumental peaks in the FDFs do not deviate greatly from $0 \mathrm{rad}$ $\mathrm{m}^{-2}$.

The formal RM uncertainty was determined by measuring the FWHM of the signal peak in the FDF (i.e. the measured value for $\delta \phi ; \mathrm{FWHM}_{F}$ ), as well as the $\mathrm{S} / \mathrm{N}_{F}$. The rms noise in the FDF was calculated across the search range in RM space, excluding the peak(s) associated with the pulsar signal and the instrumental response near $0 \mathrm{rad} \mathrm{m}^{-2}$. The formal $\mathrm{RM}$ uncertainty was calculated as

$\frac{\text { FWHM }_{\mathrm{F}}}{2 \times \mathrm{S} / \mathrm{N}_{\mathrm{F}}}$,

following Brentjens \& de Bruyn (2005); Schnitzeler \& Lee (2017). If no significant peak in the FDF was detected, i.e., $\mathrm{S} / \mathrm{N}_{F}<4$, attempts to increase the $\mathrm{S} / \mathrm{N}_{F}$ were made by extracting the integrated Stokes parameters for an increasingly large range of the on-pulse phase bins in the average pulse profile, up to the full width at 10 per cent of the maximum of the profile. The total number of phase bins in the pulsar profiles is mostly 1024, but range from 64 to 1024 in powers of 2, and are listed in Table A1. The median number of phase bins used to extract the Stokes parameters was 3 for the 'slow' pulsar census and 8 for the MSP census, these are also listed for each pulsar in Table A1. The total number of bins summed were increased gradually to minimise possible effects of varying polarisation angle and/or RM across the pulse profile (e.g. Noutsos et al. 2015), which will be investigated in future work. For FDFs with $4<\mathrm{S} / \mathrm{N}_{F}<8$, the formal error was multiplied by 2 , to reflect the larger uncertainties on the lower S/N detections. This was approximately the deviation in the RMs obtained using a subset of the pulsar data, with multiple ranges in the pulse profile bins to extract the Stokes parameters, see Appendix B for further discussion and Figure B1 for high and low S/N examples. FDFs with $\mathrm{S} / \mathrm{N}_{F} \geqslant 8$ have been shown to be reliable (e.g. George et al. 2012). Although RMs can also be measured as a function of pulse phase space, low-frequency observations indicate much less deviation across the pulse profile compared to higher-frequency data (e.g. Noutsos et al. 2009, 2015). Pulsar observations where between one and five phase bins were used to measure the RMs are good candidates for phase-resolved RM studies in future work.

The RM-synthesis was coupled with RM CLEAN ${ }^{7}$ analysis to deconvolve the FDF using the theoretical RMSF (Heald et al. 2009; Michilli et al. 2018c). RM CLEAN allowed us to estimate the intrinsic Faraday spectrum, to obtain the second moment of the RM CLEAN components (RM dispersion; $\sigma_{\mathrm{RM}}$ ), and to investigate the extent to which the FDFs obtained are Faraday thin (e.g. Heald 


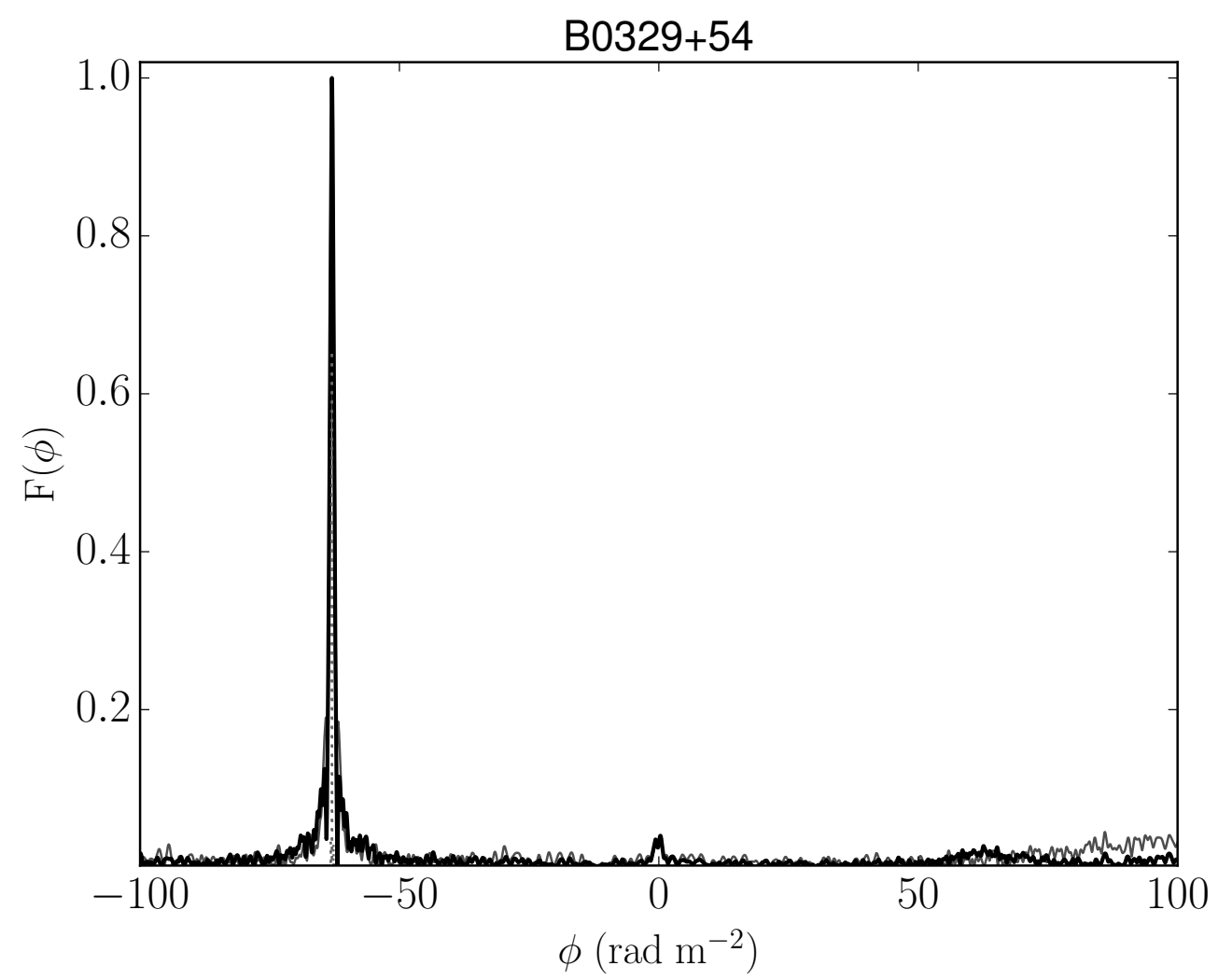

Figure 2. An example Faraday dispersion function (FDF; grey line) using a 10-minute LOFAR HBA observation towards PSR B0329+54 centred at 149 MHz. The vertical axis is in arbitrary units (normalised) because the data are not flux or polarisation calibrated, see text for further details. The black line shows the resulting spectrum produced after ten iterations of RM CLEAN, stopping at the specified $8 \times$ rms threshold. The dotted grey lines show the locations of the RM CLEAN components centred at $\mathrm{RM}_{\mathrm{obs}}=-63.016 \pm 0.005 \mathrm{rad} \mathrm{m}^{-2}$, before correcting for the ionospheric RM. A small amount of instrumental polarisation ( 3 per cent) is evident near $0 \mathrm{rad} \mathrm{m}^{-2}$. A small amount of leakage to $+63 \mathrm{rad} \mathrm{m}^{-2}$ and some RMSF structure at larger RM values can also be seen in the baseline.

et al. 2009; Anderson et al. 2015). Faraday thickness can originate in volumes of plasmas with regular magnetic fields that both emit and Faraday rotate, or in plasmas with turbulent magnetic fields that Faraday rotate, leading to polarised flux being distributed over a range of Faraday depths (Burn 1966). A Faraday thin source satisfies $\lambda^{2} \Delta \phi \ll 1$, where $\Delta \phi$ is the extent of the source in Faraday space (Brentjens \& de Bruyn 2005). FDFs calculated from lowfrequency data provide the tightest constraint on $\Delta \phi$ because of the large $\lambda^{2}$, which is $4.9 \mathrm{~m}^{2}$ for the LOFAR data in this work, see Table 1. The largest scale in Faraday space to which one is sensitive, using these LOFAR data, is $\pi / \lambda_{\text {min }}^{2}=1.2 \mathrm{rad} \mathrm{m}^{-2}$ (Equation $62 \mathrm{in}$ Brentjens \& de Bruyn 2005). Pulsars are not expected to be Faradayresolved (-thick) sources because they are point sources and their magnetospheric emission is not expected to impart Faraday rotation, in particular with the usual wavelength squared dependency, due to the relativistic electron-positron pair plasma expected in their magnetospheres (e.g. Wang et al. 2011). The degree of polarisation emitted often increases towards lower frequencies (e.g. Johnston et al. 2008; Noutsos et al. 2015), which is also contrary to that expected from a Faraday thick source that becomes substantially depolarised towards longer wavelengths. Despite this expectation, the RM dispersion of pulsars has not been widely explored in the literature.

In this work, the polarisation data were normalised by a powerlaw model characterised by a spectral index, $\alpha$, that was fitted to the apparent Stokes I spectrum $\left(\mathrm{S}(v) \propto v^{-\alpha}\right)$. For each pulsar, the FDF was computed and, if a significant RM was detected, we RM
CLEANed down to $3 \times$ rms of the noise in the FDF. Any peak in the FDF near $0 \mathrm{rad} \mathrm{m}^{-2}$ caused by instrumental polarisation was ignored in this analysis.

Using the method described above, a single number for the RM (and RM dispersion) towards each pulsar was obtained for the purpose of studying the GMF, and are summarised in Table A1. All uncertainties are labelled ' \pm ' in this paper, to avoid confusion with the RM dispersion, $\sigma_{\mathrm{RM}}$. Pulsars with published RMs in the pulsar catalogue were cross-checked with the results from this work to verify and compare the (low-frequency) RMs obtained, see Section 3. Future work will present the calibrated polarisation profiles and phase-resolved RMs, in order to further investigate, e.g., the pulsar emission mechanism.

\subsection{Ionospheric Faraday rotation subtraction}

The ionosphere, also a magneto-ionic plasma, introduces an additional RM that is both time and position dependent. Therefore, the ionospheric $\mathrm{RM}, \mathrm{RM}_{\mathrm{ion}}$, must be subtracted from the observed $\mathrm{RM}$, $\mathrm{RM}_{\mathrm{obs}}$, in order to obtain a measurement of the RM due to the ISM alone, i.e., $\mathrm{RM}_{\mathrm{ISM}}=\mathrm{RM}_{\mathrm{obs}}-\mathrm{RM}_{\mathrm{ion}}$.

The $\mathrm{RM}_{\text {ion }}$ towards the $\mathrm{LoS}$ (at the corresponding ionospheric pierce point, IPP) at the time of each observation was calculated by using a previously tested and verified code, IONFR ${ }^{8}$ (see Sotomayor-

8 https://sourceforge.net/projects/ionfarrot/ 
Beltran et al. 2013). This code models the $\mathrm{RM}_{\mathrm{ion}}$ using vertical total electron content (VTEC) maps of the ionosphere (obtained using the distribution of worldwide GPS stations) plus a standard mathematical description of the Earth's main magnetic field. Several pulsar observations taken over the course of several hours have previously been used to investigate the $\mathrm{RM}_{\mathrm{ion}}$ - estimated using several publicly available VTEC maps ${ }^{9}$ as inputs (e.g. SotomayorBeltran et al. 2013; Sobey 2015). The CODE (e.g. Dach et al. 2018) and IGS (e.g. Hernández-Pajares et al. 2009) VTEC maps used to produce $\mathrm{RM}_{\mathrm{ion}}$ estimates were found to provide a good fit to the $\mathrm{RM}_{\mathrm{obs}}$. In this work, the IGS TEC maps were used because they generally have smaller uncertainties (calculated using the VTEC rms maps), which are the largest contribution to the uncertainty in $\mathrm{RM}_{\mathrm{ion}}$. For this work, we also updated the IonFR code to use the most recent version of the International Geomagnetic Reference Field $^{10}$ (IGRF-12; Thébault et al. 2015). Comparing the IONFR output between using the previous and current versions of the IGRF (11 or 12 , respectively), the $\mathrm{RM}_{\text {ion }}$ values differ by less than 2 per cent of the uncertainties (in the cases of several pulsar LoSs tested). IGRF-12 was used because it is the most recent release and provides the geomagnetic field components beyond the year 2015 .

In this work, we also investigated the repeatability of this method for estimating $\mathrm{RM}_{\text {ion }}$ over a longer timespan ( $\approx$ year), and the accuracy of the resulting RM $_{\text {ISM }}$ measurements that can be expected. Ten (timing/monitoring) observations of a bright, northern pulsar $\mathrm{B} 0329+54$, were used to compare the $\mathrm{RM}_{\mathrm{obs}}$ and $\mathrm{RM}_{\text {ion }}$ over the course of nine months from 2014 February 3 to 2014 November 3. These observations used the same parameters as those shown in Table 1. We measured the DM for each observation using the PDMP routine in PSRCHIVE and corrected the archive file for this value. We then measured the $\mathrm{RM}_{\mathrm{obs}}$ using the method described in Section 2.1. We used the IGS VTEC maps and IGRF-12 as inputs to the IONFR code to estimate $\mathrm{RM}_{\text {ion }}$ for each average observation time (and corresponding IPP) for PSR B0329+54. We do not apply the ionospheric RM correction to the pulsar archive files on timescales shorter than one observation. This is because the data are not severely depolarised over the short integration time (just $\approx 10-20$ minutes in length) and the pulsar signals are often highly polarised. Observations could be corrected on shorter timescales, if particularly active ionospheric conditions dictate that this is necessary. However, the IGS VTEC maps we use have a time resolution of 2 hours (interpolated to every hour in the IONFR code) and we find that a single $\mathrm{RM}_{\mathrm{ion}}$ correction per observation is sufficient for this work. We corrected $\mathrm{RM}_{\mathrm{obs}}$ using the $\mathrm{RM}_{\text {ion }}$ values output from IONFR, and also calculated the inferred electron-density-weighted $\left\langle B_{\|}\right\rangle$using Equation 1. The results are shown in Figure 3.

The weighted mean of the DM measurements of PSR B0329+54, shown in Figure 3, is $26.7624 \mathrm{pc} \mathrm{cm}^{-3}$ with a standard deviation of $0.0008 \mathrm{pc} \mathrm{cm}^{-3}$. The DM measurements are not corrected for the ionospheric DM because the ionosphere imparts a negligible amount of DM compared to the measurement uncertainties here. Figure 3 shows that the variation in the $\mathrm{RM}_{\text {ion }}$ estimates appears to be a good fit to that for the $\mathrm{RM}_{\mathrm{obs}}$ measurements. The weighted mean of the $\mathrm{RM}_{\text {ISM }}$ values is $-64.33 \mathrm{rad} \mathrm{m}^{-2}$ with standard deviation $0.06 \mathrm{rad} \mathrm{m}^{-2}$. This $\mathrm{RM}_{\mathrm{ISM}}$ value is in agreement with $(<1.4 \times$ uncertainties), and approximately 7 -times more precise than, the literature value from the pulsar catalogue: $\mathrm{RM}_{\mathrm{cat}}=-$

9 available at NASA's Archive of Space Geodesy Data, retrieved from $\mathrm{ftp} / /$ cddis.nasa.gov/pub/gps/products/ionex/.

${ }_{10} \mathrm{https} / / /$ www.ngdc.noaa.gov/IAGA/vmod/igrf.html

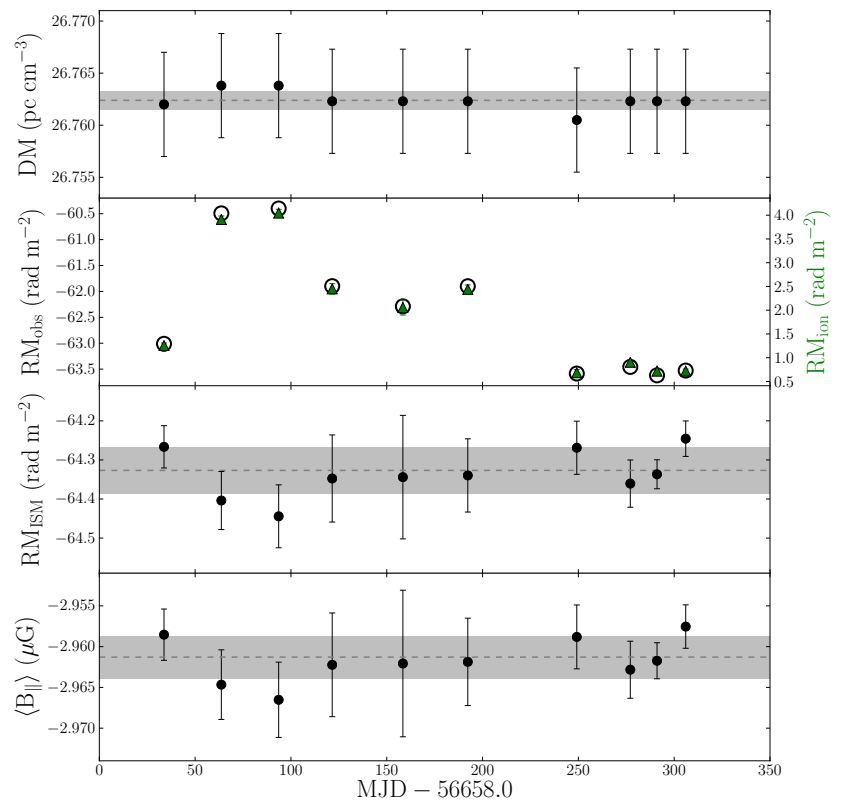

Figure 3. Measurements using the LOFAR timing observations of PSR B0329+54 centred at $149 \mathrm{MHz}$ with $78 \mathrm{MHz}$ bandwidth. From the upper to lower panels we show the following quantities plotted against Modified Julian Date from 2014 January $1(\mathrm{MJD}=56658)$ : the measured DMs; the observed $\mathrm{RMs}\left(\mathrm{RM}_{\mathrm{obs}}\right.$; open circles; left y-axis) and the estimated ionospheric $\mathrm{RM}\left(\mathrm{RM}_{\mathrm{ion}}\right.$; green triangles; right y-axis) and their uncertainties; the $\mathrm{RM}_{\mathrm{ISM}}$ calculated from each $\mathrm{RM}_{\mathrm{obs}}-\mathrm{RM}_{\mathrm{ion}}$; and the electron density-weighted average magnetic field parallel to the LoS, calculated using Equation 1 and the DM and RMISM. The weighted means of the relevant measurements are shown by the grey dashed lines, and one standard deviation from the mean is shown by the grey shaded areas.

$63.7 \pm 0.4 \mathrm{rad} \mathrm{m}^{-2}$. The catalogue value was measured using observations at several centre frequencies between $250-500 \mathrm{MHz}$, and taken over 40 years before the LOFAR observations (Manchester 1972). Manchester (1972) corrected the data for $\mathrm{RM}_{\text {ion }}$ using continuous records of the total ionospheric Faraday rotation obtained towards a geostationary satellite. We also calculated the weighted mean of the $\left\langle B_{\|}\right\rangle$values, $-2.961 \mu \mathrm{G}$, with standard deviation $0.003 \mu \mathrm{G}$. This is also in good agreement (within 1.3 times the uncertainties) with the value published in Manchester (1972). In addition, we found that the median RM dispersion for all of the FDFs to be $\sigma_{\mathrm{RM}}=0.0005 \mathrm{rad} \mathrm{m}^{-2}$, which is Faraday thin. The RM dispersions for the whole pulsar set are further discussed in Section 3.1 .

The mean uncertainties on the values for $\mathrm{RM}_{\mathrm{obs}}$ and $\mathrm{RM}_{\mathrm{ion}}$ are 0.01 and $0.08 \mathrm{rad} \mathrm{m}^{-2}$, respectively. This is an example of the excellent precision of the measurements that can be obtained from using the low-frequency LOFAR data. This also indicates that the $\mathrm{RM}_{\text {ISM }}$ uncertainty is dominated by the current method for correcting $\mathrm{RM}_{\text {ion }}$. The data in Figure 3, along with previous work, show that we can expect reasonably high accuracy in the measurement of $\mathrm{RM}_{\mathrm{ISM}}$ using the current method for correcting for $\mathrm{RM}_{\mathrm{ion}}$, e.g., the

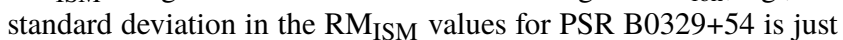
$0.06 \mathrm{rad} \mathrm{m}^{-2}$. However, it is clear that more sophisticated methods that are being developed for more accurately determining $\mathrm{RM}_{\text {ion }}$ will be essential towards fully realising the RM precision possible using low-frequency data (e.g. Malins et al. 2018), in particular for the LOFAR Low-Band Antenna observations. This will also allow us to fully realise higher $\left\langle B_{\|}\right\rangle$precision, since the fractional un- 
certainties on the RM measurements are currently a factor of $\approx 100$ larger than the fractional uncertainties on the DM measurements.

The results published in Table A1 include the $\mathrm{RM}_{\mathrm{obs}}$ measured, the $\mathrm{RM}_{\text {ion }}$ estimates, and the resulting $\mathrm{RM}_{\mathrm{ISM}}$ and uncertainties, along with the dates and times of the observations. This is to provide the possibility of applying more advanced ionospheric Faraday rotation corrections that may become available in the future.

\section{RESULTS}

Here, we summarise the results of the RMs determined towards pulsars using the LOFAR observations described.

We determined the RMs towards 117 pulsars in the nonrecycled pulsar census and 19 pulsars in the MSP census (136 in total), presented in Table A1. This represents 74 and 40 per cent of all of the pulsars detected in each of the LOFAR censuses, respectively, and 86 and 60 per cent of the pulsars with total intensity S/N greater than 10 , respectively. The pulsars in these censuses were not selected based on their polarisation characteristics (but on position accuracy, Galactic location, see Bilous et al. 2016; Kondratiev et al. 2016). Therefore, they form a reasonably representative sample of the proportion of pulsars that can be used to measure RMs below $200 \mathrm{MHz}$. The FDFs obtained towards each pulsar are shown in Appendix B.

The pulsars with RMs measured in this work are located between 14 and 321 degrees in Galactic longitude (although the majority are between 20-200 degrees) and -58 and 86 in Galactic latitude. The pulsar closest to the Galactic plane is PSR B1937+21, at Galactic latitude -0.29 degrees. The DM range is between 3 and $161 \mathrm{pc} \mathrm{cm}^{-3}$ (PSRs J1744-1134 and B2036+53, respectively). The $\mathrm{RM}_{\text {ISM }}$ range is between $-168.7 \pm 0.1$ and $154.9 \pm 0.2 \mathrm{rad} \mathrm{m}^{-2}$ (PSRs B2210+29 and B1848+13, respectively). The smallest absolute RM measurement, $\mathrm{RM}_{\mathrm{ISM}}=0.96 \pm 0.09 \mathrm{rad} \mathrm{m}^{-2}$, is towards PSR $\mathrm{J} 0435+2749$. The range in average magnetic field strength parallel to the LoS calculated is between $-5.635 \pm 0.009$ and $3.524 \pm 0.004 \mu \mathrm{G}$ (for PSRs J2043+2740 and B1842+14, respectively). The uncertainties on these measurements provide examples of the accuracy that can be obtained for $\left\langle B_{\|}\right\rangle$, towards studying the Galactic magnetic field using the methods described.

Distance estimates for the pulsars (those without independent distance measurements) are calculated using the recently published Galactic electron density model from Yao et al. (2017), hereafter referred to as YMW16 ${ }^{11}$, this is further discussed in Section 4.5. The distance estimates for pulsars with RM measurements range from 0.2 to $25 \mathrm{kpc}$ (although the few pulsars with $25 \mathrm{kpc}$ distances are likely overestimated, as this is the limit given by the model, see Section 4.5). The distances of the pulsars from the Galactic plane used in the analysis of the magnetic scale height of the Galactic halo is between -3 and $4 \mathrm{kpc}$, see Section 4.4 .

\subsection{RM dispersion results}

Figure 4 summarises the RM dispersion, $\sigma_{\mathrm{RM}}$, calculated by running RM CLEAN on the FDFs obtained towards each pulsar. The RM dispersion results for each pulsar are also included in Table A1. The RM dispersion bin with the largest number of pulsars (63) represents values less than $0.0009 \mathrm{rad} \mathrm{m}^{-2}$. This is $<1$ per cent of the typical $\mathrm{RM}$ uncertainty. Eight pulsars with $\mathrm{S} / \mathrm{N}_{F}>7$ have $\mathrm{RM}$ dispersions

11 www.xao.ac.cn/ymw16/

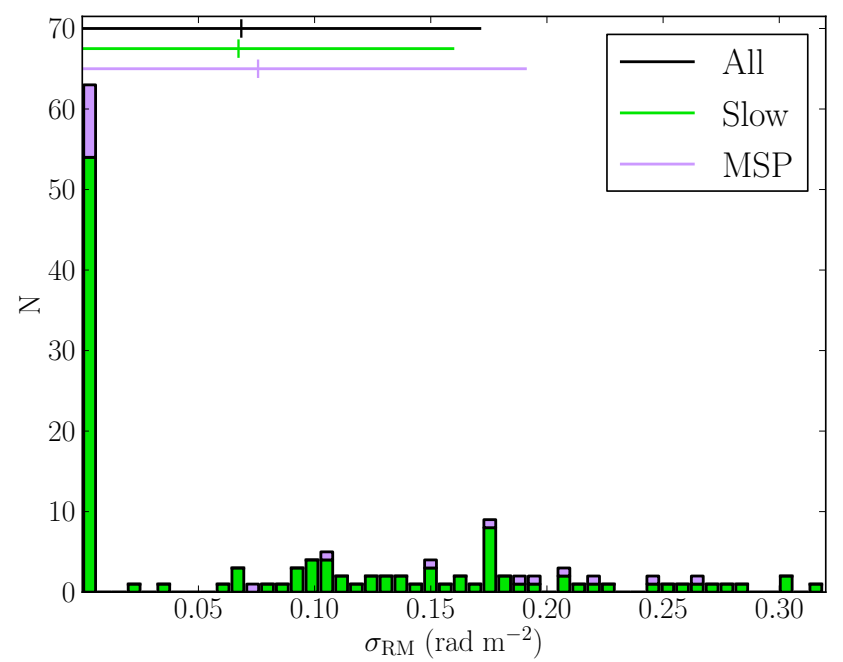

Figure 4. A stacked histogram showing the RM dispersion $\left(\sigma_{R M}\right)$ for the pulsars in the 'slow' pulsar (green) and MSP (purple) censuses. The horizonal lines with corresponding colours above mark the range between the 25 th and 75 th percentiles, with the median shown by the vertical line (the complete pulsar set is shown by the black line).

that are indistinguishable from $0 \mathrm{rad} \mathrm{m}^{-2}$, i.e., all of the components from RM CLEAN fall within the same pixel value after at least one RM CLEAN iteration. These are PSRs B0331+45, B0523+11, $\mathrm{J} 0611+30$, B0940+16, J1612+2008, J1645+1012, J1741+2758, and B2110+27. The median RM dispersion for the RMs in this work (from both censuses) is $0.068 \mathrm{rad} \mathrm{m}^{-2}$, which is less than 10 per cent of $\delta \phi$, see Table 1. Furthermore, all of the values obtained are less than 40 per cent of $\delta \phi$. The RM dispersions of 75 per cent of the pulsars in this work are less than $0.17 \mathrm{rad} \mathrm{m}^{-2}$ and satisfy the Faraday thin criterion, $\lambda^{2} \Delta \phi \ll 1$, even at the longest observing wavelength $\left(\lambda^{2}=7.4 \mathrm{~m}^{2}\right)$. For the few pulsars that have larger RM dispersions, it is likely that this is largely due to RM CLEAN-ing too deeply in the presence of noise. All of the pulsars in this work satisfy $\lambda^{2} \Delta \phi \ll 1$ at the shortest observing wavelength $\left(\lambda^{2}=2.5 \mathrm{~m}^{2}\right)$. In addition, there is no evidence for emission at more than one RM value to a high degree of confidence, also as expected in FDFs obtained using pulsar data.

We investigated possible correlations between the RM dispersion and a number of parameters. We found no significant correlation between the RM dispersion and: the number of bins used to extract the Stokes parameters from the pulse profiles; the S/N in the FDFs; the DMs; the absolute value of the measured RMs; or the uncertainty on the RMs. We also obtained published scattering measures, $\tau_{\mathrm{sc}}$, at $1 \mathrm{GHz}$ from the pulsar catalogue for 54 of the slow pulsars and 3 of the MSPs (Manchester et al. 2005, and references therein) to investigate any correlation with the RM dispersion. Again, we found no significant correlation trend between these two variables. However, two pulsars with the largest scattering times also have among the largest RM dispersions measured. These are the Crab pulsar PSR B0531+21 with $\tau_{\mathrm{sc}}=1.2 \times 10^{-4} \mathrm{~s}$ at $1 \mathrm{GHz}$ and $\sigma_{\mathrm{RM}}=0.25 \mathrm{rad} \mathrm{m}^{-2}$; and PSR B1946+35 with $\tau_{\mathrm{sc}}=4.1 \times 10^{-4} \mathrm{~s}$ at $1 \mathrm{GHz}$ and $\sigma_{\mathrm{RM}}=0.30 \mathrm{rad} \mathrm{m}^{-2}$, see Section 4.3 for further discussion for the Crab pulsar. Whether some of the larger RM dispersions may in fact be the result of foreground diffuse ISM structures affecting the signal will require a more comprehensive study, using the calibrated polarisation profiles as a function of observing frequency.

The RM dispersions obtained using these LOFAR data show 
that the majority of the signals from the pulsars are extremely Faraday thin, as we expected. These low-frequency observations provide the most stringent information about the extent to which pulsars are Faraday thin, to date.

\section{DISCUSSION}

Here, we discuss the RM results that we obtained using the LOFAR observations and provide further analysis in combination with literature RM measurements. In Section 4.1 we examine the nature of the data for pulsars towards which we did not detect a significant RM. In Section 4.2 we compare the RMs that we measured at lowfrequencies to measurements from the literature towards the same pulsars, as well as towards extragalactic sources along the same LoSs to provide further context. In Section 4.3 we briefly comment on the results for some individual pulsars. In Section 4.4 we further analyse the RMs towards pulsars to give estimates of the magnetic field scale height in the Galactic halo. In Section 4.5 we discuss the limitations of the data we currently have to study the Galactic magnetic field structure, and prospects for future improvements.

\subsection{Detecting and measuring RMs}

For the pulsars where we did not detect a significant RM with $\mathrm{S} / \mathrm{N}_{F}>4$, the primary reason seems to be lower $\mathrm{S} / \mathrm{N}$ in the pulse profile (resulting in lower $\mathrm{S} / \mathrm{N}_{\mathrm{P}}$ in the pulse profile). The median $\mathrm{S} / \mathrm{N}$ for the slow pulsars and MSPs that we did detect an RM for are 67 and 19, respectively. Meanwhile, the slow pulsars and MSPs that we did not detect an RM for have median S/N of 10 and 8, respectively. However, the lowest total intensity pulse profile $\mathrm{S} / \mathrm{N}$ for which we did detect a significant RM is just 4 (for PSR J2002+1637), as this pulsar is highly polarised. For 14 pulsars (10 slow and 4 MSPs), we detected a tentative RM in the FDFs, but these were below the chosen $\mathrm{S} / \mathrm{N}$ threshold set, $\mathrm{S} / \mathrm{N}_{F}>4$, and so are not included in the final catalogue. Half of these pulsars do not have an RM published in the literature, and it is likely that a significant RM would be detected if a longer integration time is used to increase the $\mathrm{S} / \mathrm{N}$ in the average pulse profiles. Furthermore, the median DM of the pulsars with significant RMs is $35 \mathrm{pc} \mathrm{cm}^{-3}$, while the median DM of the pulsars with no significant RM detection is almost double: $61 \mathrm{pc} \mathrm{cm}^{-3}$. For the 'slow' pulsars, this is due to larger distances to the pulsars, which also reduces the flux density: the median distance estimates for pulsars with or without a significant RM detection are $2.2 \mathrm{kpc}$ and $3.9 \mathrm{kpc}$, respectively. For the MSPs, this is not necessarily the case: the median distance estimates for MSPs with or without a significant RM detection are $1.2 \mathrm{kpc}$ and $1.3 \mathrm{kpc}$, respectively. Therefore, is likely that dispersion smearing and/or scattering in some of the pulse profiles may also have an effect on the $\mathrm{S} / \mathrm{N}$ and degree of polarisation of the signals, see below. The $\mathrm{S} / \mathrm{N}_{\mathrm{P}}$ could be increased for pulsars that have been observed at least once (e.g. for timing/monitoring campaigns) by adding the datasets together (after correcting for ionospheric RM variations).

Several of the pulsars show evidence of scattering tails, in some extreme cases these increase to span almost the entire pulse phase (see Bilous et al. 2014; Geyer et al. 2017). Whether we can detect an RM in these data seems to vary on a case by case basis. In some cases where the pulsar is reasonably polarised at low frequencies we detected a significant RM, although more pulse phase bins may need to be summed (e.g. for PSR B1946+35 we summed 70 phase bins, or 7 per cent of the rotational period, to gain sufficient $\mathrm{S} / \mathrm{N}$ ). For
Table 2. Summary of pulsars towards which we did not detect a significant RM in this work, and which also do not have an RM published in the literature.

\begin{tabular}{llll}
\hline \hline PSR name & PSR name & PSR name & PSR name \\
\hline 'Slow' census & $=15$ & & \\
\hline J0006+1834 & J1834+10 & J1908+2351 & J2040+1657 \\
J0324+5239 & J1848+0826 & J1913+3732 & J2048+2255 \\
J0329+1654 & J1900+30 & J1937+2950 & J2243+1518 \\
J0711+0931 & J1901+1306 & B2025+21 & \\
\hline MSP census & $=14$ & & \\
\hline J0337+1715 & J1544+4937 & J1905+0400 & J2215+5135 \\
J1023+0038 & J1709+2313 & J1918-0642 & J2302+4442 \\
J1038+0032 & J1738+0333 & B1957+20 & \\
J1231-1411 & J1816+4510 & J2019+2425 & \\
\hline
\end{tabular}

others, the scattering may contribute to depolarising the signal towards low frequencies, e.g., PSR B2053 +36 has RM $_{\text {cat }}=-68.00 \mathrm{rad}$ $\mathrm{m}^{-2}$, but we were unable to detect a significant RM at our observing frequency. Furthermore, 29 of the pulsars towards which we did not detect a significant RM also do not have an RM published in the literature, listed in Table 2. It may be that the signal from some of these pulsars is intrinsically unpolarised, possibly due to the emissionbeam-LoS geometry, orthogonal polarisation modes, or spin-down luminosity (e.g. Radhakrishnan \& Cooke 1969; Manchester et al. 1975; Johnston \& Kerr 2018).

For six pulsars, the peak in the FDF was within the range set for any expected instrumental polarisation $\left(-0.77<\phi<0.77 \mathrm{rad} \mathrm{m}^{-2}\right)$ and since we cannot (using the current data processing methods) be confident whether the majority of the polarised signal is from the pulsar or the instrument, these results were not included in our catalogue. Most of these pulsars have small RM values (e.g. for PSR $\mathrm{B} 1237+25 \mathrm{RM}_{\mathrm{cat}}=-0.12 \pm 0.06 \mathrm{rad} \mathrm{m}^{-2}$ ) or have large fractional uncertainties (e.g. for PSR J1503+2111 RM $\mathrm{RM}_{\mathrm{cat}}=-5 \pm 10 \mathrm{rad} \mathrm{m}^{-2}$ ). Limits on the RMs for these pulsars could have been included by using the ionospheric RM calculated (mostly less than $1 \mathrm{rad}$ $\mathrm{m}^{-2}$ ). However, we can obtain more reliable measurements by reobserving these pulsars when the ionosphere is more ionised and imparts a more substantial $\mathrm{RM}_{\mathrm{obs}}$, e.g. during the day.

Six of the pulsars for which we did not detect a significant RM have absolute catalogued RMs larger than the expected maximum observable RM (i.e. $\pm 163 \mathrm{rad} \mathrm{m}^{-2}$, where $\approx 50$ per cent sensitivity is lost due to bandwidth depolarisation, at the central observing frequency, see Table 1). The largest absolute RMs that we measured are approximately equal to this value: PSR J2017+2043 with $\mathrm{RM}_{\text {obs }}=-$ $160.93 \mathrm{rad} \mathrm{m}^{-2}$ and PSR B2210+29 with $\mathrm{RM}_{\mathrm{obs}}=-165.23 \mathrm{rad} \mathrm{m}^{-2}$. This is because the upper half of the bandwidth (149-188 MHz) can still be used to detect a significant signal (albeit with a slightly larger $\mathrm{FWHM}_{F}$ ). For these pulsars, the RM measurement was verified and the $\mathrm{S} / \mathrm{N}_{F}$ increased by downloading higher frequency resolution data, available from the LOFAR Long-Term Archive (down to $30 \mathrm{kHz}$ channel width). These data raise the maximum expected observable RM to $\pm 1000 \mathrm{rad} \mathrm{m}^{-2}$ at the central observing frequency. The higher frequency resolution data was not used for all of the census pulsars because this is not necessary for pulsars with lower absolute RMs (this applies to the majority of the pulsars in the sample) and the data volume is over 6 times larger, increasing the download and processing times. We also downloaded the higher frequency resolution data and obtained FDFs for the six pulsars with larger catalogued RMs, but none resulted in a significant detection using the standard or higher frequency resolution data. 


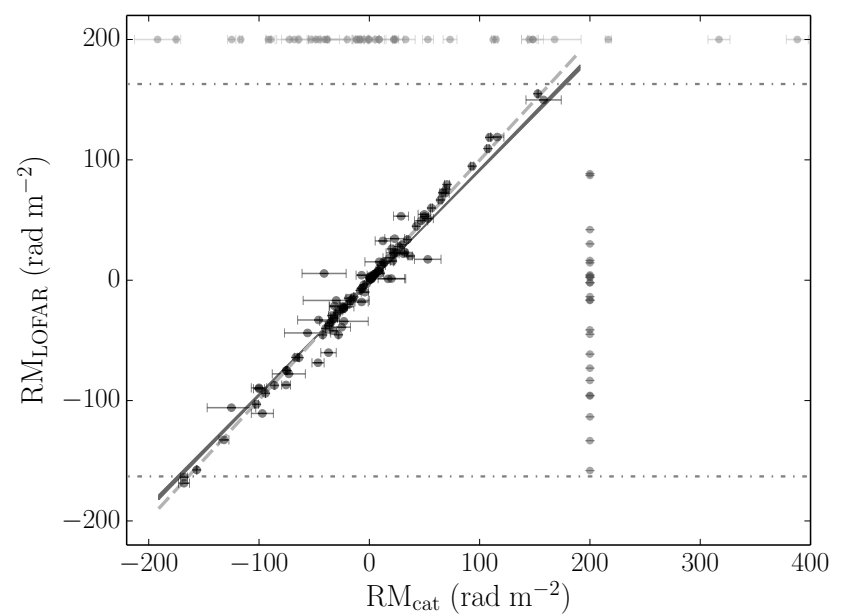

Figure 5. A comparison between the RMs obtained using LOFAR in this work, $\mathrm{RM}_{\mathrm{LOFAR}}$, and the literature, $\mathrm{RM}_{\mathrm{cat}}$, (black points). The expected trend $\mathrm{RM}_{\mathrm{Cat}}=\mathrm{RM}_{\mathrm{LOFAR}}$ is shown by the light grey dashed line. The line of best fit with uncertainties is shown by the dark grey line. The horizontal dash-dotted lines shows the $\left|\phi_{\max }\right|$ at $\left|\mathrm{RM}_{\mathrm{LOFAR}}\right|=163 \mathrm{rad} \mathrm{m}^{-2}$ for the centre frequency $149 \mathrm{MHz}$, see Table 1 . The distribution of RMs obtained in this work without previous measurements are shown by the dark grey points at a constant $\mathrm{RM}_{\mathrm{cat}}=200 \mathrm{rad} \mathrm{m}^{-2}$. The distribution of RMs not detected in this work, but with literature measurements are shown by the light grey points at $\mathrm{RM}_{\mathrm{LOFAR}}=200 \mathrm{rad} \mathrm{m}^{-2}$.

\subsection{Comparison of results with literature measurements}

We verified the RM results we obtained via a comparison with the RMs available in the literature (for 111 pulsars), see Figure 5. The comparison shows that there is very good agreement between the RM measurements. The line of best fit gradient is very close to the expected value of $1(0.94 \pm 0.01$; or $0.99 \pm 0.02$ for an unweighted fit $)$. Furthermore, the majority of measurements (for 102 pulsars) agree within 4 times the published uncertainties. This indicates that the RM uncertainties from the literature are likely generally underestimated. In the few cases with larger discrepancies, either the pulsar catalogue RM had no uncertainty reported (and listed as $0 \mathrm{rad} \mathrm{m}^{-2}$ ), or both measurements have very small uncertainties and may have underestimated possible systematics due to, e.g., the ionospheric $\mathrm{RM}$ correction. This comparison of the RM measurements demonstrates that the RMs measured at low frequencies do not appear to show any disparity compared to those measured at higher frequencies. This confirms that there is no frequency-dependence in RM measurements and that the pulsar magnetosphere does not contribute to the observed RM, to the level of uncertainty to which we can currently measure, as we expected (e.g. Wang et al. 2011).

Furthermore, some independent RM measurements using LOFAR observations were published by Noutsos et al. (2015), 12 of which overlap with this work, see Table A2. Comparing the LOFAR RMs from Noutsos et al. (2015) and this work, the measurements generally agree within the uncertainties; there is a range in agreement from 0.02-2-times the uncertainties. This indicates that we derive reasonable uncertainties on the RMs by adding (in quadrature) the formal uncertainty on the RM measurement from the FDFs and the uncertainty on the $\mathrm{RM}_{\text {ion }}$ corrections.

The precision of the RM (and DM) measurements obtained using the low-frequency LOFAR data are illustrated in Figures 5 and 6. For the 111 pulsars with RMs in both the literature and in this work, the mean uncertainty from the pulsar catalogue and this work are $3.4 \mathrm{rad} \mathrm{m}^{-2}$ and $0.1 \mathrm{rad} \mathrm{m}^{-2}$, respectively. The median fractional uncertainties on the RM measurements are 6.4 and 0.3 per cent, respectively. This indicates that the RMs measured using the low-frequency data are over 20-times more precise (on average) than literature measurements.

Currently, the most substantial contribution to the cumulative uncertainty on the low-frequency RM measurements is the ionospheric RM correction. The median uncertainty on the $\mathrm{RM}_{\mathrm{obs}}$, before ionospheric RM subtraction, is $0.02 \mathrm{rad} \mathrm{m}^{-2}$. The median uncertainty for the $\mathrm{RM}_{\text {ion }}$ estimates is $0.08 \mathrm{rad} \mathrm{m}^{-2}$. If future ionospheric corrections can be improved to the relative precision of the observed RM measurements, the uncertainties in the RM measurements can be improved by an order of magnitude. This will facilitate, for example, precise monitoring of RMs over time to measure and characterise small-scale magneto-ionic structures in the ISM.

Figure 6 shows the DM and modulus RM values towards the pulsars observed in this work, as well as from the pulsar catalogue, for $\mathrm{DM}<250 \mathrm{pc} \mathrm{cm}^{-3}$ and $|\mathrm{RM}|<204 \mathrm{rad} \mathrm{m}^{-2}$. Pulsars located towards low, medium, and high Galactic latitudes are identified using red, orange, and yellow colours, respectively. All of the pulsars with measured RMs from LOFAR are shown, along with the pulsars with literature measurements (64 per cent of the pulsar catalogue within the DM and RM ranges). Also shown are stacked histograms for the DMs and modulus RMs for all of the pulsars in the scatter plots. The distributions approximate log-normal and power-law distributions, respectively. Pulsars towards higher Galactic latitudes tend to have lower DM and RM values, but are still distributed across a range in Galactic magnetic field strengths $(\lessgtr \pm 4 \mu \mathrm{G})$, similar to the mid and low ranges $(\lessgtr \pm 6 \mu \mathrm{G})$.

Figure 7 shows a summary of the current picture of the Faraday sky, along with fractional uncertainties. This includes the allsky Galactic signal reconstructed from 41,632 RMs measured towards extragalactic sources (Oppermann et al. 2015); the 1133 RMs from the current pulsar catalogue (version 1.59; Manchester et al. 2005); and the 137 RM measurements (136 from the pulsar censuses, plus PSR B0329+54) obtained in this work. The large extragalactic catalogues provide densely-packed information about the LoS through the entire Milky Way across the majority of the sky (although measurements are more sparse in the southern sky), however mostly with larger fractional measurement uncertainties. Ongoing work with low-frequency surveys promises to reduce the fractional uncertainties in the extragalactic RM catalogue (e.g. Van Eck et al. 2018; Riseley et al. 2018). While future work using SKA pathfinder surveys promises to increase the number of extragalactic polarised sources further, e.g., MIGHTEE (MeerKAT International GHz Tiered Extragalactic Exploration Survey; Jarvis et al. 2016), POSSUM $^{12}$ (Polarisation Sky Survey of the Universe's Magnetism, using ASKAP), and VLASS ${ }^{13}$ (VLA Sky Survey).

Complementary to the extragalactic information, the pulsar catalogue has fewer pulsars and, therefore, LoSs with RM measurements, the vast majority of which lie within a few degrees of the Galactic disc. However, the majority of these have much lower fractional uncertainties. Figure 7 also demonstrates the trend that (modulus) RMs decrease with increasing (absolute) Galactic latitude, also shown in Figure 6. A result of this is that the fractional uncertainties in the pulsar catalogue RMs (measured at higher observing frequencies) tend to increase with absolute Galactic latitude. The LOFAR measurements provide precise RM data towards pul-

12 http://www.dunlap.utoronto.ca/ askap.org/possum

$13 \mathrm{https} / / / \mathrm{science.nrao.edu/science/surveys/vlass/vlass}$ 

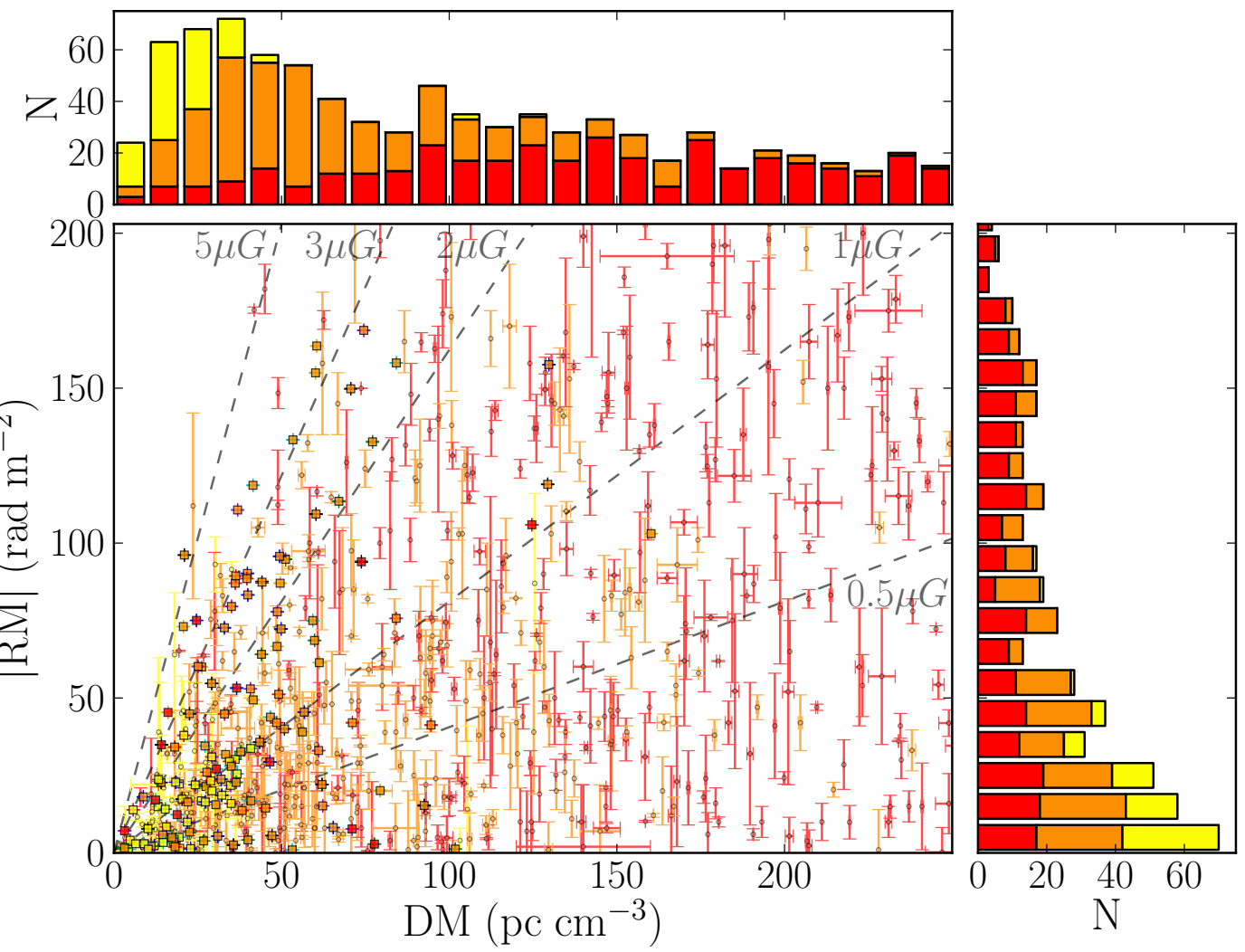

Figure 6. Modulus RM values plotted against DMs for the pulsars with measurements from LOFAR (squares) and the pulsar catalogue (points). The colours represent pulsars at different Galactic latitudes $(b)$ above and below the Galactic plane: $|b|<5$ degrees (red); $5 \leqslant|b|<30$ degrees (orange); and $|b| \geqslant 30$ degrees (yellow). Lines of constant $\left|\left\langle B_{\|}\right\rangle\right|$are shown by the grey dashed lines and corresponding labels. The stacked histograms above/right show the corresponding distribution of all DM and $|\mathrm{RM}|$ measurements (with identical usage of colour).

sars located at a range of Galactic latitudes (above and below the plane, visible from the northern hemisphere) and show consistently low percentage uncertainties. This illustrates that the precision attainable using low-frequency observations is especially valuable for measurements where the absolute RM value is expected to be small, e.g., towards sources that are nearby or at larger absolute Galactic latitudes.

The median pulsar catalogue RM of the pulsars for which we did not detect a significant RM in the LOFAR data is $52.8 \mathrm{rad}$ $\mathrm{m}^{-2}$, with median absolute Galactic latitude and longitude 9.6 and 54.1 degrees, respectively. These pulsars are more likely to be in directions that tend to impart larger RMs, e.g., in the directions of the Galactic plane, the Galactic centre, the North Polar Spur and the boundary of Radio Loop I (large structures identified in the diffuse Galactic radio emission; e.g. Berkhuijsen et al. 1971; Dickinson 2018). Two pulsars in the pulsar census are located towards the plane and Radio Loop I and have catalogued RMs greater than the maximum observable RM attainable for the LOFAR data used (PSR J1859+1526, RM=317 $\pm 10 \mathrm{rad} \mathrm{m}^{-2}$; PSR J1906+1854, $\mathrm{RM}=388 \pm 10 \mathrm{rad} \mathrm{m}^{-2}$ ). For comparison, the median absolute Galactic latitude and longitude of the pulsars that have a significant LOFAR RM are 14.9 and 94.2 degrees, respectively. Therefore, for pulsars in the direction of the Galactic plane and at more considerable distances, increasingly higher frequency observations are complementary to the lower frequency data to enable us to probe the Galactic volume in its entirety. Pulsars in these areas of the Galaxy will also be increasingly difficult to detect at low frequencies due to more substantial dispersion smearing and/or scattering due to the intervening thermal electrons in the ISM.

A reconstruction of the all-sky Galactic RM signal using extragalactic sources (from Oppermann et al. 2015), shown as the background in Figure 7, allows us to compare this information about the entire LoS through our Galaxy to the RMs towards pulsars (located at various distances from the Sun). This indicates how much of the total Faraday depth along each LoS is probed by the foreground pulsars. If there are no large-scale magnetic field reversals in the Galactic halo, then we expect the RMs towards pulsars at greater distances to approach the RM values towards the extragalactic sources. Figure 8 shows the pulsar RMs (from the literature and this work; RMPSR) compared to the same LoS directions from the all-sky Galactic RM map ( $\mathrm{RM}_{\mathrm{EGS}}$ ) for all pulsar LoS located towards Galactic latitudes $|b|>5$ degrees (473 points), coloured according to the pulsar's distance estimates. Although the points seem to be somewhat scattered, 77 per cent of the RMPSR and RM $\mathrm{RGS}_{\mathrm{P}}$ have the same sign. Making cuts for pulsars at lower Galactic latitudes (e.g. $|b|>2$ degrees) reduces this proportion (74 per cent), since closer to the plane there are more likely to be large-scale field reversals and small-scale structures associated with turbulence in the ISM. Making cuts for pulsars at greater Galactic latitudes (e.g. $|b|>8$ degrees) increases this proportion (79 per cent).

We further divided the points in Figure 8 into three distance bins based on the pulsar distance estimates: 'close', 'medium', and 'distant', divided by the 33rd and 66th percentiles of the distances ( $d \leqslant 1.5 \mathrm{kpc}, 1.5<\mathrm{d} \leqslant 3.5 \mathrm{kpc}$, and $\mathrm{d}>3.5 \mathrm{kpc}$, respectively). The cor- 

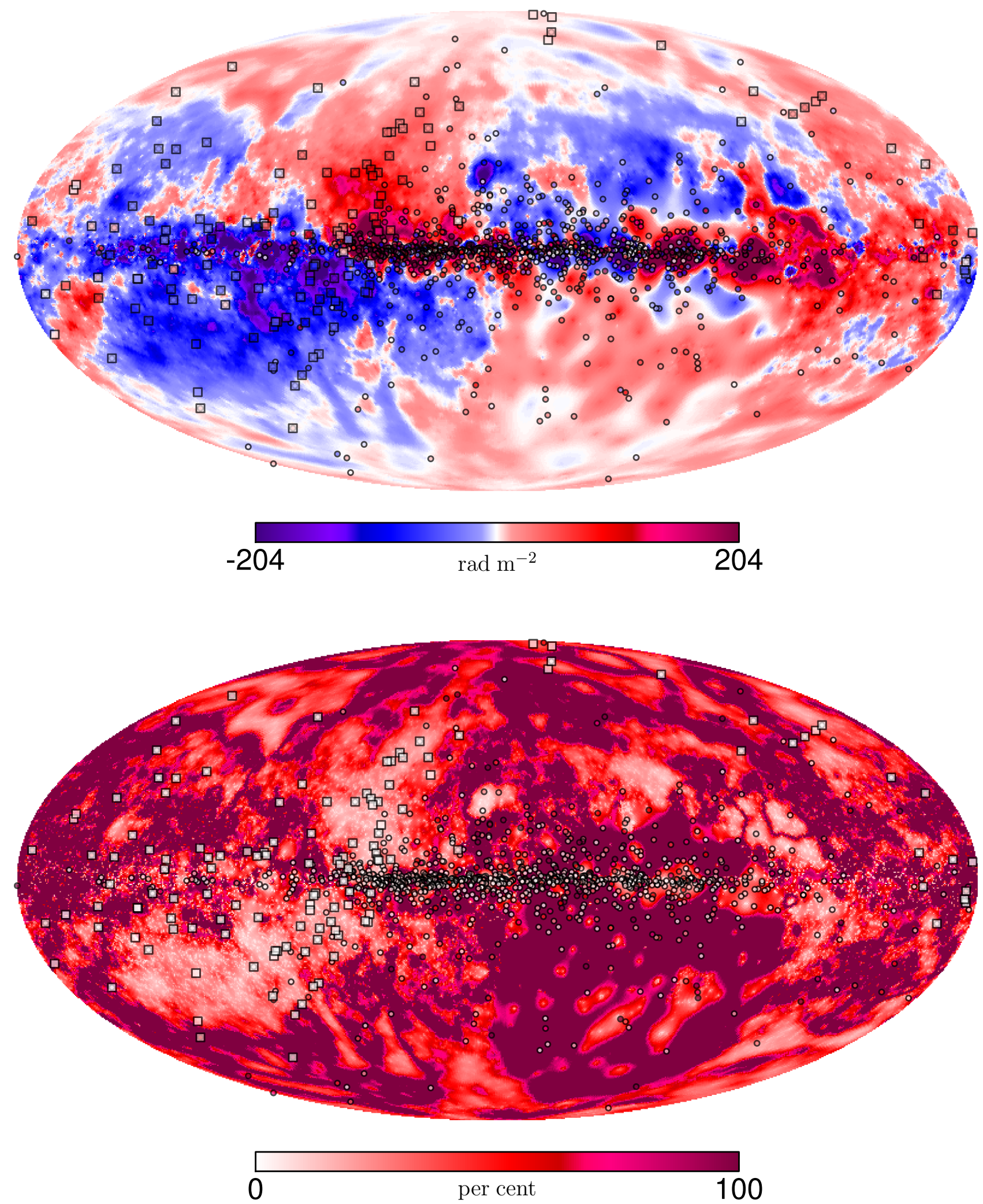

Figure 7. Upper: The RM sky shown in Galactic coordinates using Mollweide projection with $\ell, b=0$ degrees at the centre. The background shows the all-sky Galactic RM signal reconstructed using extragalactic sources. The points show the 1133 RMs from the pulsar catalogue. The squares show the 137 RMs from this work using LOFAR. All data are plotted using the same colour scale, shown by the colour bar in rad $\mathrm{m}^{-2}$, saturated at a cut-off of \pm 204 rad $\mathrm{m}^{-2}$ to emphasise the RM range of the LOFAR data. Positive RMs (reds-pinks) show where the net GMF direction is towards the Earth, and negative RMs (blues-purples) show where the net GMF direction is away from the Earth. Lower: Percentage uncertainties corresponding to the measurements in the upper plot, with corresponding markers. All uncertainties are shown using the same colour scale, shown by the colour bar in per cent and truncated at 100 per cent measurement uncertainty. 


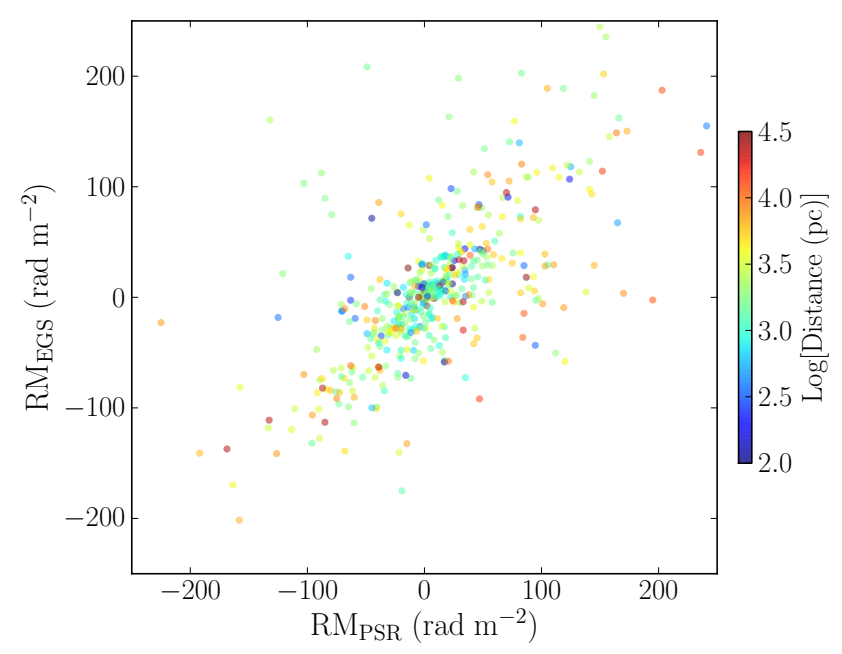

Figure 8. A comparison between the RM towards the pulsars located at $|b|>5$ degrees, $\mathrm{RM}_{\mathrm{PSR}}$, and the Galactic RM signal reconstructed using extragalactic sources, $\mathrm{RM}_{\mathrm{EGS}}$, towards the corresponding LoSs. The points are coloured according to each pulsars' distance estimate in parsecs, shown by the colour bar.

relation coefficient (r-value) between $\mathrm{RM}_{\mathrm{PSR}}$ and $\mathrm{RM} \mathrm{EGS}_{\mathrm{ES}}$ is positive for all distance bins. When reducing the sample to all RMs with the same signs, the correlation coefficients are: $0.76,0.85$, and 0.78 , respectively. Again, making cuts for pulsars at lower latitudes (e.g. $|b| \geqslant 3 \mathrm{deg}$ ), the r-values decrease, except for the pulsars in the 'distant' bin. Making cuts for pulsars at greater latitudes (e.g. $|b| \geqslant 10 \mathrm{deg}$ ), the r-values increase in all bins and are 0.9 for the 'medium', and 'distant' bins. This indicates that RM $\mathrm{PSR}_{\mathrm{P}}$ and RM $\mathrm{MGS}_{\mathrm{EG}}$ tend to become more correlated for pulsars at greater distances, as expected if there are no large-scale field reversals in the Galactic halo magnetic field. However, the variables are perhaps not as well correlated as expected. This may be because at more considerable distances, the distance estimates towards pulsars become more uncertain, see Section 4.5 for further discussion. The differences in the correlation coefficients for different cuts in $|b|$ also indicates that the RMs towards pulsars may have varying contributions from small-scale magneto-ionic foreground structures, for example, supernova remnants or HII regions (e.g. Mitra et al. 2003), which are usually more numerous closer to the Galactic plane compared to higher latitudes, also discussed in Section 4.3. This highlights the requirement for collecting a large set of pulsar RMs for the purpose of studying the large-scale GMF, so that the contributions from small-scale ISM structures may be identified and down-weighted or averaged out in reconstructions of the large-scale GMF structure.

\subsection{Notes on individual pulsars}

The RM measurements towards the Crab Pulsar B0531+21 (located within the Crab Nebula supernova remnant) show a discrepancy of 5.4-times the uncertainties: the pulsar catalogue value measured over 40 years ago is $\mathrm{RM}_{\mathrm{cat}}=-42.3 \pm 0.5 \mathrm{rad} \mathrm{m}^{-2}$ (Manchester 1972) and the RM value measured in this work is $-45.44 \pm 0.08 \mathrm{rad} \mathrm{m}^{-2}$. It is possible that the difference in RM may be because of variations between the observing epochs. Unfortunately, there are very few published RM measurements towards the Crab pulsar for epochs besides these. However, there are monthly updates for other Crab ephemeris parameters ${ }^{14}$, including for the DM (Lyne et al. 1993). Between 1988 May to 2018 September, the minimum and maximum DMs measured were $56.734 \mathrm{pc} \mathrm{cm}^{-3}$ and $56.921 \mathrm{pc} \mathrm{cm}^{-3}$, respectively. The median DM measured during this time period is $56.7805 \mathrm{pc} \mathrm{cm}^{-3}$, which is closer to the value from the LOFAR measurement on 2014 February 15 of $56.7712 \mathrm{pc} \mathrm{cm}^{-3}$. Assuming that the magnetic field value $\left\langle B_{\|}\right\rangle$stays constant, see Table A1, the maximum DM measured would increase the RM by $0.12 \mathrm{rad} \mathrm{m}^{-2}$. This does not account for the $3.1 \mathrm{rad} \mathrm{m}^{-2}$ difference between the RM measurements, indicating that the magnetic field value may also vary along with the electron density.

Such a variation in RM with time has also been observed for the Vela Pulsar B0833-45, located in the Vela Supernova Remnant (e.g. Johnston et al. 2005; Lenc et al. 2017). In these cases, there seem to be small fractional changes in the RMs, with no change in the sign of the RM. Therefore, it is likely that we are sensing both the coherent large-scale Galactic magnetic field component, plus some contribution from a random small-scale foreground magnetic field component, e.g., within the associated supernova remnant structure. In addition, the RM dispersion measured towards the Crab Pulsar, $\sigma_{\mathrm{RM}}<0.25 \mathrm{rad} \mathrm{m}^{-2}$, is above the 92nd percentile of $\sigma_{\mathrm{RM}}$ measurements in this work. This may also suggest some random variations in the magnetic field along the LoS probed by different propagation paths due to scattering, but again, this can be further investigated in more detail using the polarisation profiles in future work. Repetition of observations to monitor these RM variations over long, years, timescales could allow us to estimate the strength and variance of the random magnetic field components associated with the smallscale foreground structure, and deduce the ratio between this and the large-scale, coherent component.

PSR J1810+1744 is an eclipsing black widow pulsar (it is in a short orbital period binary system with a low-mass companion star; e.g., Breton et al. 2013). The RM towards this source has not been published in the literature. In this work, we measured the RM, $88.5 \pm 0.1 \mathrm{rad} \mathrm{m}^{-2}$, using a 20 -minute observation with a total intensity $\mathrm{S} / \mathrm{N}$ of 6 , see Table A1. Shorter integration times further reduce the $\mathrm{S} / \mathrm{N}$, making investigating changes in RM on short timescales over the binary period difficult, as has previously been studied for DM and scattering parameters (Polzin et al. 2018). For the single-epoch observation used in this work, the RM dispersion measured shows that the source is Faraday thin: $<0.0004 \mathrm{rad} \mathrm{m}^{-2}$.

Another black widow pulsar that was observed as part of the LOFAR MSP census is PSR J2051-0827. This pulsar is also subject to repeated timing observations using LOFAR, providing data for multiple different phases of the binary period. The polarisation profile and RM results will be presented in future work (Polzin et al., in prep.).

Some of the pulsars observed in this work are known to change emission modes, e.g. PSR B0823+26 (Sobey et al. 2015; Hermsen et al. 2018), and PSR B0943+10 (Hermsen et al. 2013; Bilous et al. 2014). These pulsars were in the 'bright' emission mode during the observations used to measure the RMs for this work. PSRs $\mathrm{B} 0823+26$ and B0943+10 are among the timing set that are repeatedly observed by LOFAR, and a possible correlation between RM and intrinsic emission mode (although not necessarily expected) will be explored in future work.

\footnotetext{
14 http://www.jb.man.ac.uk/pulsar/crab.html
} 


\subsection{Scale height of the Galactic halo magnetic field}

Although there are fewer RM measurements towards pulsars compared to the extragalactic catalogue (e.g. Oppermann et al. 2015), the additional DM information provided by pulsars allows us to infer the electron-density-weighted average magnetic field parallel towards each LoS. The pulsars are distributed throughout the Galaxy at a range of heights $(\mathrm{Z})$ above/below the Galactic disc, obtained using the YMW16 Galactic electron density model. To demonstrate this, Figure 9 shows the locations of the pulsars in the Galaxy on a 3-D plot in cartesian coordinates. The Sun is located at $(\mathrm{X}, \mathrm{Y}, \mathrm{Z})=(0,8.3,0.006) \mathrm{kpc}$ and the Galactic Centre is at $(\mathrm{X}, \mathrm{Y}, \mathrm{Z})=(0,0,0) \mathrm{kpc}$, as in YMW16, and the Galactic quadrants are also labelled. The markers in Figure 9 are coloured according to the inferred magnetic field strength $\left\langle B_{\|}\right\rangle$and direction. The $\mathrm{X}-\mathrm{Z}$ and Y-Z slices are also shown for further clarity. Figure 9 demonstrates that these data can provide a measurement of the scale height of the Galactic magnetic field in the halo.

Figure 10 shows the magnetic field parallel to the $\operatorname{LoS},\left\langle B_{\|}\right\rangle$, calculated for the pulsars in this work, as well as those from the literature, as a function of their vertical distance from the Galactic plane, Z. All pulsars located in Galactic quadrant I between galactic longitude $30 \leqslant \ell \leqslant 90$ degrees are shown as orange points, and all pulsars located in Galactic quadrant II between galactic longitude $90 \leqslant \ell \leqslant 180$ degrees are shown by the violet points. These ranges were chosen to investigate the directions towards the inner Galaxy (excluding regions closer towards the Galactic centre) and the outer Galaxy, respectively. These are also the ranges where the largest number of pulsars with LOFAR data are located, see Figure 9.

Figure 10 shows that for Galactic quadrant I, there is a notable dichotomy between mostly positive (negative) magnetic field values for pulsars located above (below) the plane, similarly evident in Figure 7. Although the large region of positive $\left\langle B_{\|}\right\rangle$seems to be coincident with the North Polar Spur feature (e.g. Sun et al. 2015), this effect may be somewhat related to the large-scale magnetic field reversal in the Galactic plane in this quadrant (e.g. Van Eck et al. 2011). However, the large-scale reversal does not appear to affect the bulk sign of the RM either above or below the Galactic plane and perhaps the effect of the large-scale reversal may not be visible in the halo and confined to the plane. Conversely, in Galactic quadrant II, where a large-scale magnetic field reversal is not expected (e.g. Van Eck et al. 2011), there is a majority of negative magnetic field values towards pulsars located both above and below the plane. Although the pulsar data are more sparse towards the Galactic anticentre, with half of the number of data points compared to quadrant I and 35 per cent more data points below the plane. For comparison, in quadrant I there are approximately equal numbers of points above and below the plane. The median magnetic field strengths and directions in Galactic quadrants I and II using these data are 0.76 and $-0.77 \mu \mathrm{G}$, respectively. These values are consistent with the strength of the regular halo magnetic field of $2 \mu \mathrm{G}$ or lower from Sun \& Reich (2010).

We use the data shown in Figure 10 to fit for the magnetic scale height in the Galactic halo, using the form:

$\left\langle B_{\|}\right\rangle=\left\langle B_{\|, 0}\right\rangle \exp (-\mathrm{Z} / \mathrm{H})$,

where $\left\langle B_{\|, 0}\right\rangle$ is the largest value of the magnetic field at $\mathrm{Z}=0$, and $\mathrm{H}$ is the magnetic scale height. Both $\left\langle B_{\|}\right\rangle$and $\mathrm{Z}$ can take positive and negative values depending on the areas identified in Figure 10. Since the RM data provide the magnetic field strength and net direction parallel to the LoS, these will provide a lower limit for the scale height of the total GMF. Therefore, we fit the 'outermost' points
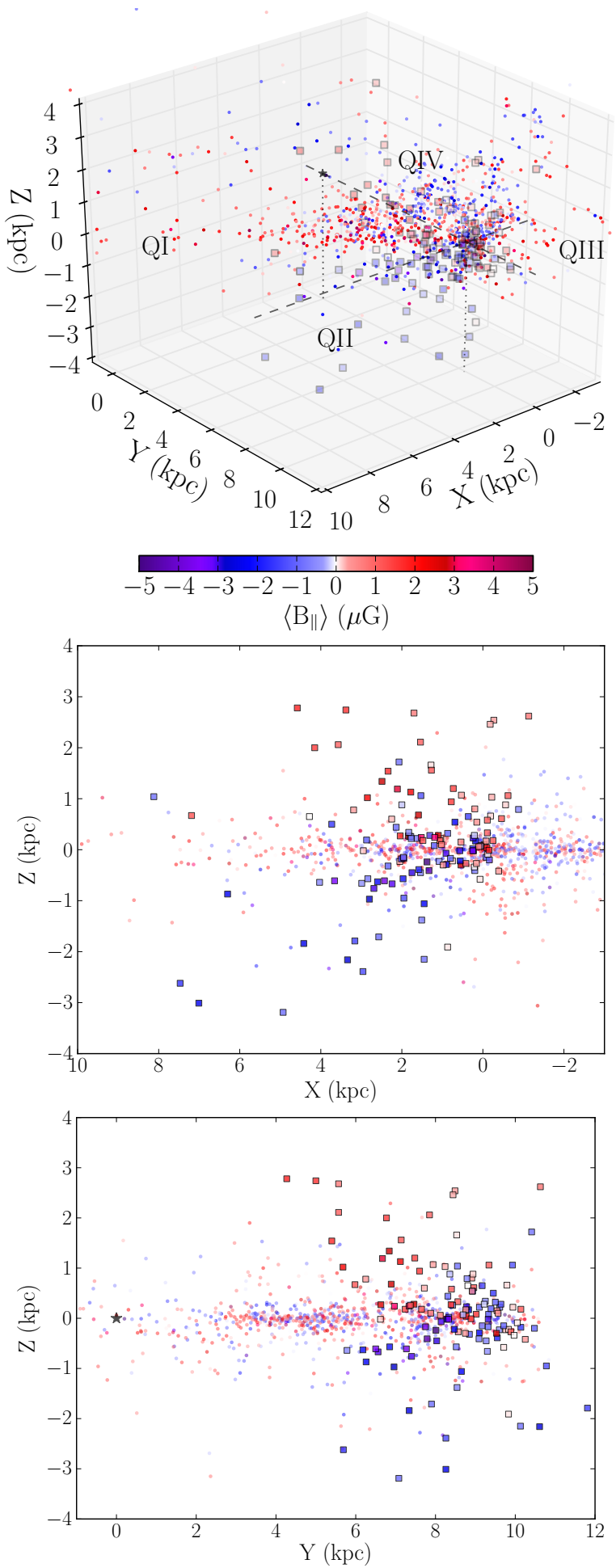

Figure 9. Upper: 3-D plot showing the location of pulsars, in Galactocentric coordinates, with colours representing $\left\langle B_{\|}\right\rangle$in $\mu \mathrm{G}$, see colour bar. The points show the pulsars with DM and RM measurements from the literature. The squares show the pulsars with DM and RM measurements from this work using LOFAR. The location of the Galactic Centre is shown by the black star, with the black dotted line connecting it to the $x-y$ axis. The Galactic quadrants are labelled QI-IV, and separated by the dashed lines. The intersection of the lines show the location of the Sun, which is also connected to the $x-y$ plane by the grey dotted line. Middle, Lower: The same points from the upper plot are shown in the X-Z, and Y-Z planes, respectively. 18 pulsars (10 with literature RMs and 8 with LOFAR RMs) have larger $\mathrm{Z}$ values $(>8 \mathrm{kpc})$ and are not shown here. 


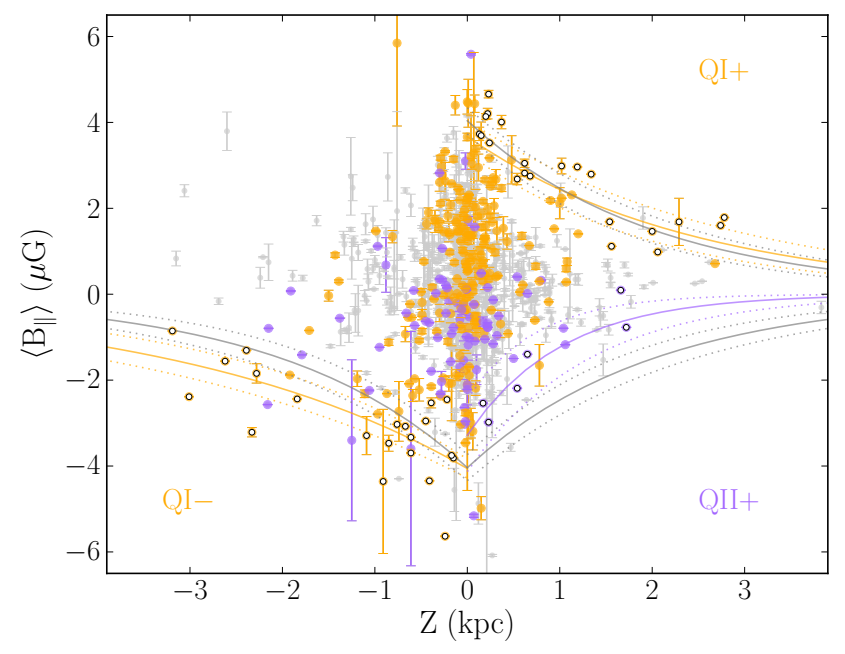

Figure 10. Using the RM and DM measurements from the pulsar catalogue and this work, $\left\langle B_{\|}\right\rangle$is shown against the estimated height above/below the Galactic plane, Z, (grey points). The pulsars located in Galactic quadrant I in the range $30 \leqslant \ell<90$ degrees are shown by the orange points, and pulsars located in Galactic quadrant II in the range $90<\ell<180$ degrees are shown using the violet points. Three areas in the plot are identified: Galactic quadrant I, above the plane, with positive $\left\langle\boldsymbol{B}_{\|}\right\rangle$values (labelled QI+ in orange); Galactic quadrant I, below the plane, with negative $\left\langle B_{\|}\right\rangle$ values (labelled QI- in orange); and Galactic quadrant II, above the plane, with negative $\left\langle B_{\|}\right\rangle$values (labelled QII+ in violet). The 'outermost' points identified (see text) in these areas are shown by the open circles. Fits of the magnetic scale height to the 'outermost' points using Equation 5 (solid lines) and the uncertainties (dotted lines) are shown for QI+ and QI- (orange lines) and QII+ (violet lines). We also show the fit for the magnetic scale height to all of the absolute values of the outermost points (grey solid line) and the uncertainties (grey dotted lines) for comparison.

for pulsars in: Galactic quadrant I above the plane where $\left\langle B_{\|}\right\rangle>0$ (labelled QI+); Galactic quadrant I below the plane where $\left\langle B_{\|}\right\rangle<0$ (labelled QI-); and in Galactic quadrant II above the plane where $\left\langle B_{\|}\right\rangle<0$ (labelled QII+), see Figure 10. The 'outermost' points were identified as the largest absolute values of $\left\langle B_{\|}\right\rangle$in the bin ranges $0.1<|Z| \leqslant 0.5 \mathrm{kpc}, 0.5<|\mathrm{Z}| \leqslant 1.5 \mathrm{kpc}$, and $1.5<|\mathrm{Z}| \leqslant 3.5 \mathrm{kpc}$. For the areas QI+ and QI-, the number of points selected in each $\mathrm{Z}$ bin was chosen to be one fewer than the total number of data points in the bin with the fewest number, i.e., in the bin $1.5<|\mathrm{Z}| \leqslant 3.5 \mathrm{kpc}$. For the area QII+ with fewer data points, the number of points in the $1.5<|Z| \leqslant 3.5 \mathrm{kpc}$ bin is 2 , and so 2 points were also selected from the $0.1<|Z| \leqslant 0.5 \mathrm{kpc}$ and $0.5<|Z| \leqslant 1.5 \mathrm{kpc}$ bins. Pulsars in Galactic quadrant II below the plane where $\left\langle B_{\|}\right\rangle<0$ were not fit as there are insufficient data. The 'outermost' data points fit in each area are shown as open circles in Figure 10. Table 3 summarises the parameters obtained from the magnetic scale height fit using Equation 5. We also fit the scale height for the absolute values of all of the points fit in the individual areas, shown by the grey lines in Figure 10 and included in Table 3.

Table 3 shows that the general magnetic scale height found using the data for all areas identified in Figure 10 is $2.0 \pm 0.3 \mathrm{kpc}$. The mean of the magnetic scale heights for the identified areas is $2.2 \pm 0.5 \mathrm{kpc}$. All of the scale heights presented in Table 3 agree within the uncertainties, except for the QII+ area. This indicates that the scale height in Galactic quadrant I towards the inner Galaxy is larger than the scale height in Galactic quadrant II towards the outer Galaxy. Only six data points were fit for the QII+ area, and so a larger sample would be necessary to provide a more confident outcome for
Table 3. Summary of the parameters obtained from fitting the magnetic scale height function in Equation 5. Uncertainties ( \pm ) are quoted in the columns to the right of the values.

\begin{tabular}{|c|c|c|c|c|}
\hline Quadrant & $\begin{array}{l}\text { Magnetic scale height }(\mathrm{H}) \\
\mathrm{kpc}\end{array}$ & $\begin{array}{l} \pm \\
\mathrm{kpc}\end{array}$ & $\begin{array}{l}\left\langle B_{\|, 0}\right\rangle \\
\mu \mathrm{G}\end{array}$ & $\begin{array}{l} \pm \\
\mu G\end{array}$ \\
\hline QI+ & 2.4 & 0.4 & 3.7 & 0.4 \\
\hline QI- & 3.3 & 0.6 & 4.0 & 0.2 \\
\hline QII+ & 1.0 & 0.3 & 3.3 & 0.8 \\
\hline All & 2.0 & 0.3 & 4.0 & 0.3 \\
\hline
\end{tabular}

the direction of the outer Galaxy. All of the $\left\langle B_{\|, 0}\right\rangle$ values in Table 3 agree within the uncertainties, and the value for all areas is $4 \mu \mathrm{G}$. We extrapolated the electron-density-weighted average magnetic field to a greater distance from the galactic plane $( \pm 6 \mathrm{kpc})$ using the results summarised in Table 3. At this height, the $\left\langle B_{\|, 0}\right\rangle$ in areas QI+, QI-, QII+, and 'All' are $0.3 \pm 0.1 \mu \mathrm{G},-0.6 \pm 0.2 \mu \mathrm{G},-0.01 \pm 0.02 \mu \mathrm{G}$, and $0.2 \pm 0.1 \mu \mathrm{G}$, respectively.

The uncertainties from the fits are shown in Figure 10 and Table 3, and account for the uncertainties in the measurement of $\left\langle B_{\|}\right\rangle$, but not in $\mathrm{Z}$, which are generally not known for pulsars with distance estimates but may have larger fractional uncertainties than the uncertainties on $\left\langle B_{\|}\right\rangle$. There are 5 and 3 data points in QI+ and QI-, respectively, that were not included in the fits because their $|Z|$ distance estimates are much larger than the general population $(>8 \mathrm{kpc})$ and likely overestimated. The small uncertainties on $\left\langle B_{\|}\right\rangle$ from the LOFAR data at high $|Z|$ values tend to flatten the fits (especially in QI-), increasing the scale height and decreasing the $\left\langle B_{\|, 0}\right\rangle$ output. Therefore, higher numbers of pulsar data points with smaller uncertainties, especially at high $|Z|$ values, are desirable for increasing the accuracy of determining the magnetic scale height(s) in future. Also valuable towards this goal are independent distance estimates for these pulsars, see Section 4.5 for further discussion.

The magnetic scale height summarised in Table 3 is comparable, although generally somewhat larger than, the Galactic free electron vertical scale heights determined using pulsar DMs, e.g. $1.8_{-0.3}^{+0.1} \mathrm{kpc}$ from Gaensler et al. (2008); $1.4_{-0.2}^{+0.3} \mathrm{kpc}$ from Savage \& Wakker (2009); $1.6 \pm 0.3 \mathrm{kpc}$ from Schnitzeler (2012); or $1.67 \pm 0.05 \mathrm{kpc}$ from YMW16. This corresponds to the thick disc of the Galaxy, which has a more extensive scale height than the thin disk with scale heights between $20-70$ pc (e.g. Yao et al. 2017, and references therein). Since the Faraday rotation effect is caused by both the electron density and the magnetic field in the ISM, the scale heights obtained using RMs will not be independent of the electron density scale height. This may be why the scale height found here is also larger than the $0.74 \mathrm{kpc}$ exponential scale height of synchrotron emission found in, e.g., Sun \& Reich (2012). Although, in this case, synchrotron modelling also requires ancillary parameters, such as the relativistic electron population, and is also sensitive to the magnetic field perpendicular to the LoS. This provides an incentive to use at least one magnetic field observable to derive the properties and structure of the total GMF, including the anisotropy of the Galactic halo magnetic field, see Section 4.5. There are complex analytical models for large-scale Galactic halo magnetic fields (e.g. Ferrière \& Terral 2014). Which of these models best fit the RM data will be explored in future work.

The scale heights determined for the Milky Way can also be compared to external galaxies. For example, for the edge-on galaxies NGC891 and NGC4631, Hummel et al. (1991) deduced scale heights of $0.9 \mathrm{kpc}$ and $1.3 \mathrm{kpc}$, respectively, using the increase in the mean degree of polarisation with distance from the galactic disk. The numbers obtained here are also comparable or up to three times 
larger than these edge-on galaxies. The scale heights estimated here are also approximately equivalent to or greater than the radio scale heights seen in other galaxies (e.g. Krause et al. 2018).

\subsection{Limitations of current data and future prospects}

In future, polarisation calibration will be performed on the data described in this work, to present the low-frequency polarisation profiles. This may increase the $\mathrm{S} / \mathrm{N}$ in linear polarisation for some pulsars so that an RM may be detected. In the cases where the linearly polarised $\mathrm{S} / \mathrm{N}$ was less than the threshold set (i.e. $\mathrm{S} / \mathrm{N}_{F}<4$ ), it would be preferable to observe these sources for longer integration times to obtain more reliable detections and decrease the uncertainties. For pulsars that are being repeatedly observed in LOFAR timing campaigns (this includes a subset of both MSPs and 'slow' pulsars) the $\mathrm{S} / \mathrm{N}$ in the polarisation profiles can also be increased by concatenating observations. However, ionospheric RM corrections will have to be made before the addition in this case. For this set of pulsars with higher $\mathrm{S} / \mathrm{N}$ detections, the repeated observations can also be used to better constrain the RM (and uncertainty) after correcting for the ionospheric RM for several independent epochs, as was done as an example in this work for PSR B0329+54. The precise measurements now routinely obtained using low-frequency observations ushers us into an era where ISM parameters such as DMs, RMs, and scattering (e.g. Geyer et al. 2017; Michilli et al. 2018a) can be monitored over time, also allowing us to probe small-scale turbulent structures in the ISM and other foregrounds (e.g. Howard et al. 2016; Tiburzi \& Verbiest 2018).

The maximum absolute RM measured in this work is towards PSR B2210+29 $\left(\mathrm{RM}_{\mathrm{obs}}=-165.23 \mathrm{rad} \mathrm{m}^{-2} ; \mathrm{RM}_{\mathrm{ISM}}=-168.66 \mathrm{rad}\right.$ $\mathrm{m}^{-2}$, after the ionospheric correction). Due to the channel widths used for the data set, the largest Faraday depth to which one loses 50 per cent sensitivity, at the centre frequency, is $163 \mathrm{rad} \mathrm{m}^{-2}$ (not accounting for the loss of frequency information due, e.g., to excised RFI). It is possible to use smaller channel widths for LOFAR observations, expanding this largest Faraday depth beyond $1000 \mathrm{rad}$ $\mathrm{m}^{-2}$. These data were not used in this work, as the data volume becomes more substantial and the data reduction more cumbersome. However, for areas where larger RMs are expected (e.g. towards the Galactic plane, at larger distances, and towards the boundary of Radio Loop I), also usually accompanied by larger DMs, the finer channel resolution can be utilised to detect the pulsars and measure larger absolute RMs. Probing the Galaxy in the Galactic plane and at more considerable distances, DMs and RMs measured using higher frequency observations will be complementary to the measurements from low-frequency observations. This is because pulsars with large DMs and RMs may become scattered or depolarised in lower-frequency observations. In this work, we demonstrated that the low-frequency LOFAR observations provide excellent fractional uncertainties on RMs (and DMs) towards pulsars with relatively low absolute RMs, e.g., that are located towards the Galactic halo and are relatively close to the Sun.

More pulsars are being discovered in ongoing pulsar surveys at various centre frequencies, including at low radio frequencies using LOFAR (e.g. Coenen et al. 2014; Michilli et al. 2018b; Tan et al. 2018). The pulsars discovered can be followed up with polarisation observations to obtain RM measurements, to increase the numbers of LoSs with which we can probe the structure of the Galaxy. The low-frequency discoveries are valuable because they are located relatively nearby or towards the Galactic halo (e.g. van Leeuwen \& Stappers 2010). This provides local GMF estimates, which can be compared to the data from more distant pulsars or extragalactic sources for longer path lengths through the Galaxy. Moreover, pulsars at larger distances and in the Galactic plane are preferentially discovered at higher frequencies using other large radio telescopes (e.g. Stovall et al. 2014; Ng et al. 2014; Lyne et al. 2017), and are useful for mapping the GMF towards the more distant areas of our Galaxy. More known pulsars that are distributed throughout the Galaxy at various distances from the Sun provide more data with which to reconstruct the GMF structure (including any field reversals along the LoSs) with greater confidence. In the future, the observational capabilities of the SKA to discover and time pulsars will allow us to approximately triple the number of known pulsars in the Galaxy (e.g. Keane et al. 2015; Xue et al. 2017), as well as measure their parallax and proper motions using VLBI (e.g. Paragi et al. 2015), enabling 3-D tomography of the electron density and GMF (e.g. Han et al. 2015).

In order to reconstruct an accurate model of the GMF (and electron density) using RMs and DMs towards pulsars, it is becoming increasingly necessary to determine independent distance measurements towards larger numbers of pulsars. For the set of pulsars with RMs measured in this work, 17 slow pulsars and 13 MSPs (15 and 68 per cent of the set, respectively) have one (or more) independent distance measurements (see Yao et al. 2017, and references therein): via annual parallax using VLBI (14 slow and 5 MSPs, respectively), or timing ( 0 and 6 , respectively); association with a nebula ( 1 and 0 , respectively); kinematic distances from $\mathrm{HI}$ absorption measurements ( 2 and 0 , respectively); or an optical counterpart association ( 0 and 2, respectively) (Jennings et al. 2018). Although pulsar distances can be estimated using a Galactic electron density model, and these are becoming more accurate with more independent distance measurements that can be used to calibrate the models, there are still large numbers of pulsars for which distances are over 40 per cent uncertain (Yao et al. 2017). We used the recently published YMW16 electron density model for this work because previous models had a maximum $|\mathrm{Z}|$ limit of $\leqslant 2 \mathrm{kpc}$. The YMW16 model allows larger heights above the Galactic plane, e.g. shown in Figure 10, facilitating the magnetic scale height fit. There are efforts towards increasing the number of pulsars with independent distance measurements, e.g., Deller et al. (2018) provide an annual parallax for a further 21 pulsars with RMs in this work. For these pulsars, the median discrepancy between the DM distance estimates and the VLBI annual parallax distance measurements is $0.67 \mathrm{kpc}$ (or 54 per cent fractional difference). However, in a couple of extreme cases, the DM distance estimates are overestimated by $\approx 20 \mathrm{kpc}$ for PSRs B2303+30 and B2210+29 towards Galactic coordinates $\ell \approx 90, b \approx-25$. It is essential to continue to obtain independent distance measurements for more pulsars so that 3-D tomography of the Galactic (magnetic field) structure using the precise ISM parameters measured using the SKA and its precursors can be fully realised (e.g. Han et al. 2015).

Studies using RMs alone cannot distinguish between ordered random and isotropic random field components, which are often grouped into the 'random' field component (e.g. Jaffe et al. 2010). Moreover, Equation 1 assumes that the magnetic field and thermal electron density are uncorrelated. However, if their fluctuations are (anti)correlated, this will yield an (under)overestimate of $\left\langle B_{\|}\right\rangle$, and can result in error by a factor of $2-3$ in a statistically homogeneous magneto-ionic medium (Beck et al. 2003). Therefore, combining results from the other magnetic field tracers is desirable, to obtain as complete a picture of the 3-D GMF structure as possible. In the near future it may also be possible to include observations of the directions of arrival of Ultra-High Energy Cosmic Rays, but the sources and composition of these still need to be constrained (e.g. 
Aab et al. 2018, and references therein). The framework required to combine multiple observables, as well as theoretical models, to infer the most likely model of the structure of the Galaxy is being constructed and refined (e.g. IMAGINE; Boulanger et al. 2018). In the future, the SKA will also provide groundbreaking observations for many of the complementary observables of the GMF, particularly with respect to diffuse polarisation, extragalactic RM-grids, and Zeeman splitting (Haverkorn et al. 2015; JohnstonHollitt et al. 2015; Robishaw et al. 2015, respectively), promising to revolutionise our understanding of the GMF.

\section{CONCLUSIONS}

(i) We have measured RMs towards 137 pulsars using the 'LOFAR census of non-recycled pulsars' (Bilous et al. 2016), the 'LOFAR census of millisecond pulsars' (Kondratiev et al. 2016), and repeated timing/monitoring observations of PSR B0329+54. We present the largest low-frequency RM catalogue to date, with 25 pulsars that do not have RMs published in the literature. For the remaining pulsars with previously published measurements, the low-frequency data generally agree with previous measurements, within uncertainties, and provide 20-times greater precision, on average. The RMs were corrected for ionospheric Faraday rotation using the IONFR code, with IGS VTEC maps and the IGRF-12 as inputs. This correction is essential for low-frequency data, where the largest contribution to the uncertainty is the current method for subtracting the ionospheric RM.

(ii) The RMs were measured using the RM-synthesis method and the RM dispersion, $\sigma_{\mathrm{RM}}$, was obtained using the RMCLEAN algorithm. The RM dispersions show that the majority of the pulsars are Faraday thin sources: $<0.001 \mathrm{rad} \mathrm{m}^{-2}$. However, in some cases, e.g., the Crab Pulsar B0531+21, the larger RM dispersion found may be due to small-scale magneto-ionic structure associated with its supernova remnant.

(iii) We used the current RM and DM measurements available from the pulsar catalogue and this work to measure the magnetic scale height of the GMF in the halo, for a range of Galactic longitudes towards Galactic quadrants I and II, where the majority of the LOFAR measurements are located. Fitting all points, we found a general scale height of $2.0 \pm 0.3 \mathrm{kpc}$ - comparable to the scale height of Galactic free electrons published in the literature. Although distance estimates are available for all pulsars, independent distance measurements, e.g., annual parallax measurements, are important for the purpose of reconstructing the magnetic scale height in the halo and the GMF structure in general.

(iv) The RM measurements from this work present an initial precise catalogue, which will be expanded and also increased in accuracy by using the LOFAR timing data towards a set of slow pulsars and MSPs. Precise DMs and RMs from low-frequency instruments, such as these from LOFAR, are becoming routine, promising an era of monitoring for time variability, which can be used to further investigate, for example, small-scale foreground ISM structures.

(v) The results from the low-frequency pathfinder/precursor telescopes show the promise of the SKA. For example, the lowfrequency SKA precursor in the southern hemisphere, the MWA, is also routinely observing pulsars (e.g. Xue et al. 2017). The DM and RM measurements the MWA can provide are complementary to LOFAR, from which an all-sky low-frequency catalogue can be assembled, allowing us to study the 3-D Galactic magnetic field structure in more detail. In the future, the SKA will revolutionise our knowledge of pulsars, magnetism, and our Galaxy through discov- ering, timing, and measuring the astrometric properties of a large number of pulsars in our Galaxy. Efforts towards an RM-grid of extragalactic sources, diffuse Galactic synchrotron emission maps and Zeeman splitting will also supply complementary magnetic field observables to reconstruct a more complete picture of the total GMF structure. Bayesian inference frameworks such as IMAGINE can enable us to combine these observables and theoretical models and to assess the likely (magnetic) structure of our Galaxy.

\section{ACKNOWLEDGEMENTS}

This paper is based on data obtained with the International LOFAR Telescope (ILT) under project codes LC1_003, LC1_027. LOFAR (van Haarlem et al. 2013) is the Low Frequency Array designed and constructed by ASTRON. It has observing, data processing, and data storage facilities in several countries, that are owned by various parties (each with their own funding sources), and that are collectively operated by the ILT foundation under a joint scientific policy. The ILT resources have benefitted from the following recent major funding sources: CNRS-INSU, Observatoire de Paris and Université d'Orléans, France; BMBF, MIWF-NRW, MPG, Germany; Science Foundation Ireland (SFI), Department of Business, Enterprise and Innovation (DBEI), Ireland; NWO, The Netherlands; The Science and Technology Facilities Council, UK. We thank Tom Hassall for his assistance in acquiring the data, and Gregory Desvignes for his assistance with ionospheric RM corrections. We thank Leszek Błaszkiewicz and Dominic Schnitzeler for their useful comments. J.W.T.H., V.I.K., and D.M. acknowledge funding from an NWO Vidi fellowship and ERC Starting Grant "DRAGNET" (337062). The research leading to these results has received funding from the European Research Council under the European Union's Seventh Framework Programme (FP/2007-2013) / ERC Grant Agreement n. 617199. This work used the Python plotting library MatPlotlib (Hunter 2007). This research has made use of NASA's Astrophysics Data System Bibliographic Services. We thank the referee, Kevin Stovall, for providing helpful comments.

\section{REFERENCES}

Aab A., et al., 2018, ApJ, 853, L29

Aharonian F., Bykov A., Parizot E., Ptuskin V., Watson A., 2012, Space Sci. Rev., 166, 97

Anderson C. S., Gaensler B. M., Feain I. J., Franzen T. M. O., 2015, ApJ, 815,49

Beck R., 2001, Space Sci. Rev., 99, 243

Beck R., 2009, in Strassmeier K. G., Kosovichev A. G., Beckman J. E., eds, IAU Symposium Vol. 259, Cosmic Magnetic Fields: From Planets, to Stars and Galaxies. pp 3-14 (arXiv:0812.4925), doi:10.1017/S1743921309030014

Beck R., Shukurov A., Sokoloff D., Wielebinski R., 2003, A\&A, 411, 99

Beck R., et al., 2015, Advancing Astrophysics with the Square Kilometre Array (AASKA14), p. 94

Beck M. C., Beck A. M., Beck R., Dolag K., Strong A. W., Nielaba P., 2016, J. Cosmology Astropart. Phys., 5, 056

Berkhuijsen E. M., Haslam C. G. T., Salter C. J., 1971, A\&A, 14, 252

Bilous A. V., et al., 2014, A\&A, 572, A52

Bilous A. V., et al., 2016, A\&A, 591, A134

Boulanger F., et al., 2018, J. Cosmology Astropart. Phys., 8, 049

Boyles J., et al., 2013, ApJ, 763, 80

Brentjens M. A., de Bruyn A. G., 2005, A\&A, 441, 1217

Breton R. P., et al., 2013, ApJ, 769, 108

Brinkman C., Freire P. C. C., Rankin J., Stovall K., 2018, MNRAS, 474, 2012 
Brown J. C., Taylor A. R., Wielebinski R., Mueller P., 2003, ApJ, 592, L29

Brown J. C., Haverkorn M., Gaensler B. M., Taylor A. R., Bizunok N. S., McClure-Griffiths N. M., Dickey J. M., Green A. J., 2007, ApJ, 663, 258

Burn B. J., 1966, MNRAS, 133, 67

Coenen T., et al., 2014, A\&A, 570, A60

Cordes J. M., Lazio T. J. W., 2002, ArXiv Astrophysics e-prints,

Crutcher R. M., 2012, ARA\&A, 50, 29

Crutcher R. M., Wandelt B., Heiles C., Falgarone E., Troland T. H., 2010, ApJ, 725, 466

Dach R., Schaer S., Arnold D., Prange L., Sidorov D., Stebler P., Villiger A., Jäggi A., 2018, Astronomical Institute, University of Bern,

Dai S., et al., 2015, MNRAS, 449, 3223

Deller A. T., et al., 2018, preprint, (arXiv: 1808.09046)

Dickinson C., 2018, Galaxies, 6, 56

Dobbs C. L., Price D. J., Pettitt A. R., Bate M. R., Tricco T. S., 2016, MNRAS, 461, 4482

Farrar G. R., Jansson R., Feain I. J., Gaensler B. M., 2013, J. Cosmology Astropart. Phys., 1, 23

Ferrière K., 2011, Mem. Soc. Astron. Italiana, 82, 824

Ferrière K., Terral P., 2014, A\&A, 561, A100

Force M. M., Demorest P., Rankin J. M., 2015, MNRAS, 453, 4485

Gaensler B. M., Madsen G. J., Chatterjee S., Mao S. A., 2008, Publ. Astron. Soc. Australia, 25, 184

George S. J., Stil J. M., Keller B. W., 2012, Publ. Astron. Soc. Australia, 29, 214

Geyer M., et al., 2017, MNRAS, 470, 2659

Hall J. S., 1949, Science, 109, 166

Hamilton P. A., Lyne A. G., 1987, MNRAS, 224, 1073

Han J. L., Manchester R. N., Qiao G. J., 1999, MNRAS, 306, 371

Han J. L., Manchester R. N., Lyne A. G., Qiao G. J., van Straten W., 2006, ApJ, 642, 868

Han J., et al., 2015, Advancing Astrophysics with the Square Kilometre Array (AASKA14), p. 41

Han J. L., Manchester R. N., van Straten W., Demorest P., 2018, ApJS, 234, 11

Hankins T. H., Rankin J. M., 2010, AJ, 139, 168

Haverkorn M., 2015, in Lazarian A., de Gouveia Dal Pino E. M., Melioli C., eds, Astrophysics and Space Science Library Vol. 407, Astrophysics and Space Science Library. p. 483 (arXiv : 1406 . 0283), doi:10.1007/9783-662-44625-6_17

Haverkorn M., Katgert P., de Bruyn A. G., 2004, A\&A, 427, 169

Haverkorn M., Gaensler B. M., Brown J. C., Bizunok N. S., McClureGriffiths N. M., Dickey J. M., Green A. J., 2006, ApJ, 637, L33

Haverkorn M., et al., 2015, Advancing Astrophysics with the Square Kilometre Array (AASKA14), p. 96

Heald G., Braun R., Edmonds R., 2009, A\&A, 503, 409

Heald G., McKean J., Pizzo R., eds, 2018, Low Frequency Radio Astronomy and the LOFAR Observatory. Springer International Publishing, doi:10.1007/978-3-319-23434-2

Hermsen W., et al., 2013, Science, 339, 436

Hermsen W., et al., 2018, MNRAS, 480, 3655

Hernández-Pajares M., et al., 2009, Journal of Geodesy, 83, 263

Hiltner W. A., 1949, Science, 109, 165

Hotan A. W., van Straten W., Manchester R. N., 2004, Publ. Astron. Soc. Australia, 21, 302

Howard T. A., Stovall K., Dowell J., Taylor G. B., White S. M., 2016, The Astrophysical Journal, 831, 208

Hummel E., Beck R., Dahlem M., 1991, A\&A, 248, 23

Hunter J. D., 2007, Computing In Science \& Engineering, 9, 90

Jaffe T. R., Leahy J. P., Banday A. J., Leach S. M., Lowe S. R., Wilkinson A., 2010, MNRAS, 401, 1013

Jaffe T. R., Banday A. J., Leahy J. P., Leach S., Strong A. W., 2011, MNRAS, 416,1152

Jansson R., Farrar G. R., 2012, ApJ, 761, L11

Jarvis M., et al., 2016, in Proceedings of MeerKAT Science: On the Pathway to the SKA. 25-27 May, 2016 Stellenbosch,
South Africa (MeerKAT2016). Online at https://pos.sissa.it/cgibin/reader/conf.cgi?confid=277, id.6. p. 6 (arXiv : 1709.01901)

Jelić V., et al., 2015, A\&A, 583, A137

Jennings R. J., Kaplan D. L., Chatterjee S., Cordes J. M., Deller A. T., 2018, ApJ, 864, 26

Johnston S., Kerr M., 2018, MNRAS, 474, 4629

Johnston-Hollitt M., et al., 2015, Advancing Astrophysics with the Square Kilometre Array (AASKA14), p. 92

Johnston S., Hobbs G., Vigeland S., Kramer M., Weisberg J. M., Lyne A. G., 2005, MNRAS, 364, 1397

Johnston S., Kramer M., Karastergiou A., Hobbs G., Ord S., Wallman J., 2007, MNRAS, 381, 1625

Johnston S., Karastergiou A., Mitra D., Gupta Y., 2008, MNRAS, 388, 261

Keane E. F., 2018, in Weltevrede P., Perera B. B. P., Preston L. L., Sanidas S., eds, IAU Symposium Vol. 337, Pulsar Astrophysics the Next Fifty Years. pp 158-164 (arXiv: 1711.01910), doi:10.1017/S1743921317009188

Keane E., et al., 2015, Advancing Astrophysics with the Square Kilometre Array (AASKA14), p. 40

Kondratiev V. I., et al., 2016, A\&A, 585, A128

Krause M., et al., 2018, A\&A, 611, A72

Lazarus P., Karuppusamy R., Graikou E., Caballero R. N., Champion D. J., Lee K. J., Verbiest J. P. W., Kramer M., 2016, MNRAS, 458, 868

Lenc E., et al., 2016, ApJ, 830, 38

Lenc E., et al., 2017, Publ. Astron. Soc. Australia, 34, e040

Lynch R. S., et al., 2013, ApJ, 763, 81

Lyne A. G., Pritchard R. S., Graham-Smith F., 1993, MNRAS, 265, 1003

Lyne A. G., et al., 2017, ApJ, 834, 137

Malins J. B., White S. M., Taylor G. B., Stovall K., Dowell J., 2018, Radio Science, 53, 724

Manchester R. N., 1972, ApJ, 172, 43

Manchester R. N., 1974, ApJ, 188, 637

Manchester R. N., Taylor J. H., Huguenin G. R., 1975, ApJ, 196, 83

Manchester R. N., Hobbs G. B., Teoh A., Hobbs M., 2005, AJ, 129, 1993

Mao S. A., Gaensler B. M., Haverkorn M., Zweibel E. G., Madsen G. J., McClure-Griffiths N. M., Shukurov A., Kronberg P. P., 2010, ApJ, 714, 1170

Mao S. A., et al., 2012, ApJ, 755, 21

Michilli D., et al., 2018a, MNRAS, 476, 2704

Michilli D., et al., 2018b, MNRAS, 480, 3457

Michilli D., et al., 2018c, Nature, 553, 182

Mitra D., Wielebinski R., Kramer M., Jessner A., 2003, A\&A, 398, 993

Neld A., et al., 2018, A\&A, 617, A136

$\mathrm{Ng} \mathrm{C}$., et al., 2014, MNRAS, 439, 1865

Nota T., Katgert P., 2010, A\&A, 513, A65

Noutsos A., Johnston S., Kramer M., Karastergiou A., 2008, MNRAS, 386, 1881

Noutsos A., Karastergiou A., Kramer M., Johnston S., Stappers B. W., 2009, MNRAS, 396, 1559

Noutsos A., et al., 2015, A\&A, 576, A62

Oppermann N., et al., 2012, A\&A, 542, A93

Oppermann N., et al., 2015, A\&A, 575, A118

Paragi Z., et al., 2015, Advancing Astrophysics with the Square Kilometre Array (AASKA14), p. 143

Pilia M., et al., 2016, A\&A, 586, A92

Planck Collaboration et al., 2016, A\&A, 596, A105

Polzin E. J., et al., 2018, MNRAS, 476, 1968

Radhakrishnan V., Cooke D. J., 1969, Astrophys. Lett., 3, 225

Rand R. J., Kulkarni S. R., 1989, ApJ, 343, 760

Rand R. J., Lyne A. G., 1994, MNRAS, 268, 497

Riseley C. J., et al., 2018, Publ. Astron. Soc. Australia, 35, e043

Robishaw T., et al., 2015, Advancing Astrophysics with the Square Kilometre Array (AASKA14), p. 110

Savage B. D., Wakker B. P., 2009, ApJ, 702, 1472

Schnitzeler D. H. F. M., 2012, MNRAS, 427, 664

Schnitzeler D. H. F. M., Lee K. J., 2015, MNRAS, 447, L26

Schnitzeler D. H. F. M., Lee K. J., 2017, MNRAS, 466, 378

Schnitzeler D. H. F. M., Katgert P., de Bruyn A. G., 2007, A\&A, 471, L21

Schnitzeler D. H. F. M., Katgert P., de Bruyn A. G., 2009, A\&A, 494, 611 
Sobey C., 2015, PhD thesis, University of Bonn

Sobey C., et al., 2015, MNRAS, 451, 2493

Sotomayor-Beltran C., et al., 2013, A\&A, 552, A58

Stappers B. W., et al., 2011, A\&A, 530, A80

Stovall K., et al., 2014, ApJ, 791, 67

Stovall K., et al., 2015, The Astrophysical Journal, 808, 156

Sun X.-H., Reich W., 2010, Research in Astronomy and Astrophysics, 10, 1287

Sun X. H., Reich W., 2012, A\&A, 543, A127

Sun X. H., Reich W., Waelkens A., Enßlin T. A., 2008, A\&A, 477, 573

Sun X. H., et al., 2015, ApJ, 811, 40

Tan C. M., et al., 2018, ApJ, 866, 54

Taylor J. H., Cordes J. M., 1993, ApJ, 411, 674

Taylor J. H., Manchester R. N., Lyne A. G., 1993, ApJS, 88, 529

Taylor G. B., et al., 2012, Journal of Astronomical Instrumentation, 1, 50004

Terral P., Ferrière K., 2017, A\&A, 600, A29

Thébault E., et al., 2015, Earth, Planets and Space, 67, 79

Tiburzi C., Verbiest J. P. W., 2018, in Weltevrede P., Perera B. B. P., Preston L. L., Sanidas S., eds, IAU Symposium Vol. 337, Pulsar Astrophysics the Next Fifty Years. pp 279-282 (arXiv: 1804.04040), doi: $10.1017 /$ S1743921317009760

Tingay S. J., et al., 2013, Publ. Astron. Soc. Australia, 30, 7

Van Eck C. L., et al., 2011, ApJ, 728, 97

Van Eck C. L., et al., 2017, A\&A, 597, A98

Van Eck C. L., et al., 2018, A\&A, 613, A58

Wang C., Han J. L., Lai D., 2011, MNRAS, 417, 1183

Weisberg J. M., Cordes J. M., Kuan B., Devine K. E., Green J. T., Backer D. C., 2004, ApJS, 150, 317

Xue M., et al., 2017, Publ. Astron. Soc. Australia, 34, e070

Yao J. M., Manchester R. N., Wang N., 2017, ApJ, 835, 29

van Haarlem M. P., et al., 2013, A\&A, 556, A2

van Leeuwen J., Stappers B. W., 2010, A\&A, 509, A7

van Straten W., Bailes M., 2011, Publ. Astron. Soc. Australia, 28, 1 


\section{APPENDIX A: SUMMARY OF OBSERVATIONS AND RESULTS}

Table A1 summarises the pulsar observations in the 'slow' (nonrecycled) pulsar and MSP LOFAR HBA censuses (Bilous et al. 2016; Kondratiev et al. 2016, respectively). Columns 1-4 show published information for the pulsars: the pulsar name (based on B1950 or J2000 coordinates); and the RM published in the latest version of the pulsar catalogue (v 1.59), with uncertainty ( \pm ), and literature reference (all noted by an asterisk if no literature measurements are published in the pulsar catalogue). The pulsars are listed in order of RA (as used in the pulsar catalogue), with the pulsars in the 'slow' pulsar census listed first and the pulsars in the MSP census listed after. Columns 5-9 provide the details of the LOFAR observations: modified Julian date of the LOFAR observation (MJD); signal-to-noise $(\mathrm{S} / \mathrm{N})$ of the total intensity (Stokes I) pulsar profile; number of bins used to extract the Stokes parameters from the pulse profile, and the total number of pulse profile phase bins, separated by ' $\%$; the LOFAR DM and uncertainty (Bilous et al. 2016; Kondratiev et al. 2016). Columns 10-18 show the results from this work: the total observed $\mathrm{RM}\left(\mathrm{RM}_{\mathrm{obs}}\right)$, plus uncertainty; the ionospheric RM ( $\left.\mathrm{RM}_{\mathrm{ion}}\right)$, plus uncertainty; RM due to the ISM alone $\left(\mathrm{RM}_{\mathrm{ISM}}=\mathrm{RM}_{\mathrm{obs}}-\mathrm{RM}_{\mathrm{ion}}\right)$, plus uncertainty; resulting electron density-weighted average magnetic field parallel to the LoS $\left(\left\langle B_{\|}\right\rangle\right)$, plus uncertainty; and RM dispersion output from RM CLEAN $\left(\sigma_{\mathrm{RM}}\right)$.

The references in Table A1 are listed in full in alphabetical order in Table A2, along with the number of pulsars in common with this work (MSPs are identified separately), the lowest centre observing frequency, and whether the RMs were corrected for $\mathrm{RM}_{\mathrm{ion}}$. 
Table A1: Summary of RM measurements. See text for further description of columns.

\begin{tabular}{|c|c|c|c|c|c|c|c|c|c|c|c|c|c|c|c|c|c|}
\hline PSR & 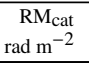 & $\begin{array}{r} \pm \\
\mathrm{rad} \mathrm{m}^{-2}\end{array}$ & ref & MJD & $\mathrm{S} / \mathrm{N}$ & Bins & $\begin{array}{r}\mathrm{DM} \\
\mathrm{pc} \mathrm{cm}^{-3} \\
\end{array}$ & $\begin{array}{r} \pm \\
\mathrm{pc} \mathrm{cm}^{-3}\end{array}$ & $\begin{array}{r}\mathrm{RM}_{\mathrm{obs}} \\
\mathrm{rad} \mathrm{m}^{-2}\end{array}$ & $\begin{array}{r} \pm \\
\mathrm{rad} \mathrm{m}^{-2}\end{array}$ & $\begin{array}{r}\mathrm{RM}_{\mathrm{ion}} \\
\mathrm{rad} \mathrm{m}^{-2}\end{array}$ & $\begin{array}{r} \pm \\
\mathrm{rad} \mathrm{m}^{-2} \\
\end{array}$ & $\begin{array}{l}\mathrm{RM}_{\text {ISM }} \\
\operatorname{rad~m}^{-2}\end{array}$ & $\begin{array}{r} \pm \\
\mathrm{rad} \mathrm{m}^{-2}\end{array}$ & $\begin{array}{r}\left\langle B_{\|}\right\rangle \\
\mu \mathrm{G} \\
\end{array}$ & $\begin{array}{r} \pm \\
\mu \mathrm{G} \\
\end{array}$ & $\begin{array}{r}\sigma_{\mathrm{RM}} \\
\mathrm{rad} \mathrm{m}^{-2} \\
\end{array}$ \\
\hline 'Slow' census & $=117$ & & & & & & & & & & & & & & & & \\
\hline B0011+47 & -15.30 & 0.70 & hmvd18 & 56773.46 & 36 & $11 / 1024$ & 30.4050 & 0.0130 & -13.06 & 0.03 & 2.50 & 0.09 & -15.56 & 0.10 & -0.631 & 0.005 & $<0.0005$ \\
\hline B $0037+56$ & 9.00 & 13.00 & r194 & 56753.42 & 38 & $43 / 1024$ & 92.5146 & 0.0025 & 18.96 & 0.08 & 3.65 & 0.16 & 15.31 & 0.18 & 0.204 & 0.003 & $<0.0003$ \\
\hline B $0045+33$ & 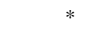 & * & * & 56703.61 & 98 & $6 / 1024$ & 39.9220 & 0.0150 & -80.14 & 0.02 & 3.14 & 0.09 & -83.27 & 0.10 & -2.570 & 0.004 & $<0.0005$ \\
\hline B $0052+51$ & -66.60 & 1.50 & hmvd18 & 56776.32 & 35 & $7 / 1024$ & 44.0127 & 0.0024 & -61.69 & 0.03 & 2.40 & 0.06 & -64.09 & 0.07 & -1.794 & 0.002 & $<0.2227$ \\
\hline B $0053+47$ & -23.00 & 22.00 & mwkj03 & 56703.59 & 19 & $51 / 1024$ & 18.1354 & 0.0013 & -31.38 & 0.10 & 2.78 & 0.09 & -34.16 & 0.13 & -2.321 & 0.011 & $<0.0004$ \\
\hline B0105+65 & -29.00 & 3.00 & $\operatorname{man} 74$ & 56784.4 & 88 & $5 / 1024$ & 30.5482 & 0.0014 & -24.37 & 0.01 & 2.71 & 0.11 & -27.09 & 0.11 & -1.092 & 0.005 & $<0.0241$ \\
\hline B0105+68 & -46.00 & 19.00 & mwkj03 & 56703.62 & 29 & $5 / 1024$ & 61.0617 & 0.0036 & -30.52 & 0.02 & 2.51 & 0.08 & -33.04 & 0.08 & -0.667 & 0.002 & $<0.1386$ \\
\hline $\mathrm{J} 0137+1654$ & . & * & * & 56703.64 & 18 & 17/1024 & 26.0838 & 0.0024 & -13.39 & 0.03 & 3.44 & 0.09 & -16.83 & 0.10 & -0.795 & 0.006 & $<0.0003$ \\
\hline B0136+57 & -94.13 & 0.08 & nsk +15 & 56753.45 & 394 & $5 / 256$ & 73.8114 & 0.0008 & -90.26 & 0.01 & 3.71 & 0.19 & -93.97 & 0.19 & -1.568 & 0.004 & $<0.2819$ \\
\hline J0152+0948 & 20.00 & 12.00 & hmvd18 & 56784.43 & 12 & $13 / 1024$ & 22.8810 & 0.0120 & 5.52 & 0.11 & 4.16 & 0.15 & 1.37 & 0.19 & 0.074 & 0.012 & $<0.0005$ \\
\hline B $0153+39$ & -46.50 & 5.50 & hmvd18 & 56773.5 & 20 & 9/1024 & 59.8330 & 0.0110 & -65.86 & 0.02 & 2.72 & 0.08 & -68.58 & 0.09 & -1.412 & 0.002 & $<0.1039$ \\
\hline $\mathrm{J} 0212+5222$ & * & * & * & 56773.52 & 23 & $3 / 1024$ & 38.2356 & 0.0005 & -11.15 & 0.02 & 2.53 & 0.08 & -13.68 & 0.08 & -0.441 & 0.003 & $<0.1114$ \\
\hline В0226+70 & -56.00 & 21.00 & mwkj03 & 56703.68 & 21 & $5 / 1024$ & 46.6794 & 0.0016 & -41.56 & 0.02 & 2.25 & 0.06 & -43.81 & 0.06 & -1.156 & 0.002 & $<0.0003$ \\
\hline B $0301+19$ & -8.30 & 0.30 & $\operatorname{man} 74$ & 56703.69 & 146 & $2 / 1024$ & 15.6568 & 0.0004 & -5.48 & 0.02 & 2.95 & 0.06 & -8.43 & 0.06 & -0.663 & 0.006 & $<0.1734$ \\
\hline B $0320+39$ & 56.30 & 1.00 & fdr15 & 56703.72 & 625 & $1 / 1024$ & 26.1898 & 0.0009 & 62.32 & 0.02 & 2.33 & 0.04 & 59.99 & 0.05 & 2.822 & 0.003 & $<0.3189$ \\
\hline B0331+45 & -41.00 & 20.00 & rl94 & 56747.65 & 143 & $3 / 512$ & 47.1457 & 0.0003 & 10.57 & 0.05 & 4.98 & 0.08 & 5.60 & 0.09 & 0.146 & 0.003 & 0.0000 \\
\hline B0402+61 & 9.00 & 3.00 & hl87 & 56747.67 & 48 & $2 / 1024$ & 65.4053 & 0.0034 & 12.56 & 0.02 & 4.38 & 0.08 & 8.18 & 0.08 & 0.154 & 0.002 & $<0.3028$ \\
\hline B $0410+69$ & -31.60 & 4.80 & hmvd18 & 56773.55 & 77 & $3 / 1024$ & 27.4460 & 0.0004 & -19.09 & 0.02 & 2.33 & 0.06 & -21.42 & 0.06 & -0.962 & 0.004 & 0.0000 \\
\hline $\mathrm{J} 0417+35$ & $*$ & * & & 56773.56 & 31 & $4 / 1024$ & 48.5336 & 0.0012 & 45.04 & 0.06 & 3.03 & 0.07 & 42.01 & 0.10 & 1.066 & 0.003 & $<0.0004$ \\
\hline $\mathrm{J} 0435+2749$ & 2.00 & 0.00 & bfrs 18 & 56773.58 & 20 & $9 / 512$ & 53.1819 & 0.0002 & 4.21 & 0.07 & 3.25 & 0.06 & 0.96 & 0.09 & 0.022 & 0.003 & $<0.0005$ \\
\hline B $0450+55$ & 10.00 & 3.00 & hl87 & 56772.61 & 275 & $1 / 1024$ & 14.5900 & 0.0002 & 8.74 & 0.01 & 2.94 & 0.11 & 5.79 & 0.11 & 0.489 & 0.011 & $<0.0004$ \\
\hline B $0523+11$ & 37.00 & 2.00 & jhv+05 & 56703.77 & 32 & $28 / 512$ & 79.4180 & 0.0130 & 22.18 & 0.13 & 2.07 & 0.10 & 20.11 & 0.17 & 0.312 & 0.003 & 0.0000 \\
\hline B $0525+21$ & -39.60 & 0.20 & $\operatorname{man} 72$ & 56747.71 & 746 & $1 / 1024$ & 50.8695 & 0.0013 & -34.55 & 0.01 & 5.47 & 0.06 & -40.02 & 0.07 & -0.969 & 0.002 & $<0.2558$ \\
\hline B0531+21 & -42.30 & 0.50 & $\operatorname{man} 72$ & 56703.78 & 120 & $1 / 64$ & 56.7712 & 0.0002 & -43.70 & 0.01 & 1.74 & 0.08 & -45.44 & 0.08 & -0.986 & 0.002 & $<0.2542$ \\
\hline B $0540+23$ & 2.70 & 0.60 & $\mathrm{jkk}+07$ & 56779.57 & 39 & $1 / 256$ & 77.7026 & 0.0010 & 5.95 & 0.01 & 3.10 & 0.10 & 2.85 & 0.10 & 0.045 & 0.002 & $<0.1797$ \\
\hline J $0611+30$ & * & * & * & 56772.64 & 78 & $2 / 1024$ & 45.2551 & 0.0016 & 17.91 & 0.02 & 3.45 & 0.08 & 14.46 & 0.08 & 0.394 & 0.003 & 0.0000 \\
\hline B $0609+37$ & 23.00 & 9.00 & wck+04 & 56772.66 & 50 & $1 / 512$ & 27.1550 & 0.0003 & 37.73 & 0.02 & 3.22 & 0.05 & 34.51 & 0.06 & 1.566 & 0.003 & $<0.0005$ \\
\hline B $0626+24$ & 69.50 & 0.20 & wck +04 & 56747.76 & 160 & $2 / 512$ & 84.1762 & 0.0050 & 80.41 & 0.02 & 4.70 & 0.09 & 75.70 & 0.09 & 1.108 & 0.002 & $<0.2716$ \\
\hline B $0643+80$ & -32.00 & 3.00 & hl187 & 56747.78 & 97 & $1 / 1024$ & 33.3188 & 0.0007 & -28.87 & 0.01 & 2.89 & 0.06 & -31.77 & 0.07 & -1.175 & 0.003 & $<0.2068$ \\
\hline B0656+14 & 23.00 & 0.30 & $\mathrm{jkk}+07$ & 56747.75 & 15 & $14 / 1024$ & 14.0762 & 0.0024 & 28.00 & 0.02 & 5.26 & 0.07 & 22.73 & 0.08 & 1.990 & 0.008 & $<0.0004$ \\
\hline B $0655+64$ & -7.00 & 6.00 & hl87 & 56772.68 & 112 & $3 / 1024$ & 8.7739 & 0.0003 & -15.36 & 0.02 & 2.71 & 0.06 & -18.07 & 0.06 & -2.537 & 0.011 & $<0.0003$ \\
\hline B $0751+32$ & -7.00 & 5.00 & hl87 & 56772.72 & 104 & $11 / 1024$ & 39.9863 & 0.0014 & 7.60 & 0.03 & 3.42 & 0.08 & 4.18 & 0.09 & 0.129 & 0.003 & $<0.1472$ \\
\hline B $0809+74$ & -14.00 & 0.07 & nsk +15 & 56747.8 & 661 & $2 / 1024$ & 5.7507 & 0.0005 & -11.07 & 0.01 & 2.84 & 0.06 & -13.91 & 0.06 & -2.980 & 0.016 & $<0.1743$ \\
\hline B $0823+26$ & 5.38 & 0.06 & nsk +15 & 56747.82 & 1589 & $2 / 1024$ & 19.4763 & 0.0002 & 8.93 & 0.01 & 3.87 & 0.10 & 5.05 & 0.10 & 0.320 & 0.008 & $<0.0953$ \\
\hline B $0841+80$ & -23.20 & 3.10 & hmvd18 & 56747.83 & 23 & $7 / 1024$ & 34.8121 & 0.0031 & -19.42 & 0.03 & 2.36 & 0.04 & -21.78 & 0.05 & -0.771 & 0.002 & $<0.0004$ \\
\hline B $0917+63$ & -18.90 & 2.30 & hmvd18 & 56747.89 & 164 & $2 / 1024$ & 13.1542 & 0.0002 & -12.81 & 0.01 & 2.12 & 0.06 & -14.93 & 0.06 & -1.399 & 0.007 & $<0.1745$ \\
\hline B $0940+16$ & 53.00 & 12.00 & h187 & 56687.1 & 21 & $2 / 1024$ & 20.3402 & 0.0018 & 18.23 & 0.02 & 0.86 & 0.06 & 17.36 & 0.07 & 1.052 & 0.005 & 0.0000 \\
\hline $\mathrm{J} 0943+2253$ & $*$ & * & * & 56747.86 & 33 & $3 / 1024$ & 27.2676 & 0.0016 & 19.76 & 0.02 & 3.33 & 0.08 & 16.43 & 0.08 & 0.742 & 0.004 & $<0.0004$ \\
\hline B $0943+10$ & 13.30 & 0.50 & $\mathrm{tm} 193$ & 56779.82 & 882 & $1 / 1024$ & 15.3185 & 0.0009 & 17.23 & 0.02 & 3.13 & 0.08 & 14.10 & 0.08 & 1.134 & 0.008 & $<0.1470$ \\
\hline $\mathrm{J} 0947+2740$ & 32.00 & 0.00 & bfrs 18 & 56747.88 & 24 & $5 / 1024$ & 28.8860 & 0.0260 & 26.28 & 0.02 & 3.03 & 0.06 & 23.25 & 0.06 & 0.992 & 0.003 & $<0.1104$ \\
\hline B $1112+50$ & -0.10 & 0.80 & fdr15 & 56747.93 & 500 & $1 / 1024$ & 9.1863 & 0.0003 & 4.44 & 0.01 & 2.02 & 0.07 & 2.41 & 0.07 & 0.324 & 0.012 & $<0.1194$ \\
\hline B1133+16 & 3.97 & 0.07 & nsk +15 & 56687.11 & 2220 & $1 / 1024$ & 4.8407 & 0.0003 & 5.00 & 0.01 & 0.89 & 0.06 & 4.11 & 0.06 & 1.046 & 0.019 & $<0.0005$ \\
\hline $\mathrm{J} 1238+2152$ & * & * & * & 56687.14 & 133 & $2 / 1024$ & 17.9706 & 0.0031 & 5.21 & 0.01 & 0.80 & 0.05 & 4.41 & 0.06 & 0.303 & 0.005 & $<0.1848$ \\
\hline $\mathrm{J} 1246+2253$ & * & * & * & 56747.99 & 21 & 29/1024 & 17.7978 & 0.0018 & 6.01 & 0.03 & 2.42 & 0.09 & 3.59 & 0.09 & 0.248 & 0.008 & $<0.0004$ \\
\hline $\mathrm{J} 1313+0931$ & * & * & * & 56779.92 & 152 & $1 / 1024$ & 12.0406 & 0.0000 & 4.49 & 0.02 & 2.17 & 0.10 & 2.32 & 0.11 & 0.237 & 0.013 & $<0.1704$ \\
\hline B1322+83 & -23.20 & 1.10 & fdr15 & 56687.19 & 90 & $1 / 1024$ & 13.3162 & 0.0008 & -23.32 & 0.02 & 0.35 & 0.05 & -23.67 & 0.05 & -2.190 & 0.005 & $<0.0005$ \\
\hline B1508+55 & 1.28 & 0.06 & nsk +15 & 56687.26 & 3384 & $1 / 1024$ & 19.6189 & 0.0013 & 2.04 & 0.01 & 0.55 & 0.06 & 1.49 & 0.06 & 0.094 & 0.005 & $<0.1733$ \\
\hline B1530+27 & 1.00 & 0.30 & wck+04 & 56703.26 & 72 & $7 / 1024$ & 14.6910 & 0.0160 & 4.26 & 0.02 & 0.89 & 0.08 & 3.37 & 0.08 & 0.283 & 0.008 & $<0.1293$ \\
\hline B1541+09 & 21.00 & 2.00 & hl87 & 56780.02 & 544 & $1 / 102$ & 34.9758 & 0.0016 & 17.61 & 0.01 & 1.66 & 09 & 15.95 & 0.09 & 0.562 & 0.004 & $<0.1028$ \\
\hline $\mathrm{J} 1612+2008$ & 22.00 & 3.00 & $\mathrm{blr}+13$ & 56687.25 & 15 & 9/1024 & 19.5082 & 0.0032 & 23.01 & 0.02 & 0.75 & 0.06 & 22.26 & 0.07 & 1.406 & 0.005 & 0.0000 \\
\hline $\mathrm{J} 1627+1419$ & 18.30 & 5.90 & hmvd18 & 56687.29 & 64 & $1 / 1024$ & 32.1670 & 0.0008 & 19.85 & 0.02 & 1.24 & 0.06 & 18.61 & 0.06 & 0.713 & 0.003 & $<0.0009$ \\
\hline B $1633+24$ & 31.00 & 4.00 & wck+04 & 56748.14 & 135 & $2 / 1024$ & 24.2671 & 0.0044 & 23.81 & 0.03 & 1.85 & 0.06 & 21.97 & 0.07 & 1.115 & 0.004 & $<0.0003$ \\
\hline $\mathrm{J} 1645+1012$ & & * & & 56687.33 & 87 & $1 / 1024$ & 36.1713 & 0.0002 & 32.25 & 0.02 & 2.05 & 0.10 & 30.20 & 0.10 & 1.029 & 0.004 & 0.0000 \\
\hline $\mathrm{J} 1649+2533$ & 29.70 & 1.00 & hmvd18 & 56748.12 & 25 & $11 / 1024$ & 34.4622 & 0.0078 & 31.39 & 0.03 & 1.87 & 0.05 & 29.52 & 0.06 & 1.055 & 0.003 & $<0.0987$ \\
\hline $\mathrm{J} 1652+2651$ & 34.50 & 0.70 & hmvd18 & 56748.11 & 33 & $21 / 1024$ & 40.8024 & 0.0002 & 35.56 & 0.07 & 1.85 & 0.04 & 33.71 & 0.08 & 1.018 & 0.003 & $<0.1472$ \\
\hline
\end{tabular}


Table A1 - continued from previous page

\begin{tabular}{|c|c|c|c|c|c|c|c|c|c|c|c|c|c|c|c|c|c|}
\hline PSR & $\begin{array}{r}\mathrm{RM}_{\mathrm{cat}} \\
\mathrm{rad} \mathrm{m}^{-2}\end{array}$ & $\begin{array}{r} \pm \\
\mathrm{rad} \mathrm{m}^{-2}\end{array}$ & ref & MJD & $\mathrm{S} / \mathrm{N}$ & Bins & $\begin{array}{r}\mathrm{DM} \\
\mathrm{pc} \mathrm{cc^{-3 }}\end{array}$ & $\begin{array}{r} \pm \\
\mathrm{pc} \mathrm{cm}^{-3}\end{array}$ & $\begin{array}{r}\mathrm{RM}_{\mathrm{obs}} \\
\mathrm{rad} \mathrm{m}^{-2}\end{array}$ & $\begin{array}{r} \pm \\
\mathrm{rad} \mathrm{m}^{-2}\end{array}$ & $\begin{array}{r}\mathrm{RM}_{\mathrm{ion}} \\
\mathrm{rad} \mathrm{m}^{-2}\end{array}$ & $\begin{array}{r} \pm \\
\mathrm{rad} \mathrm{m}^{-2}\end{array}$ & $\begin{array}{r}\mathrm{RM}_{\mathrm{ISM}} \\
\mathrm{rad} \mathrm{m}^{-2}\end{array}$ & $\begin{array}{r} \pm \\
\mathrm{rad} \mathrm{m}^{-2}\end{array}$ & $\begin{array}{c}\left\langle B_{\|}\right\rangle \\
\mu \mathrm{G}\end{array}$ & $\begin{array}{r} \pm \\
\mu \mathrm{G}\end{array}$ & $\begin{array}{r}\sigma_{\mathrm{RM}} \\
\mathrm{rad} \mathrm{m}^{-2}\end{array}$ \\
\hline $\mathrm{J} 1720+2150$ & 50.00 & 3.70 & hmvd18 & 56748.15 & 16 & $5 / 1024$ & 40.7190 & 0.0350 & 54.76 & 0.03 & 1.82 & 0.06 & 52.94 & 0.07 & 1.602 & 0.003 & $<0.1456$ \\
\hline B1737+13 & 64.40 & 1.60 & wck +04 & 56687.3 & 161 & $2 / 1024$ & 48.6682 & 0.0004 & 68.22 & 0.03 & 1.58 & 0.07 & 66.65 & 0.08 & 1.687 & 0.002 & $<0.0005$ \\
\hline $\mathrm{J} 1741+2758$ & 49.90 & 5.70 & hmvd18 & 56773.14 & 93 & $5 / 1024$ & 29.1449 & 0.0001 & 56.03 & 0.03 & 1.34 & 0.06 & 54.70 & 0.07 & 2.312 & 0.004 & 0.0000 \\
\hline $\mathrm{J} 1746+2245$ & 68.70 & 3.70 & hmvd18 & 56784.14 & 25 & $3 / 1024$ & 49.8543 & 0.0057 & 73.84 & 0.08 & 1.57 & 0.10 & 72.28 & 0.12 & 1.786 & 0.004 & $<0.0003$ \\
\hline $\mathrm{J} 1746+2540$ & 93.20 & 1.50 & hmvd18 & 56784.17 & 13 & $13 / 1024$ & 51.2044 & 0.0033 & 96.46 & 0.08 & 1.71 & 0.10 & 94.75 & 0.12 & 2.280 & 0.004 & $<0.0003$ \\
\hline $\mathrm{J} 1752+2359$ & * & * & & 56783.18 & 20 & $9 / 1024$ & 36.1964 & 0.0006 & 88.64 & 0.06 & 1.59 & 0.09 & 87.05 & 0.11 & 2.963 & 0.005 & $<0.0005$ \\
\hline B1753+52 & 26.60 & 1.10 & hmvd18 & 56785.18 & 17 & $5 / 1024$ & 35.0096 & 0.0064 & 29.25 & 0.08 & 1.31 & 0.07 & 27.94 & 0.11 & 0.983 & 0.005 & $<0.0672$ \\
\hline $\mathrm{J} 1758+3030$ & 70.40 & 2.20 & hmvd18 & 56784.19 & 157 & $4 / 1024$ & 35.0674 & 0.0014 & 81.39 & 0.07 & 1.85 & 0.10 & 79.54 & 0.12 & 2.794 & 0.005 & $<0.0005$ \\
\hline B1811+40 & 47.00 & 6.00 & hl87 & 56780.05 & 103 & $2 / 1024$ & 41.5566 & 0.0002 & 50.53 & 0.02 & 1.15 & 0.06 & 49.38 & 0.06 & 1.464 & 0.002 & $<0.1568$ \\
\hline $\mathrm{J} 1821+1715$ & 107.50 & 1.20 & hmvd18 & 56703.3 & 25 & $7 / 1024$ & 60.2844 & 0.0025 & 111.21 & 0.08 & 1.85 & 0.10 & 109.36 & 0.13 & 2.235 & 0.003 & $<0.0665$ \\
\hline $\mathrm{J} 1838+1650$ & 66.50 & 2.70 & hmvd18 & 56801.09 & 84 & $1 / 1024$ & 32.9516 & 0.0010 & 74.36 & 0.02 & 1.71 & 0.12 & 72.65 & 0.12 & 2.716 & 0.005 & $<0.0772$ \\
\hline B1839+56 & -5.00 & 0.70 & fdr15 & 56789.09 & 532 & $1 / 1024$ & 26.7716 & 0.0002 & -2.58 & 0.01 & 1.27 & 0.06 & -3.85 & 0.06 & -0.177 & 0.004 & $<0.0917$ \\
\hline B1839+09 & 53.00 & 5.00 & hl87 & 56783.19 & 110 & $1 / 1024$ & 49.1579 & 0.0043 & 53.23 & 0.02 & 2.04 & 0.10 & 51.19 & 0.10 & 1.283 & 0.003 & $<0.1000$ \\
\hline B1842+14 & 109.00 & 1.30 & jhv +05 & 56703.31 & 145 & $3 / 1024$ & 41.4856 & 0.0006 & 120.84 & 0.04 & 2.19 & 0.11 & 118.65 & 0.12 & 3.524 & 0.004 & $<0.1303$ \\
\hline $\mathrm{J} 1849+2423$ & 12.80 & 1.20 & hmvd18 & 56788.06 & 9 & $4 / 512$ & 62.2677 & 0.0016 & 16.82 & 0.03 & 1.64 & 0.05 & 15.18 & 0.06 & 0.300 & 0.001 & $<0.0005$ \\
\hline B1848+13 & 152.70 & 0.60 & jkk+07 & 56703.34 & 10 & $11 / 512$ & 60.1396 & 0.0066 & 157.58 & 0.10 & 2.65 & 0.12 & 154.93 & 0.16 & 3.174 & 0.004 & $<0.0005$ \\
\hline B1848+12 & 158.00 & 16.00 & rl94 & 56687.43 & 53 & $3 / 1024$ & 70.6333 & 0.0017 & 152.53 & 0.03 & 2.73 & 0.12 & 149.80 & 0.12 & 2.613 & 0.003 & $<0.2659$ \\
\hline B1905+39 & 5.20 & 0.30 & fdr15 & 56789.13 & 119 & $1 / 1024$ & 30.9660 & 0.0140 & 6.88 & 0.01 & 1.47 & 0.08 & 5.41 & 0.09 & 0.215 & 0.004 & $<0.1029$ \\
\hline $\mathrm{J} 1912+2525$ & 12.30 & 7.00 & hmvd18 & 56788.23 & 42 & $2 / 1024$ & 37.8474 & 0.0016 & 34.69 & 0.02 & 2.04 & 0.09 & 32.65 & 0.10 & 1.063 & 0.004 & $<0.0621$ \\
\hline B $1918+26$ & 20.30 & 1.30 & hmvd18 & 56748.21 & 95 & $2 / 1024$ & 27.7088 & 0.0008 & 28.11 & 0.02 & 2.07 & 0.07 & 26.04 & 0.07 & 1.158 & 0.004 & $<0.0005$ \\
\hline B1919+21 & -16.99 & 0.05 & nsk +15 & 56788.21 & 2054 & $1 / 1024$ & 12.4440 & 0.0006 & -15.04 & 0.02 & 2.01 & 0.10 & -17.05 & 0.10 & -1.688 & 0.012 & $<0.0005$ \\
\hline B 1929+10 & -6.87 & 0.02 & jhv +05 & 56781.21 & 314 & $1 / 1024$ & 3.1832 & 0.0002 & -5.27 & 0.01 & 1.86 & 0.14 & -7.14 & 0.14 & -2.762 & 0.065 & $<0.0863$ \\
\hline B1944+17 & -28.00 & 0.40 & hl87 & 56801.14 & 29 & 9/1024 & 16.1356 & 0.0073 & -43.64 & 0.02 & 1.7 & 0.10 & -45.34 & 0.11 & -3.462 & 0.010 & $<0.1774$ \\
\hline B $1946+35$ & 116.00 & 6.00 & hl87 & 56748.23 & 28 & $70 / 1024$ & 129.3675 & 0.0008 & 121.07 & 0.09 & 2.11 & 0.06 & 118.96 & 0.11 & 1.133 & 0.001 & $<0.3047$ \\
\hline B1953+50 & -23.84 & 0.05 & nsk +15 & 56789.06 & 155 & $1 / 1024$ & 31.9827 & 0.0001 & -22.50 & 0.01 & 1.31 & 0.06 & -23.82 & 0.06 & -0.918 & 0.003 & $<0.1041$ \\
\hline $\mathrm{J} 1956+0838$ & * & * & $*$ & 56781.27 & 6 & $25 / 1024$ & 67.0870 & 0.0240 & -111.05 & 0.11 & 2.44 & 0.12 & -113.49 & 0.16 & -2.084 & 0.004 & 0.0000 \\
\hline J2002+1637 & * & * & * & 56773.22 & 4 & $8 / 256$ & 94.5810 & 0.0480 & -39.29 & 0.09 & 1.98 & 0.10 & -41.27 & 0.13 & -0.538 & 0.002 & $<0.0004$ \\
\hline $\mathrm{J} 2007+0809$ & * & * & * & 56779.29 & 10 & $47 / 512$ & 53.3940 & 0.0370 & -130.49 & 0.07 & 2.84 & 0.13 & -133.32 & 0.15 & -3.076 & 0.005 & $<0.0005$ \\
\hline $\mathrm{J} 2007+0910$ & -73.00 & 15.00 & hmvd18 & 56784.25 & 36 & $5 / 1024$ & 48.7293 & 0.0007 & -74.57 & 0.09 & 3.24 & 0.09 & -77.81 & 0.13 & -1.967 & 0.004 & 0.0000 \\
\hline $\mathrm{J} 2017+2043$ & -168.00 & 3.10 & hmvd18 & 56772.32 & 32 & $8 / 1024$ & 60.4906 & 0.0099 & -160.93 & 0.03 & 2.68 & 0.09 & -163.61 & 0.10 & -3.332 & 0.002 & $<0.0004$ \\
\hline B2016+28 & -34.60 & 1.40 & $\operatorname{man} 72$ & 56781.23 & 1255 & $1 / 1024$ & 14.1839 & 0.0013 & -33.14 & 0.01 & 1.76 & 0.11 & -34.90 & 0.11 & -3.032 & 0.012 & $<0.1741$ \\
\hline B2020+28 & -74.70 & 0.30 & $\operatorname{man} 74$ & 56748.24 & 252 & $1 / 1024$ & 24.6311 & 0.0002 & -72.56 & 0.02 & 2.48 & 0.06 & -75.04 & 0.06 & -3.753 & 0.004 & $<0.1595$ \\
\hline B2021+51 & -6.50 & 0.90 & $\operatorname{man} 72$ & 56789.31 & 126 & $1 / 1024$ & 22.5497 & 0.0006 & -4.31 & 0.01 & 2.42 & 0.08 & -6.73 & 0.08 & -0.368 & 0.005 & $<0.1258$ \\
\hline B2022+50 & 42.60 & 1.20 & hmvd18 & 56789.08 & 75 & $1 / 1024$ & 32.9882 & 0.0004 & 46.11 & 0.02 & 1.28 & 0.06 & 44.84 & 0.06 & 1.675 & 0.003 & $<0.0005$ \\
\hline $\mathrm{J} 2036+2835$ & * & * & $*$ & 56801.17 & 23 & $3 / 1024$ & 84.2174 & 0.0064 & -156.45 & 0.03 & 1.69 & 0.12 & -158.14 & 0.12 & -2.313 & 0.002 & $<0.0006$ \\
\hline B2034+19 & -97.00 & 10.00 & wck +04 & 56773.27 & 91 & $1 / 1024$ & 36.8916 & 0.0000 & -108.46 & 0.02 & 2.22 & 0.10 & -110.69 & 0.10 & -3.696 & 0.004 & $<0.1957$ \\
\hline B2036+53 & -102.30 & 1.90 & hmvd18 & 56789.33 & 8 & $25 / 1024$ & 160.1960 & 0.0120 & -100.56 & 0.10 & 2.49 & 0.08 & -103.05 & 0.13 & -0.793 & 0.001 & $<0.0005$ \\
\hline $\mathrm{J} 2043+2740$ & & * & & 56784.28 & 233 & $1 / 256$ & 21.0206 & 0.0002 & -93.08 & 0.01 & 3.06 & 0.12 & -96.14 & 0.12 & -5.635 & 0.009 & $<0.1766$ \\
\hline B2044+15 & -100.00 & 5.00 & wck+04 & 56773.25 & 72 & $5 / 1024$ & 39.8180 & 0.0005 & -88.02 & 0.06 & 2.18 & 0.09 & -90.20 & 0.11 & -2.791 & 0.004 & $<0.0940$ \\
\hline B2045+56 & 0.60 & 1.90 & hmvd18 & 56789.16 & 54 & $1 / 512$ & 101.7903 & 0.0001 & 2.79 & 0.02 & 1.54 & 0.08 & 1.25 & 0.08 & 0.015 & 0.001 & $<0.0005$ \\
\hline B2053+21 & -100.00 & 7.00 & $\mathrm{hr} 10$ & 56773.34 & 100 & $1 / 1024$ & 36.3496 & 0.0003 & -86.71 & 0.02 & 2.69 & 0.08 & -89.40 & 0.08 & -3.030 & 0.003 & $<0.0961$ \\
\hline $\mathrm{J} 2111+2106$ & -75.30 & 0.80 & $\mathrm{blr}+13$ & 56773.31 & 15 & $9 / 1024$ & 59.2964 & 0.0035 & -72.47 & 0.10 & 2.51 & 0.09 & -74.99 & 0.14 & -1.558 & 0.003 & 0.0000 \\
\hline B2110+27 & -37.00 & 7.00 & wck +04 & 56784.29 & 425 & $1 / 1024$ & 25.1111 & 0.0002 & -56.95 & 0.02 & 3.23 & 0.13 & -60.18 & 0.13 & -2.953 & 0.008 & 0.0000 \\
\hline B2113+14 & -25.00 & 8.00 & hl87 & 56703.48 & 21 & $45 / 1024$ & 56.2044 & 0.0061 & -35.30 & 0.07 & 3.74 & 0.09 & -39.04 & 0.11 & -0.856 & 0.003 & $<0.0003$ \\
\hline $\mathrm{J} 2139+2242$ & -86.00 & 0.40 & hmvd18 & 56753.38 & 64 & $1 / 1024$ & 44.1597 & 0.0008 & -83.13 & $0 . c$ & 4.25 & 0.22 & -87.38 & 0.22 & -2.438 & 0.008 & $<0.1608$ \\
\hline B2148+63 & -156.50 & 0.30 & fdr15 & 56786.34 & 54 & $26 / 512$ & 129.7229 & 0.0055 & -155.62 & 0.03 & 1.98 & 0.09 & -157.60 & 0.09 & -1.497 & 0.001 & $<0.2486$ \\
\hline $\mathrm{J} 2155+2813$ & -131.80 & 4.50 & hmvd18 & 56784.31 & 31 & $14 / 1024$ & 77.1309 & 0.0043 & -129.30 & 0.06 & 3.36 & 0.13 & -132.67 & 0.14 & -2.119 & 0.003 & $<0.1351$ \\
\hline B2154+40 & -32.60 & 3.00 & fdr15 & 56687.54 & 321 & $1 / 1024$ & 71.1239 & 0.0022 & -39.68 & 0.01 & 2.35 & 0.12 & -42.03 & 0.12 & -0.728 & 0.003 & $<0.2118$ \\
\hline $\mathrm{J} 2205+1444$ & -27.50 & 3.40 & hmvd18 & 56703.5 & 10 & $8 / 1024$ & 36.7460 & 0.0620 & -21.76 & 0. & 3.76 & 0.08 & -25.51 & 0.11 & -0.855 & 0.005 & $<0.0003$ \\
\hline B $2210+29$ & -168.00 & 5.00 & wck +04 & 56784 & 36 & $8 / 1024$ & 74.5 & 0.0 & -165.23 & 0. & 3.44 & 0.12 & -168.66 & 0.14 & -2.788 & 0.003 & $<0.2767$ \\
\hline $\mathrm{J} 2215+1538$ & -18.70 & 1.00 & hmvd18 & 56703.55 & 16 & $6 / 1024$ & 29.2404 & 0.0012 & -16.21 & 0.03 & 3.81 & 0.09 & -20.02 & 0.09 & -0.843 & 0.005 & $<0.0004$ \\
\hline В2217+47 & -35.93 & 0.06 & nsk +15 & 56687.56 & 3281 & $1 / 1024$ & 43.4862 & 0.0060 & -33.60 & 0.01 & 2.12 & 0.10 & -35.73 & 0.10 & -1.012 & 0.004 & $<0.0360$ \\
\hline $\mathrm{J} 2222+2923$ & * & * & * & 56773.4 & 12 & $8 / 512$ & 49.4128 & 0.0011 & -92.96 & 0.07 & 2.75 & 0.08 & -95.71 & 0.11 & -2.386 & 0.003 & $<0.0005$ \\
\hline B2224+65 & -22.99 & 0.07 & nsk +15 & 56784.36 & 232 & $1 / 1024$ & 36.4436 & 0.0005 & -20.05 & م0 & 2.69 & 0.10 & -22.73 & 0.10 & -0.769 & 0.004 & $<0.1735$ \\
\hline B2227+61 & -125.00 & 22.00 & mwkj03 & 56783. & 52 & $14 / 512$ & 124.6388 & 0.0031 & -104.22 & 0.03 & 1.69 & 0.09 & -105.91 & 0.09 & -1.047 & 0.001 & $<0.2237$ \\
\hline B2241+69 & -30.00 & 30.00 & mwkj03 & 56784.34 & 67 & $1 / 1024$ & 40.8604 & 0.0007 & -14.16 & 0.02 & 2.64 & 0.09 & -16.80 & 0.09 & -0.506 & 0.003 & $<0.0005$ \\
\hline J2253+1516 & -32.00 & 3.00 & hmvd18 & 56703.53 & 39 & $18 / 1024$ & 29.2045 & 0.0017 & -27.14 & 0.07 & 3.84 & 0.09 & -30.98 & 0.11 & -1.307 & 0.006 & $<0.0004$ \\
\hline & -75.50 & 4.00 & wck+04 & 56773.41 & 215 & $1 / 1024$ & 49.5845 & 0.0012 & -84.27 & 0.03 & 2.77 & 0.10 & -87.04 & 0.10 & -2.163 & 0.003 & $<0.0697$ \\
\hline
\end{tabular}


Table A2. Summary of the references for published RMs in Table A1.

\begin{tabular}{llll}
\hline \hline Reference & $\begin{array}{l}\text { N pulsars in common } \\
\text { 'slow' + MSP }\end{array}$ & $\begin{array}{l}\text { (lowest) Centre frequency } \\
\text { (MHz) }\end{array}$ & $\begin{array}{l}\text { Ionospheric RM corrected } \\
\text { (Yes/No) }\end{array}$ \\
\hline bfrs18 (Brinkman et al. 2018) & 2 & 327 & $\mathrm{~N}$ \\
blr+13 (Boyles et al. 2013) & 2 & 820 & $\mathrm{~N}$ \\
dhm+15 (Dai et al. 2015) & 3 MSPs & 730 & $\mathrm{Y}$ \\
fdr15 (Force et al. 2015) & 8 & 1100 & $\mathrm{~N}$ \\
h187 (Hamilton \& Lyne 1987) & 14 & 408 & $\mathrm{Y}$ \\
hmvd18 (Han et al. 2018) & $32+4$ MSPs & 774 & $\mathrm{Y}$ \\
hr10 (Hankins \& Rankin 2010) & 1 & 50 & $\mathrm{~N}$ \\
jhv+05 (Johnston et al. 2005) & 3 & 1369 & $\mathrm{Y}$ \\
jkk+07 (Johnston et al. 2007) & 3 & 690 & $\mathrm{~N}$ \\
br+13 (Lynch et al. 2013) & 1 MSP & $350 / 820$ & $\mathrm{~N}$ \\
man72 (Manchester 1972) & 4 & 250 & $\mathrm{Y}$ \\
man74 (Manchester 1974) & 3 & 250 & $\mathrm{Y}$ \\
mwkj03 (Mitra et al. 2003) & 5 & 1400 & $\mathrm{~N}$ \\
nsk+15 (Noutsos et al. 2015) & $9+3$ MSPs & 150 & $\mathrm{Y}$ \\
r194 (Rand \& Lyne 1994) & 3 & 1400 & $\mathrm{~N}$ \\
tm193 (Taylor et al. 1993) & 1 & various & various \\
wck+04 (Weisberg et al. 2004) & 10 & 430 & $\mathrm{Y}$ \\
\hline
\end{tabular}

\begin{tabular}{|c|c|c|c|c|c|c|c|c|c|c|c|c|c|c|c|c|c|}
\hline PSR & $\begin{array}{r}\mathrm{RM}_{\text {cat }} \\
\mathrm{rad} \mathrm{m}^{-2}\end{array}$ & $\begin{array}{r} \pm \\
\mathrm{rad} \mathrm{m}^{-2}\end{array}$ & ref & MJD & $\mathrm{S} / \mathrm{N}$ & Bins & $\begin{array}{r}\mathrm{DM} \\
\mathrm{pc} \mathrm{cm}^{-3}\end{array}$ & $\mathrm{pc} \mathrm{cm}^{-3}$ & $\begin{array}{r}\mathrm{RM}_{\text {obs }} \\
\mathrm{rad} \mathrm{m}^{-2}\end{array}$ & $\begin{array}{r}\quad \pm \\
\mathrm{rad} \mathrm{m}^{-2}\end{array}$ & $\begin{array}{r}\mathrm{RM}_{\text {ion }} \\
\mathrm{rad} \mathrm{m}^{-2}\end{array}$ & $\begin{array}{r} \pm \\
\mathrm{rad} \mathrm{m}^{-2}\end{array}$ & $\begin{array}{l}\mathrm{RM}_{\mathrm{ISM}} \\
\mathrm{rad} \mathrm{m}^{-2}\end{array}$ & $\begin{array}{r} \pm \\
\mathrm{rad} \mathrm{m}^{-2}\end{array}$ & $\begin{array}{r}\left\langle B_{\|}\right\rangle \\
\mu \mathrm{G}\end{array}$ & $\begin{array}{r} \pm \\
\mu \mathrm{G}\end{array}$ & $\begin{array}{r}\sigma_{\mathrm{RM}} \\
\mathrm{rad} \mathrm{m}^{-2}\end{array}$ \\
\hline B2303+46 & -25.00 & 11.00 & hmvd18 & 56773.43 & 37 & $3 / 1024$ & 62.0676 & 0.0036 & -19.64 & 0.02 & 2.44 & 0.09 & -22.08 & 0.09 & -0.438 & 0.002 & $<0.0958$ \\
\hline B $2306+55$ & -34.00 & 3.00 & hl87 & 56785.24 & 81 & $4 / 1024$ & 46.5391 & 0.0004 & -27.68 & 0.02 & 1.67 & 0.07 & -29.35 & 0.08 & -0.777 & 0.003 & $<0.1870$ \\
\hline B2310+42 & 4.40 & 0.10 & fdr15 & 56687.59 & 318 & $1 / 1024$ & 17.2769 & 0.0003 & 7.03 & 0.01 & 2.06 & 0.09 & 4.97 & 0.09 & 0.355 & 0.008 & $<0.2096$ \\
\hline B2315+21 & -37.00 & 3.00 & hl87 & 56687.52 & 257 & $1 / 1024$ & 20.8696 & 0.0003 & -35.28 & 0.01 & 2.67 & 0.13 & -37.94 & 0.13 & -2.240 & 0.009 & $<0.1231$ \\
\hline MSP census & $=19$ & & & & & & & & & & & & & & & & \\
\hline $\mathrm{J} 0030+0451$ & 17.00 & 16.00 & $\begin{array}{ll}\text { hmvd18 } \\
\text { hmvd18 }\end{array}$ & 56304.694 & 31 & $6 / 1024$ & 4.33261 & 0.00001 & 2.49 & 0.03 & 1.32 & $\begin{array}{ll}0.07 \\
\end{array}$ & 1.17 & $\begin{array}{ll}0.08 \\
\end{array}$ & 0.332 & 0.026 & $<0.1489$ \\
\hline J0214+5222 & * & * & * & 56646.791 & 41 & $8 / 1024$ & 22.03610 & 0.00010 & -15.93 & 0.03 & 0.50 & 0.07 & -16.44 & 0.07 & -0.919 & 0.005 & $<0.0004$ \\
\hline $\mathrm{J} 0218+4232$ & * & * & * & 56473.304 & 19 & $12 / 256$ & 61.23890 & 0.00030 & -59.81 & 0.03 & 1.60 & 0.07 & -61.40 & 0.08 & -1.235 & 0.002 & $<0.0757$ \\
\hline$J 0407+1607$ & * & * & * & 56790.515 & 30 & $13 / 1024$ & 35.61091 & 0.00017 & 6.31 & 0.06 & 3.78 & 0.11 & 2.53 & 0.13 & 0.087 & 0.005 & $<0.2229$ \\
\hline J0621+1002 & 28.80 & 6.80 & hmvd18 & 56289.023 & 0 & $12 / 128$ & 36.53490 & 0.00810 & 53.91 & 0.11 & 0.7 & 0.07 & 53.20 & 0.13 & 1.794 & 0.005 & 0.0000 \\
\hline $\mathrm{J} 0636+5129$ & * & * & * & 56648.046 & 10 & $6 / 128$ & 11.10647 & 0.00021 & -1.72 & 0.08 & 0.41 & 0.06 & -2.14 & 0.10 & -0.237 & 0.013 & $<0.0005$ \\
\hline $\mathrm{J} 0645+5158$ & * & * & * & 56322.009 & 19 & $6 / 1024$ & 18.24846 & 0.00015 & -1.58 & 0.08 & 0.39 & 0.07 & -1.97 & 0.11 & -0.133 & 0.009 & $<0.0005$ \\
\hline $\mathrm{J} 1012+5307$ & 2.98 & 0.06 & nsk +15 & 56289.149 & 32 & $1 / 1024$ & 9.02436 & 0.00008 & 3.38 & 0.02 & 0.39 & 0.06 & 2.98 & 0.06 & 0.407 & 0.010 & $<0.1889$ \\
\hline $\mathrm{J} 1022+1001$ & 1.39 & 0.05 & nsk +15 & 56296.126 & 49 & $3 / 1024$ & 10.25327 & 0.00016 & 2.09 & 0.02 & 0.90 & 0.05 & 1.19 & 0.05 & 0.143 & 0.007 & $<0.2638$ \\
\hline $\mathrm{J} 1024-0719$ & -2.40 & 0.20 & $\mathrm{dhm}+15$ & 56280.16111 & 10 & $25 / 512$ & 6.48445 & 0.00025 & -1.89 & 0.06 & 1.06 & 0.07 & -2.95 & 0.09 & -0.561 & 0.022 & $<0.0005$ \\
\hline В $1257+12$ & 7.91 & 0.06 & nsk +15 & 56296.23 & 44 & $4 / 1024$ & 10.15405 & 0.00011 & 8.55 & 0.02 & 0.77 & 0.07 & 7.78 & 0.07 & 0.944 & 0.011 & $<0.2084$ \\
\hline $\mathrm{J} 1640+2224$ & 24.60 & 9.40 & hmvd18 & 56289.368 & 15 & $6 / 512$ & 18.42766 & 0.00001 & 24.22 & 0.03 & 1.41 & 0.07 & 22.81 & 0.07 & 1.525 & 0.006 & $<0.1926$ \\
\hline J1744-1134 & 2.20 & 0.20 & $\mathrm{dhm}+15$ & 56293.44 & 15 & $23 / 512$ & 3.13815 & 0.00008 & 4.22 & 0.07 & 2.63 & 0.08 & 1.59 & 0.11 & 0.625 & 0.052 & $<0.0005$ \\
\hline $\mathrm{J} 1810+1744$ & 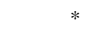 & 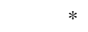 & & 56293.456 & 65 & $24 / 512$ & 39.66000 & 0.00020 & 90.15 & 0.07 & 1. & 0.06 & 88.53 & 0.10 & 2.750 & 0.004 & $<0.0004$ \\
\hline $\mathrm{J} 1923+2515$ & 10.80 & 3.80 & $1 \mathrm{br}+13$ & 56318.472 & 12 & $51 / 512$ & 18.85567 & 0.00012 & 15.44 & 0.10 & 3.1 & 0.09 & 12.34 & 0.13 & 0.807 & 0.011 & 0.0000 \\
\hline B $1937+21$ & 8.30 & 0.10 & $\mathrm{dhm}+15$ & 56434.134 & 25 & $1 / 256$ & 71.02373 & 0.00005 & 9.45 & 0.02 & 1.72 & 0.06 & 7.73 & 0.06 & 0.134 & 0.001 & $<0.2463$ \\
\hline $\mathrm{J} 2043+1711$ & (1) & 0.10 & * & 56311.522 & 14 & $3 / 256$ & 20.71232 & 0.00019 & $\begin{array}{r}-70.94 \\
-1.40\end{array}$ & 0.03 & 2.09 & 0.07 & $\begin{array}{l}-73.04 \\
-\end{array}$ & 0.08 & $\begin{array}{r}-.344 \\
\end{array}$ & 0.006 & $<0.1028$ \\
\hline $\mathrm{J} 2214+3000$ & * & * & * & 56457.159 & 7 & $13 / 128$ & 22.54310 & 0.00420 & -43.13 & 0.11 & 1.79 & 0.08 & -44.91 & 0.13 & -2.455 & 0.009 & $<0.0005$ \\
\hline $\mathrm{J} 2317+1439$ & -4.00 & 3.30 & hmvd18 & 56304.635 & 40 & $13 / 256$ & 21.89876 & 0.00026 & -8.40 & 0.01 & 1.53 & 0.07 & -9.93 & 0.07 & -0.559 & 0.005 & $<0.1760$ \\
\hline
\end{tabular}




\section{C. Sobey et al.}

\section{APPENDIX B: FDF SUMMARY}

Here, we present some additional results from analysis of the FDFs output by the RM synthesis method.

Figure B1 shows examples of some of the statistics output from testing the numbers of pulse phase bins used to output the Stokes parameters for the RM synthesis step. This includes the RM measured from the FDF, the $\mathrm{S} / \mathrm{N}$ in the $\mathrm{FDF}\left(\mathrm{S} / \mathrm{N}_{F}\right)$, and the ratio between the peak signal associated with the pulsar and the peak due to the instrumental polarisation in the FDF (PSR/Inst). Figure B1 provides an example of two pulsars: one with with relatively low S/N (PSR B0045+33); and one with high S/N (PSR B0329+54). Table B1 summarises the main results from the tests. For PSR B0045+33, the $\mathrm{RM}$ with the highest $\mathrm{S} / \mathrm{N}_{F}$ was used for the final results presented in this work (6 bins). Cumulative bin numbers $4-8$ would also be suitable, based on $\mathrm{S} / \mathrm{N}_{F} \geqslant 8$ and PSR/Inst $>1$, and these measured RMs agree within the uncertainties. For PSR B0329+54, the RM value for the final catalogue was chosen based on a compromise between relatively high $\mathrm{S} / \mathrm{N}$ and PSR/Inst (5 bins). However, due to the high $\mathrm{S} / \mathrm{N}$ and PSR/Inst for the pulsar, we see little variation between the measured RMs, which are all in excellent agreement within the uncertainties. Table B1 shows that for pulsars with lower $\mathrm{S} / \mathrm{N}$, the standard deviation for the RMs measured using different ranges of pulse phase bins is larger than for the higher $\mathrm{S} / \mathrm{N}$ case. In the low $\mathrm{S} / \mathrm{N}$ case, the standard deviation is approximately equal to the formal errors output from the RM measurement in the FDF. Due to the larger variation identified in these cases, the uncertainties from the RM measurements for pulsars with $4<\mathrm{S} / \mathrm{N}_{\mathrm{F}} \leqslant 8$ were doubled. This increased the uncertainties for 37 pulsars in the sample, but nonetheless, the uncertainties remain relatively small the maximum value is $0.13 \mathrm{rad} \mathrm{m}^{-2}$. The standard deviations for the instrumental signals in both low and high $\mathrm{S} / \mathrm{N}$ cases are identical.

Figure B2 shows the position of the instrumental peak $\left(\phi_{\text {inst }}\right)$ measured from the FDFs where a significant RM was detected. The median value is $0.04 \mathrm{rad} \mathrm{m}^{-2}$, and the standard deviation is $0.25 \mathrm{rad}$ $\mathrm{m}^{-2}$ (32 per cent of $\delta \phi$ ). The histogram and statistics show that the instrumental peak in the FDFs does not deviate greatly from $0 \mathrm{rad}$ $\mathrm{m}^{-2}$. This is generally expected since instrumental polarisation is not likely to vary with observing wavelength squared.

Figure B3 presents the FDFs obtained for each pulsar summarised in Table A1, highlighting the $\mathrm{RM}_{\mathrm{obs}}$ measured. 

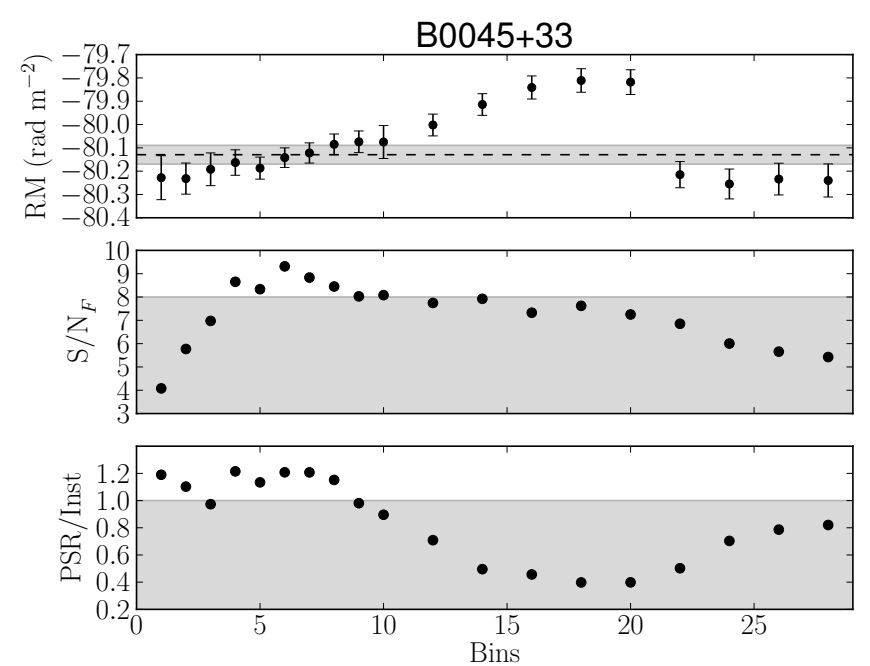

B0329+54
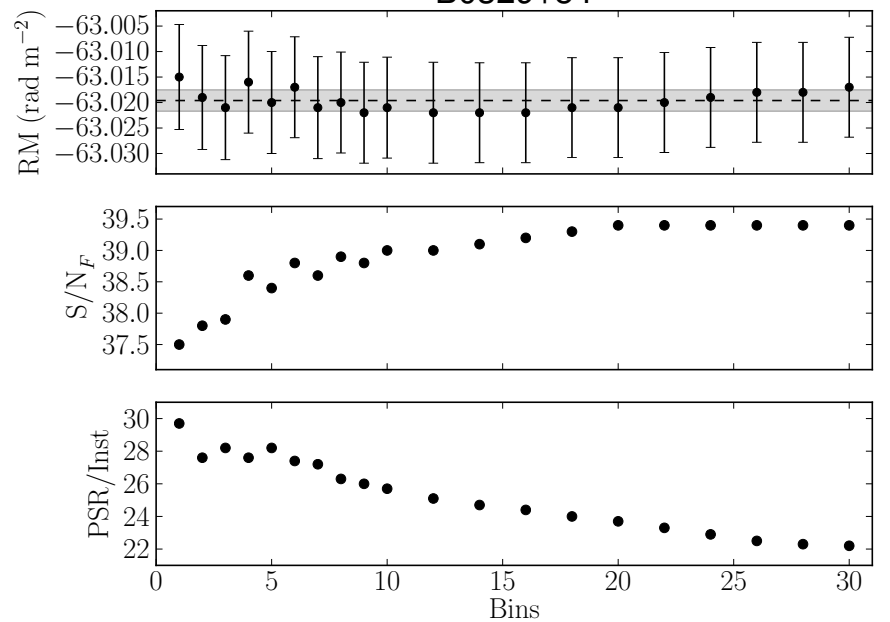

Figure B1. FDF statistics as a function of increasing numbers of pulse profile bins used to obtain the Stokes parameters from the average pulse profiles of PSR B0045+33 (left) and PSR B0329+54 (right). Top panel: measured RMs and formal uncertainties. The weighted mean RM for all measurements with S/N $\mathrm{N}_{F}>8$ is shown by the dashed line, and the weighted mean \pm the standard deviation of the measurements is shaded in grey. Middle panel: measured S/N of the signal associated with the pulsar in the FDFs, $\mathrm{S} / \mathrm{N}_{F}<8$ is shown by the shaded region. Lower panel: the ratio between the intensity of the signal associated with the pulsar and the intensity of the instrumental polarisation near $0 \mathrm{rad} \mathrm{m}^{-2}$ in the FDF (PSR/Inst). The region where PSR/Inst $<1$ shaded.

Table B1. Summary of RM statistics (in units of $\mathrm{rad} \mathrm{m}^{-2}$ ) from the Stokes parameters output using a range in pulse profile bin numbers, shown in Figure B1. For PSR B0045+33, the RM weighted mean and standard deviation were calculated using values with $\mathrm{S} / \mathrm{N}>8$.

\begin{tabular}{lrr}
\hline \hline Parameter & PSR B0045+33 & PSR B0329+54 \\
\hline Pulsar RM $\left(\mathrm{RM}_{\text {obs }}\right)$ weighted mean & -80.13 & -63.020 \\
Pulsar RM standard deviation & 0.04 & 0.002 \\
Instrumental $\left(\phi_{\text {inst }}\right)$ mean & -0.07 & -0.33 \\
Instrumental standard deviation & 0.02 & 0.02 \\
\hline
\end{tabular}

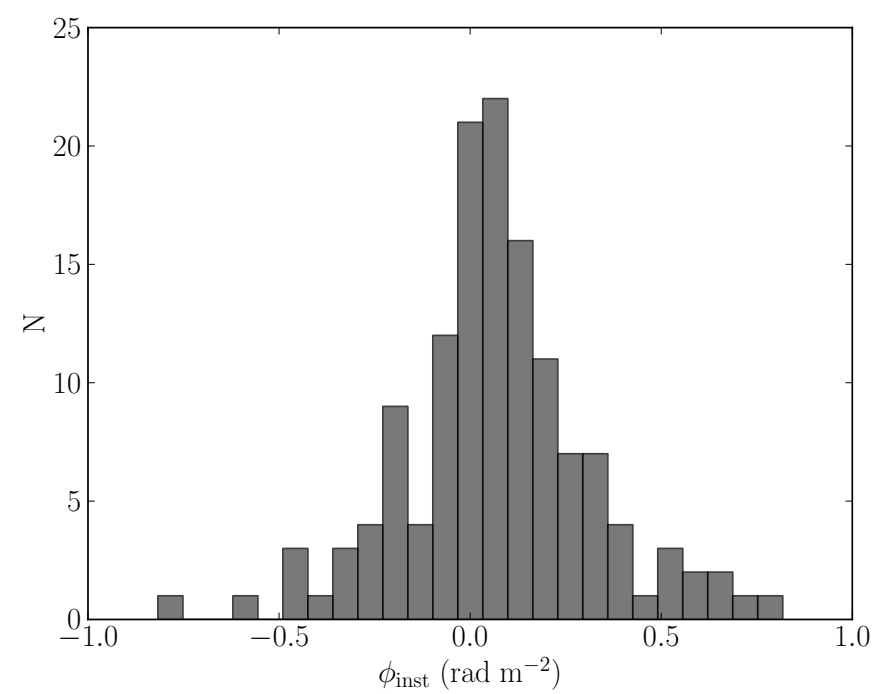

Figure B2. A histogram showing the positions of the instrumental peak measured in the FDFs $\left(\phi_{\text {inst }}\right)$ determined for all pulsars for which we obtained an RM measurement.

This paper has been typeset from a $\mathrm{TE}_{\mathrm{E}} / \mathrm{L} \mathrm{A} \mathrm{T} \mathrm{X}$ file prepared by the author. 

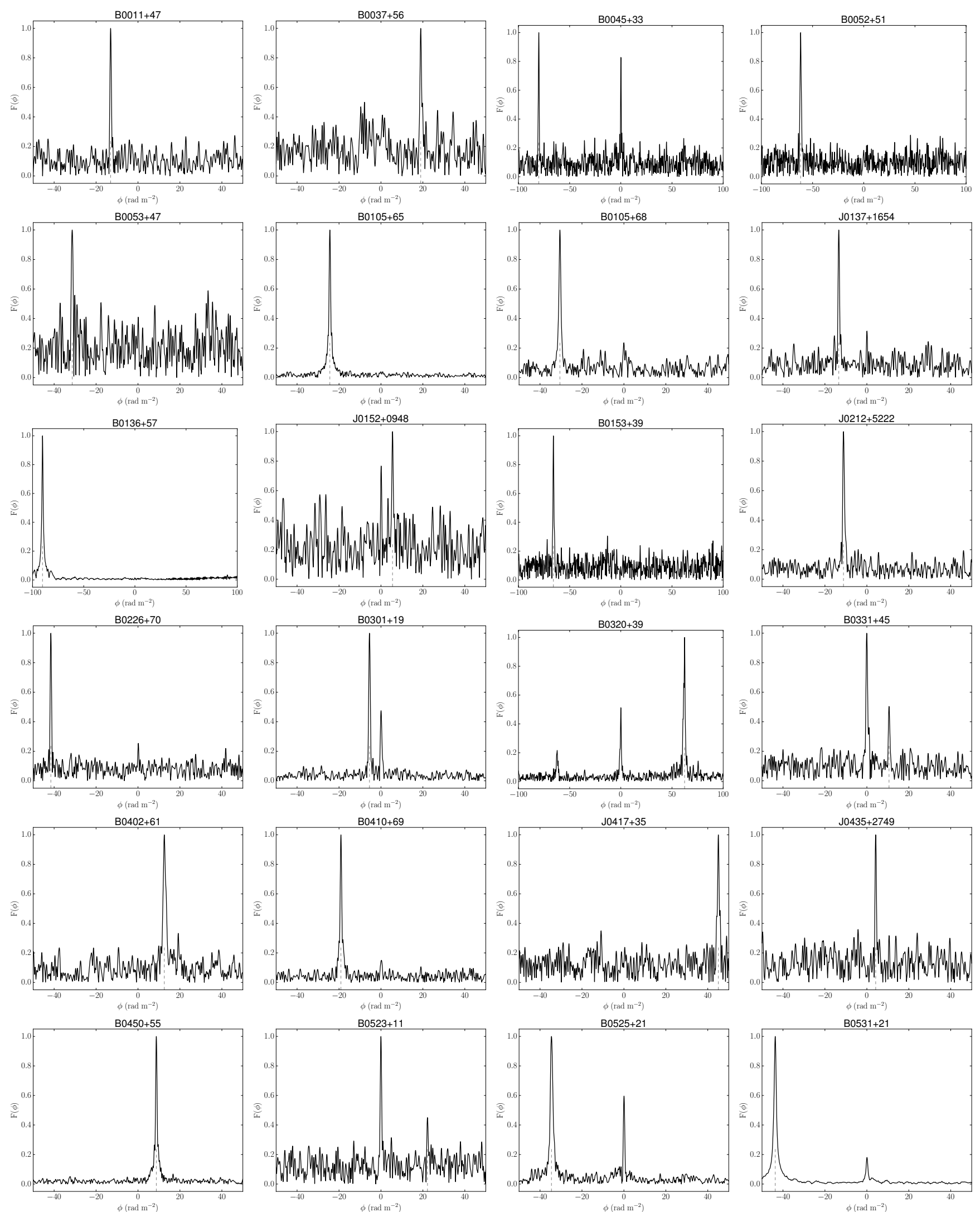

Figure B3. Faraday spectra (modulus values) for the pulsars towards which we measured an RM, ordered as in Table A1. The grey dashed line indicates the position of the peak in Faraday space, $\mathrm{RM}_{\mathrm{obs}}$, prior to any subtraction of the ionospheric RM. The $\mathrm{x}$-axes show the Faraday depth between \pm 50 , \pm 100 , \pm 150 , or $\pm 200 \mathrm{rad} \mathrm{m}^{-2}$, depending on the measured RM towards each pulsar. The y-axes show the intensity of linearly-polarised emission in arbitrary units (normalised) because the data are not flux or polarisation calibrated. Peaks near $0 \mathrm{rad} \mathrm{m}^{-2}$ are due to instrumental polarisation, and smaller symmetric peaks about 0 rad $\mathrm{m}^{-2}$ are due to polarisation leakage. 

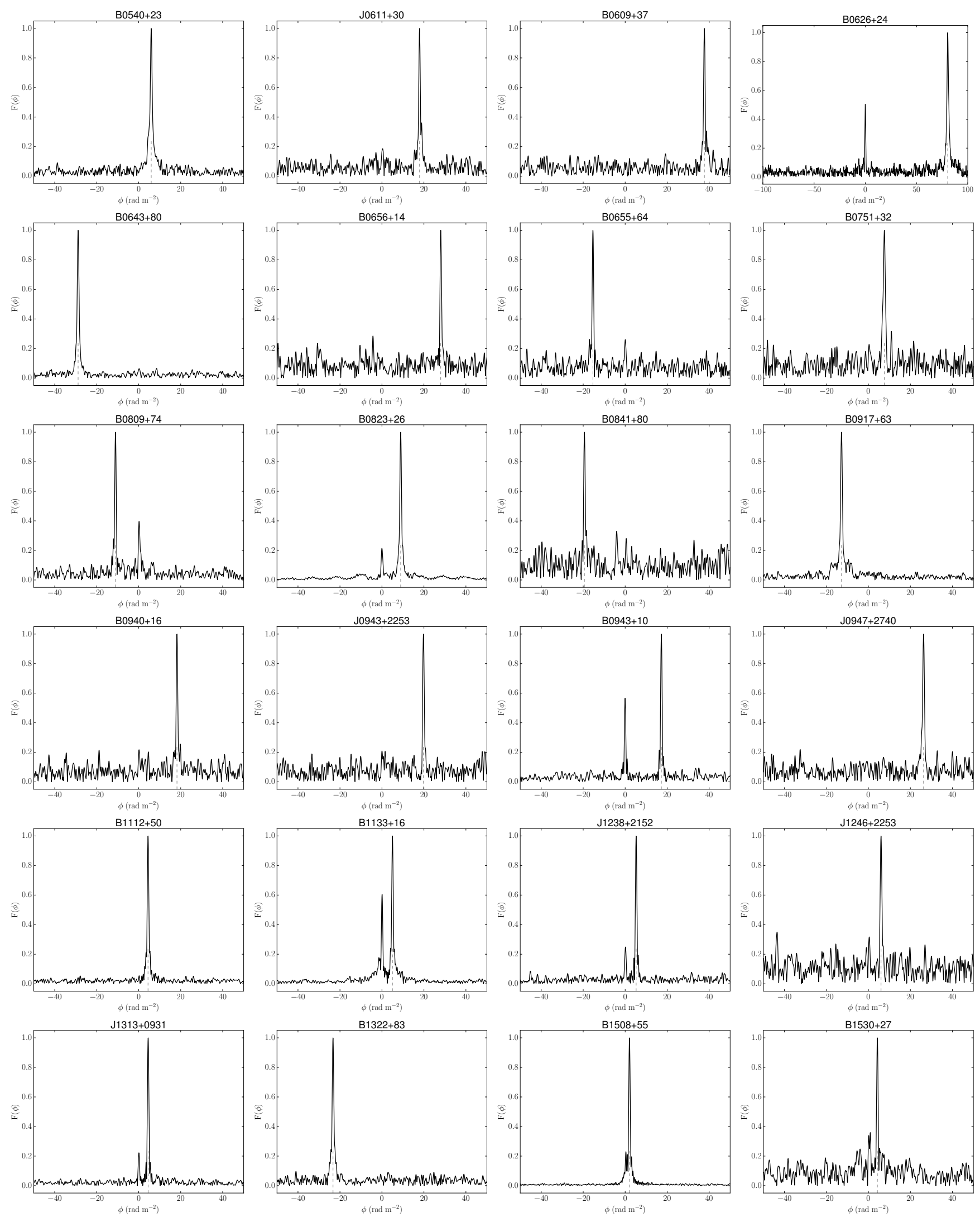

Figure B4. Continued from the previous page. 
C. Sobey et al.
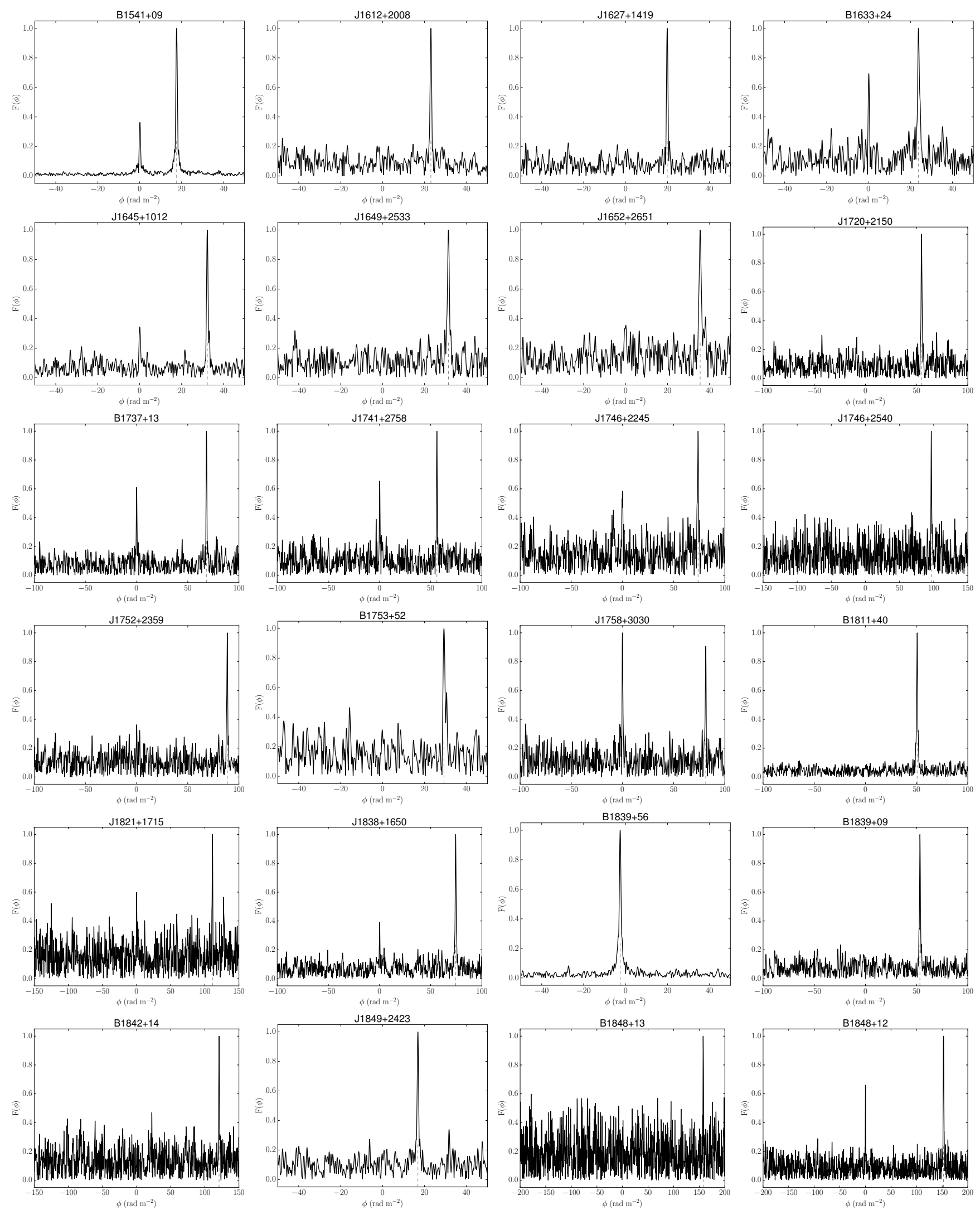

Figure B5. Continued from the previous page. 

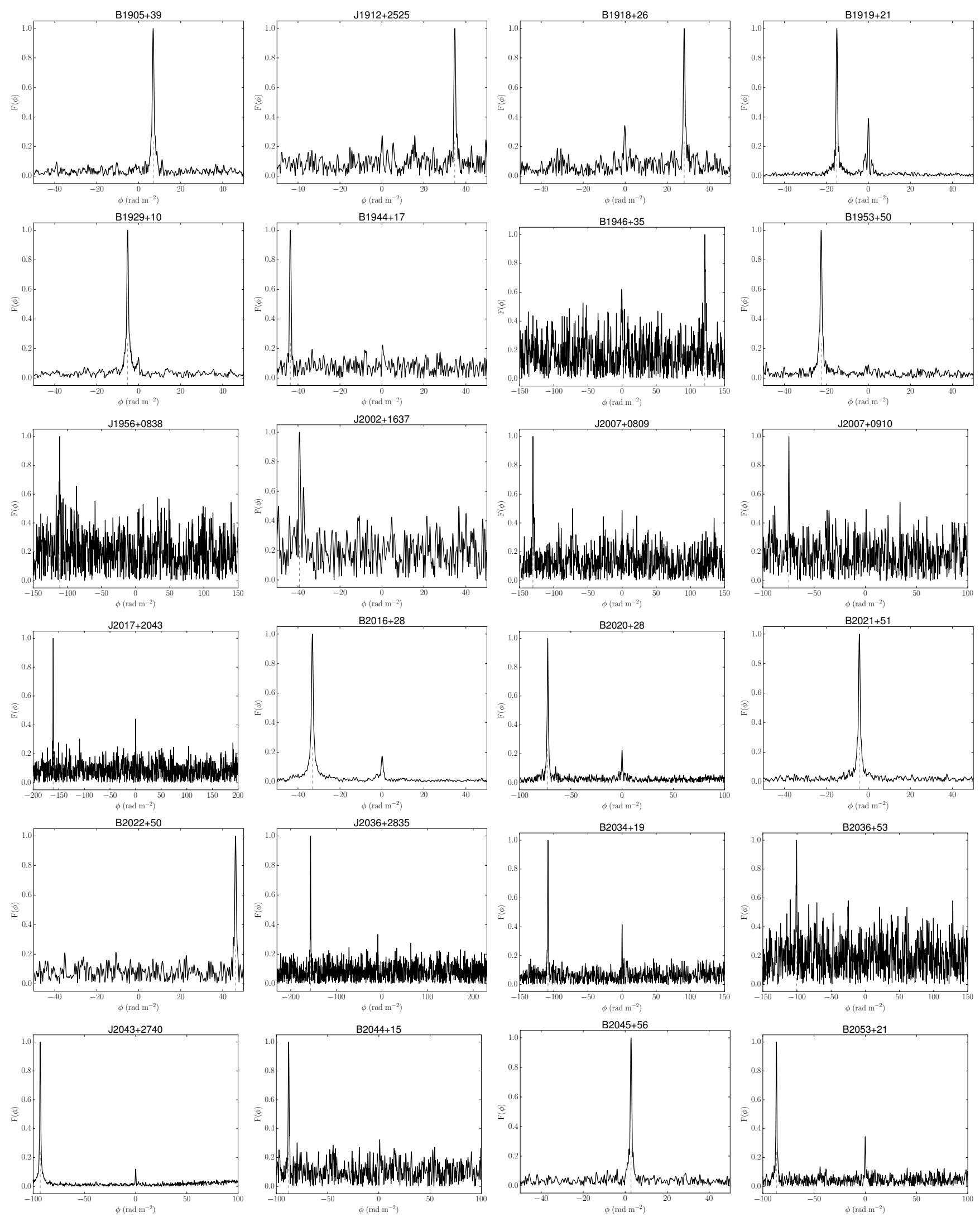

Figure B6. Continued from the previous page. 

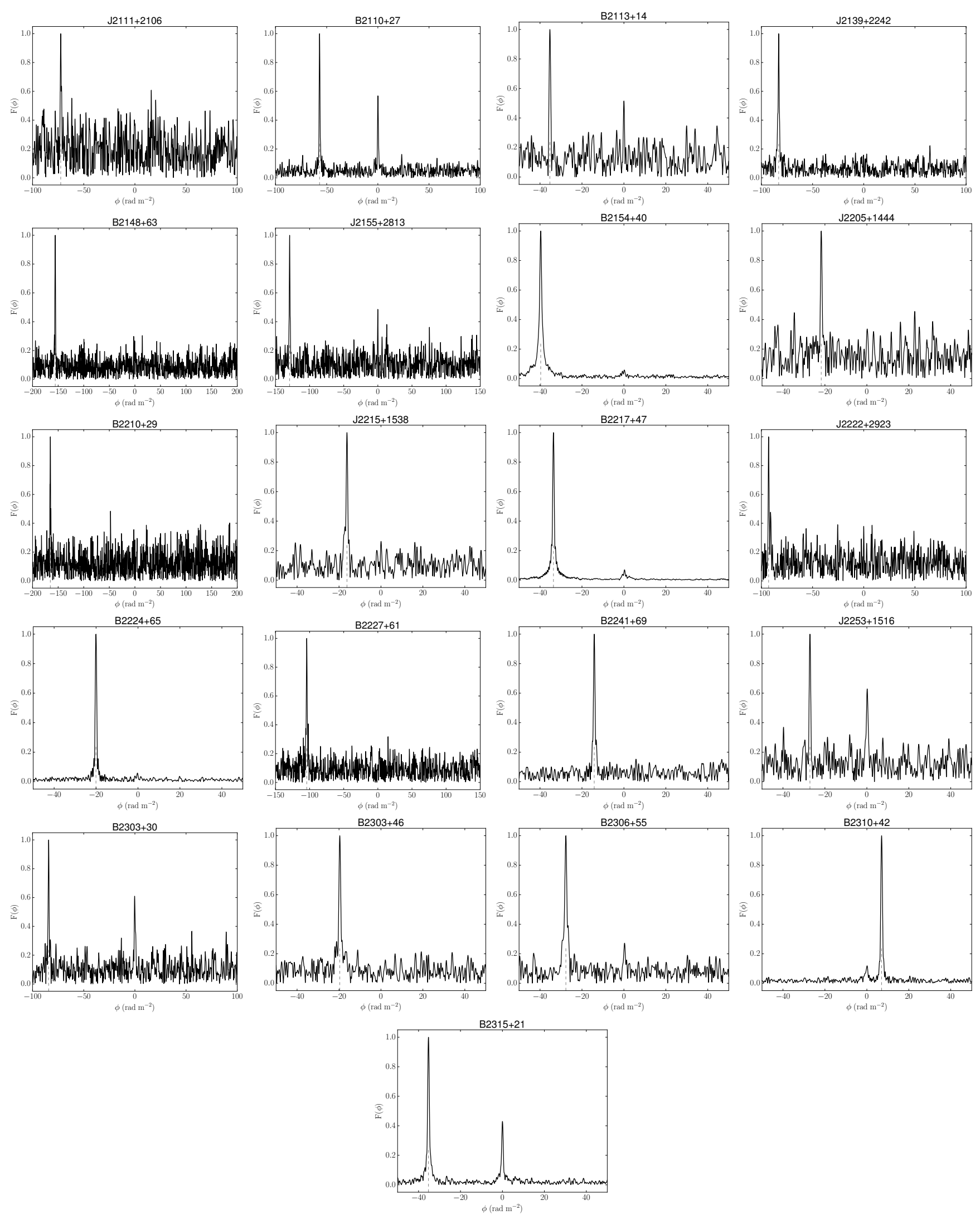

Figure B7. Continued from the previous page. 

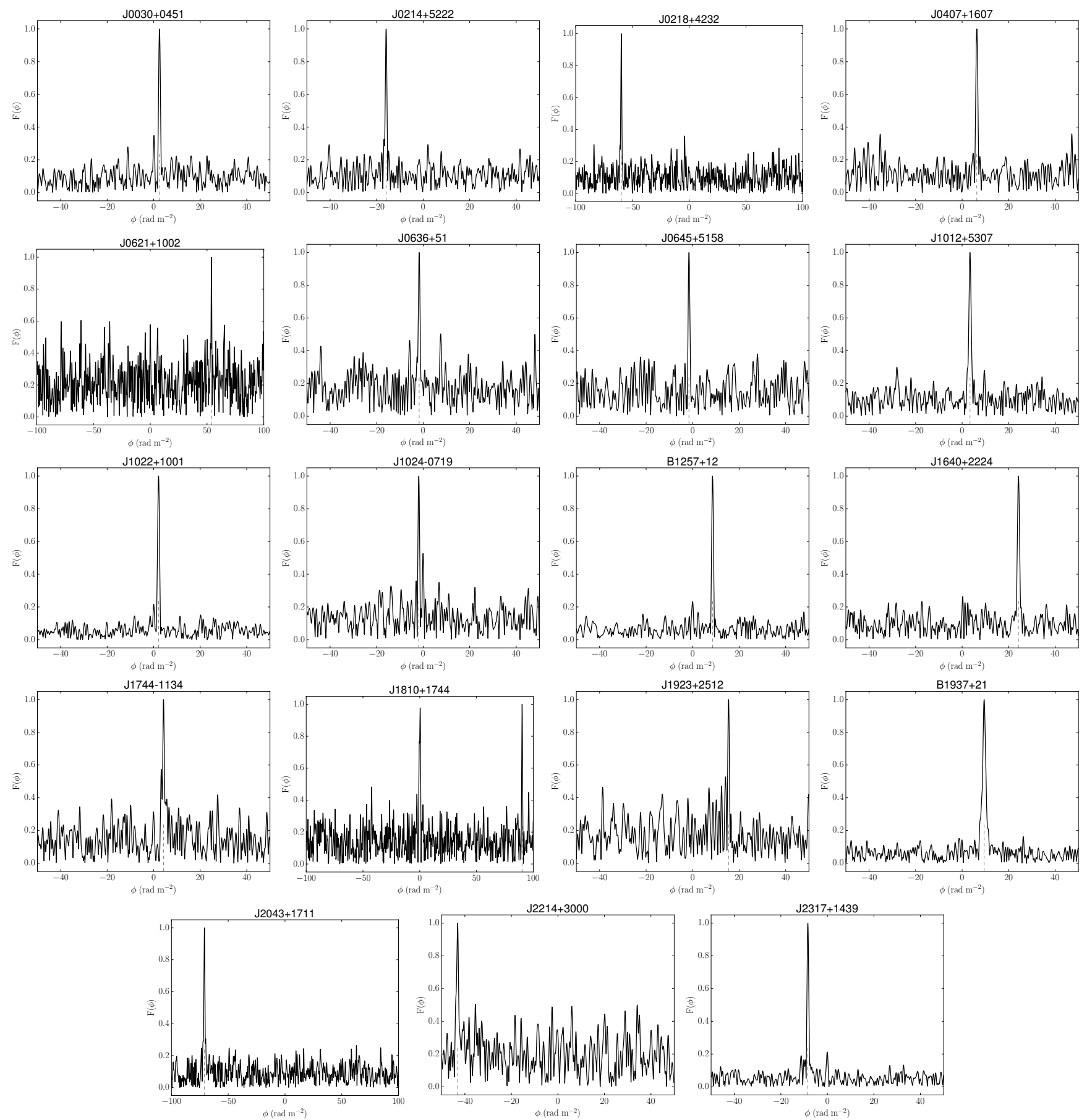

Figure B8. Continued from the previous page. 